\title{
WestVirginiaUniversity
}

THE RESEARCH REPOSITORY @ WVU

Graduate Theses, Dissertations, and Problem Reports

2021

\section{Transform Based Approaches for the Detection of Astrophysical Signals}

Marwan Mahfud Alkhweldi
malkhwel@mix.wvu.edu

Follow this and additional works at: https://researchrepository.wvu.edu/etd

Part of the Electromagnetics and Photonics Commons, and the Instrumentation Commons

\section{Recommended Citation}

Alkhweldi, Marwan Mahfud, "Transform Based Approaches for the Detection of Astrophysical Signals" (2021). Graduate Theses, Dissertations, and Problem Reports. 8302.

https://researchrepository.wvu.edu/etd/8302

This Dissertation is protected by copyright and/or related rights. It has been brought to you by the The Research Repository @ WVU with permission from the rights-holder(s). You are free to use this Dissertation in any way that is permitted by the copyright and related rights legislation that applies to your use. For other uses you must obtain permission from the rights-holder(s) directly, unless additional rights are indicated by a Creative Commons license in the record and/ or on the work itself. This Dissertation has been accepted for inclusion in WVU Graduate Theses, Dissertations, and Problem Reports collection by an authorized administrator of The Research Repository @ WVU.

For more information, please contact researchrepository@mail.wvu.edu. 


\title{
Transform Based Approaches for the Detection of Astrophysical Signals
}

\author{
Marwan Alkhweldi \\ Dissertation submitted to the \\ College of Engineering and Mineral Resources \\ at West Virginia University \\ in partial fulfillment of the requirements \\ for the degree of \\ Ph.D. \\ in \\ Electrical Engineering \\ Natalia A. Schmid, D.Sc., Chair \\ Kevin Bandura, Ph.D. \\ $\mathrm{Yu}$ Gu, Ph.D. \\ Matthew C. Valenti, Ph.D. \\ Brian D. Woerner, Ph.D. \\ Lane Department of Computer Science and Electrical Engineering \\ Morgantown, West Virginia \\ 2021
}

Keywords: Radio Astronomy, Fast Radio Bursts, Rotating Radio Transients, Pulsar, Dispersion Measure, Filter Bank Data, Spectrogram, Radon Transform, Detection, Estimation, Phased Array Feeds, Inferential Statistics, Generalized Likelihood Ratio Test 


\begin{abstract}
Transform Based Approaches for the Detection of Astrophysical Signals
\end{abstract}

Marwan Alkhweldi

Development of new algorithms for the detection of isolated astrophysical pulses is of interest to radio astronomers. Both Fast Radio Bursts (FRBs) and several Rotating Radio Transients (RRATs) were detected through the application of a single pulse search algorithm. The conventional approach to detect astronomical pulses requires an exhaustive search for the correct dispersion measure. Its accelerated versions involve signal processing in Fourier transform space.

In this dissertation, we present several new transform-based approaches for the detection and analysis of astrophysical signals with the latest being the most effective and advanced of all. It is implemented in several steps. First, a spectrogram of a dispersed astrophysical pulse is linearized in observing frequency followed by application of the Radon transform. The result of the transformation is displayed as a two-dimensional function. Next, the function is smoothed using a spatial low-pass filter. Finally, the maximum of the function above 90-degree angle is compared to the maximum of the standard deviation of the noise below 90-degree angle and a decision in favor of an astrophysical pulse present or absent is made. Once pulse is detected, its Dispersion Measure (DM) is estimated by means of a basic equation relating the slope of the linearized dispersed pulse and the DM value. Performance of the algorithm is analyzed by applying it to a set of simulated Fast Radio Bursts, experimental data of Masui pulse and experimental data of seven Rotating Radio Transients. The detection algorithm demonstrates results comparable to and above those by the conventional pulse detection algorithm. 


\section{Contents}

\begin{tabular}{ll}
\hline List of Figures & v \\
\hline
\end{tabular}

\begin{tabular}{lll}
\hline & Introduction & 1
\end{tabular}

1.1 Introduction . . . . . . . . . . . . . . . . . . . . . . . 1

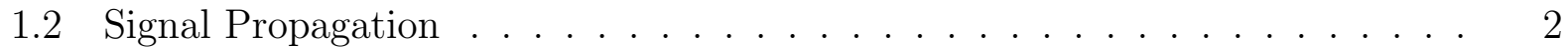

1.2 .1 Dispersion and Scattering . . . . . . . . . . . . . . . . . . . . . . 4

1.2 .2 Noise and Radio Frequency Interference . . . . . . . . . . . . . . . . 4

1.2 .3 Spectrogram . . . . . . . . . . . . . . . . . . . 5

1.3 Search algorithms . . . . . . . . . . . . . . . . . . . . . 6

1.4 Dissertation Outline . . . . . . . . . . . . . . . . . . . . . . . . . . . . . 9

2 Detection of FRBs based on SFT and Radon Transform 10

2.1 Introduction . . . . . . . . . . . . . . . . . . . . 10

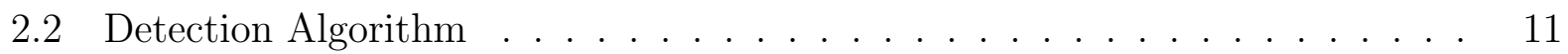

2.2 .1 Radon transform . . . . . . . . . . . . . . . . . . . . . 11

2.2 .2 One dimensional Fourier transform . . . . . . . . . . . . . . . . . . . 14

2.2 .3 Cumulative magnitude Fourier transform . . . . . . . . . . . . . . . . 14

2.2 .4 Decision rule . . . . . . . . . . . . . . . . . . . . . . . . . . . . . . . . . . . . . .

2.3 Estimation of DM value . . . . . . . . . . . . . . . . . . . . . . . . 15

2.4 Bounds on DM Value . . . . . . . . . . . . . . . . . . . . . . . . . . . 16

2.5 Performance Analysis . . . . . . . . . . . . . . . . . . . . . . . . 17

2.5 .1 Numerical Evaluation based on Simulated Data . . . . . . . . . . . . 18

2.5 .2 Numerical Evaluation based on Practical Data . . . . . . . . . . . . . 21

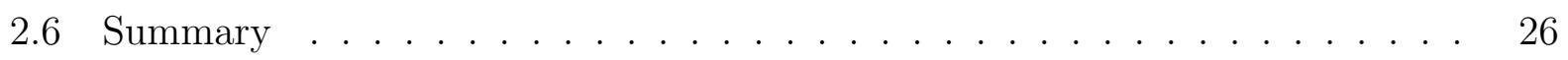

3 Detection of Periodic Astrophysical Pulses 28

3.1 Introduction . . . . . . . . . . . . . . . . . . . . . . . . . . . . . . . . . 28

3.2 SFT Signature of a Periodic Signal . . . . . . . . . . . . . . . . . . . . . . 28

3.3 Estimation of Rotation . . . . . . . . . . . . . . . . . . . . . . . . . . 31

3.4 DM Estimation . . . . . . . . . . . . . . . . . . . . . . . . . . . . . . 32

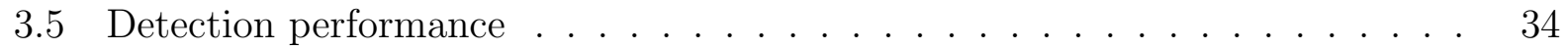

3.6 Summary . . . . . . . . . . . . . . . . . . . . . . . . . . . 35 
\begin{tabular}{|lll}
4 & The Effect of Linearized Spectrogram & 36
\end{tabular}

4.1 Introduction . . . . . . . . . . . . . . . . . . . . 36

4.2 Detection Algorithm . . . . . . . . . . . . . . . . . . . . . . 37

4.2 .1 Linearization . . . . . . . . . . . . . . . . . . . . . . . . . . . . . . . . . . . . . . . . . .

4.2 .2 Radon transform . . . . . . . . . . . . . . . . . . . . . . 40

4.2 .3 Spatial Smoothing . . . . . . . . . . . . . . . . . . . . . . . . . 42

4.2 .4 Decision rule ... . . . . . . . . . . . . . . . . . . . 44

4.3 Estimation of DM Value . . . . . . . . . . . . . . . . . . . . . . . . . . 45

4.4 Detection of Periodic Astrophysical Pulses . . . . . . . . . . . . . . . . . . . . . 46

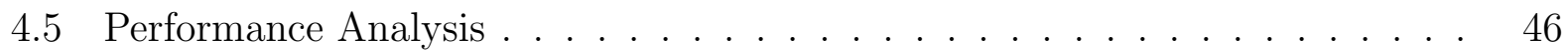

4.5 .1 Numerical Evaluation based on Simulated Data . . . . . . . . . . . . 48

4.5 .2 Numerical evaluation based on experimental data . . . . . . . . . . . . 56

4.6 Summary . . . . . . . . . . . . . . . . . . . . . . . . . 62

5 SFT for the Detection of Isolated Astronomical Pulses 64

5.1 Introduction . . . . . . . . . . . . . . . . . . . . . 64

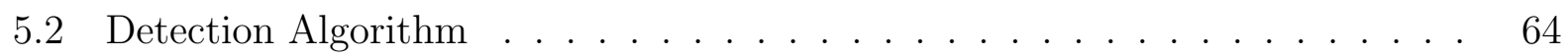

5.2 .1 Linearization . . . . . . . . . . . . . . . . . . 64

$5.2 .2 \quad$ Spatial Fourier transform (SFT) . . . . . . . . . . . . . . . . 65

5.2 .3 Decision rule . . . . . . . . . . . . . . . . . . . . 67

5.2 .4 Estimation of Parameters . . . . . . . . . . . . . . . . . . 68

5.3 Performance Analysis $\ldots \ldots \ldots$. . . . . . . . . . . . . . . . . . . . . . . . 69

5.3 .1 Numerical Evaluation based on Simulated Data . . . . . . . . . . . . 70

5.3 .2 Numerical evaluation based on experimental data . . . . . . . . . . . 72

5.4 Summary . . . . . . . . . . . . . . . . . . . . 76

6 Detection in Radio Astronomy Dense Aperture Arrays 79

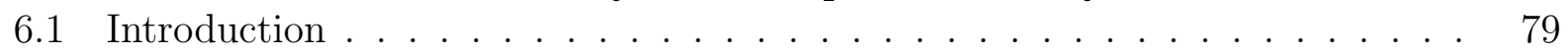

6.2 Assumptions and Models . . . . . . . . . . . . . . . . . . . . . . . 81

6.3 Inferential Statistics $\ldots \ldots \ldots$. . . . . . . . . . . . . . . . . . . . 82

6.3 .1 Detection Using a Deterministic Source Signal Model . . . . . . . . . 82

6.3 .2 Detection Using a Random Source Signal Model . . . . . . . . . . . . 84

6.4 Numerical Evaluation . . . . . . . . . . . . . . . . . . . . . . . . . 87

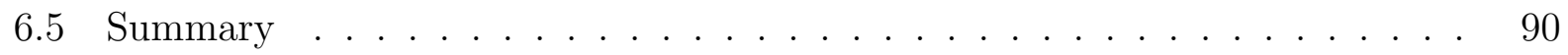

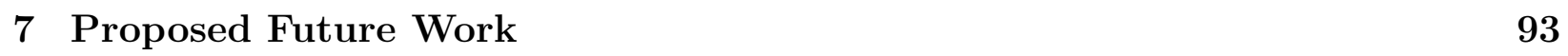

$7.1 \quad$ Stochastic description of raw voltages . . . . . . . . . . . . . . . . . . . 93

7.2 Detection rule . . . . . . . . . . . . . . . . . . . . . . . . . 94

\begin{tabular}{ll}
\hline A Related Publications & 96
\end{tabular}

\begin{tabular}{lr}
\hline References & 97
\end{tabular} 


\section{List of Figures}

$1.1 \quad$ Signal Propagation. . . . . . . . . . . . . . . . . . . . . . . . . 3

1.2 Examples of RFI and astronomical signals $|1| \ldots \ldots \ldots$. . . . . . . . . . 5

1.3 Main steps of the conventional detection approach applied to Lorimer Burst and Noise+RFI only filter bank data. . . . . . . . . . . . . . . . 7

$1.4 \quad$ Main steps of the conventional detection approach in application to a spectrogram of the millisecond pulsar B1937+21. . . . . . . . . . . . . 8

$2.1 \quad$ A block diagram of the proposed detection approach. . . . . . . . . . . . . 11

2.2 The result of applying the Radon transform to the Lorimer burst at three different orientations $\theta=30^{\circ}, \theta=60^{\circ}$, and $\theta=76^{\circ} . \ldots \ldots \ldots 12$

2.3 Filter bank data in (a) and (b) and their step-wise transformations. . . . . . 13

$2.4 \quad$ A sensitivity plot displaying the dependence of the estimated SNR values using the conventional algorithm versus the width of pulses. The input SNR

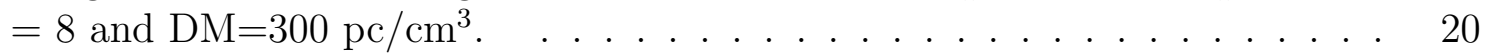

2.5 A sensitivity plot displaying the dependence of the estimated SNR values using the conventional algorithm versus the width of pulses. The input SNR

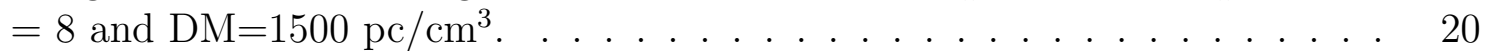

2.6 Shown are interval estimates of the DM value of the FRBs listed in Table 2.1 . Each panel presents a plot of the estimated DM value versus the realization number, the true DM value and the DM values due to the bounds in Equations

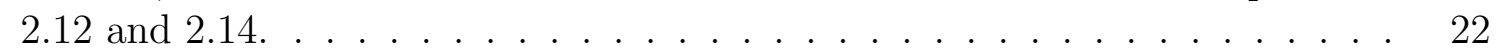

$2.7 \quad$ Masui pulse data in (a) and its step-wise transformations in (b) through (d). 23

2.8 Estimated DM values for the detected pulses of four RRAT series. Bounds on the DM value of the new candidate pulses are marked with red triangles. . . 25

3.1 Shown are an isolated pulse and a periodic signal in filter bank and SFT formats. 29

3.2 SFT signature of a chunk of PSR B1937+21 integrated over $v$ in the third and forth quadrants of the SFT space. . . . . . . . . . . . 30

$3.3 \quad$ Signature of PSR B1937+21 in the SFT space. . . . . . . . . . . . . . 32

3.4 Estimation of DM from 100 randomly selected windows of PSR B1937+21. . 34

3.5 Probability of detection as a function of observation time window. . . . . . 35

$4.1 \quad$ A block diagram of the proposed detection approach. . . . . . . . . . . . . . 37

4.2 Shown on the same plot are a dispersed pulse as a function of time and frequency and its linearized version. $\ldots \ldots \ldots \ldots . \ldots . \ldots 38$ 
4.3 Illustration of the linearization. $\ldots \ldots \ldots$. . . . . . . . . . . . . . . . . . . . 39

4.4 The result of applying the Radon transform to the Lorimer burst at two different orientations $\theta=40^{\circ}$ and $\theta=140^{\circ}$. . . . . . . . . . . . . . . . 40

4.5 Spectrograms of the experimental data of Lorimer burst (shown in panel(a)) and of noise and RFI (shown in panel (b)) and their step wise transformations. 41

4.6 Three-dimensional surface plot of Radon image. . . . . . . . . . . . . . 43

4.7 Three-dimensional surface plot of filtered Radon image. . . . . . . . . . . . . 43

4.8 Spectrogram of a simulated millisecond pulsar shown in panel (a) and its step wise transformations. . . . . . . . . . . . . . . . . . 47

4.9 Shown are two bar plots displaying the dependence of the number of detected pulses by our and conventional algorithms versus the width of pulses. The input SNR is set to 8, the number of simulated pulses for each DM is set to 10,000 , and the value of DM is set to $300 \mathrm{pc} / \mathrm{cm}^{3}$ in the left panel and to 1500

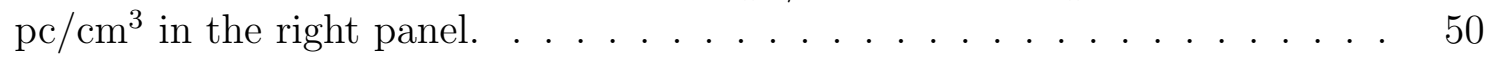

4.10 Shown are two bar plots displaying the dependence of the number of detected pulses by our and conventional algorithms versus the width of pulses. The input SNR is set to 8, the number of simulated pulses for each DM is set to 1,000 , and the value of DM is set to $300 \mathrm{pc} / \mathrm{cm}^{3}$ in the left panel and to 1500 $\mathrm{pc} / \mathrm{cm}^{3}$ in the right panel. $\ldots \ldots \ldots . \ldots . \ldots 51$

4.11 Shown are two sets of bar plots displaying the dependence of the number of detected pulses by our and conventional algorithms on the width of pulses. In this experiment one fifth of the spectrogram is corrupted by RFI. . . . . . . 52

4.12 Shown are two bar plots displaying the dependence of the number of detected pulses by our and conventional algorithms versus the width of pulses. In this example about one third of the spectrogram is corrupted by RFI. . . . . . . 53

4.13 Shown is a bar plot displaying the dependence of the number of detected pulses by our and conventional algorithms versus the width of pulses. The number of simulated pulses for each DM is set to 1,000, and the value of DM is set to $300 \mathrm{pc} / \mathrm{cm}^{3}$. . . . . . . . . . . . . . . . . . . 54

4.14 Shown are estimates of the DM value for several FRBs listed in Table 4.1 . Each panel presents a plot of the estimated DM value versus the instance number and the true DM value. . . . . . . . . . . . . . . . . . . . . . 55

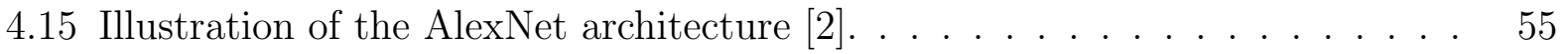

4.16 Shown are the input data to AlexNet and the performance of AlexNet associated with the input data. . . . . . . . . . . . . . . . . . . 56

4.17 Shown are two bar plots summarizing the performance of AlexNet. . . . . . 57

4.18 Masui pulse spectrogram in (a) and its step-wise transformations in (b) through

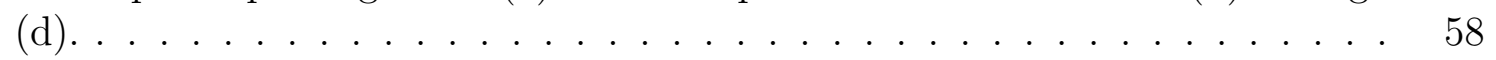

4.19 Estimated DM values for the detected pulses of four RRAT series. The new candidate pulses are marked in red. . . . . . . . . . . . . . . . . . 60

4.20 Main steps of the detection approach in application to a spectrogram of the millisecond pulsar B1937+21 real data. . . . . . . . . . . . . . . . . 61

4.21 Estimated DM values for different chunks of B1937+21. . . . . . . . . . . . . 62 
5.1 Shown are an isolated pulse and a periodic signal and their step wise trans-

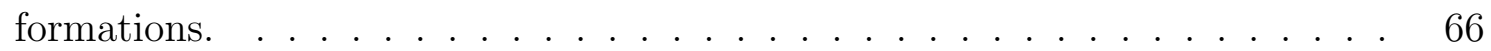

5.2 Examples of RFI signals in spectrograms and in the SFT domain. . . . . . . 68

5.3 Shown are two bar plots displaying the dependence of the number of detected pulses by our and conventional algorithms versus the width of pulses. The input SNR is set to 8, the number of simulated pulses for each DM is set to 1,000 , and the value of DM is set to $300 \mathrm{pc} / \mathrm{cm}^{3}$ in the left panel and to 1500 $\mathrm{pc} / \mathrm{cm}^{3}$ in the right panel. . . . . . . . . . . . . . . . . . . . 71

5.4 Shown are estimates of the DM value for two FRBs listed in Table 5.1 . Each panel presents a plot of the estimated DM value versus the instance number and the true DM value. . . . . . . . . . . . . . . . . 72

5.5 Spectrograms of the experimental data of Lorimer burst (shown in panel(a)) $\begin{array}{ll}\text { and of Masui pulse (shown in panel (b)) and their step wise transformations. } & 74\end{array}$

5.6 Estimated DM values for the detected pulses of two RRAT series. . . . . . . 75

5.7 Main steps of the detection approach in application to a spectrogram chunk of the millisecond pulsar B1937+21. . . . . . . . . . . . . . . . . . 77

6.1 Shown is a 20 millisecond interval of the $B 1937+21$ time series. Two red circles mark two adjacent pulses of the signal of interest. Note that some of the noise pulses have much larger amplitude than the signal pulses. . . . . . 88

6.2 Performance of the rules $g_{1}$ (left panel) and $g_{2}$ (right panel) applied to the $B 1937+21$ time series and parameterized by the number of time samples used to implement the rules. . . . . . . . . . . . . . . . . . . . . . . . . . . . . . . 89

6.3 Performance of the rules $g_{3}$ applied to the $B 1937+21$ time series and parameterized by the number of time samples used to implement the rules. . . . . 89

6.4 Performance of the rules $g_{1}$ (left panel) and $g_{2}$ (right panel) applied to the $J 1125+78$ time series and parameterized by the number of time samples used to implement the rules. . . . . . . . . . . . . . . . . . . . . 91 


\section{Chapter 1}

\section{Introduction}

\section{$1.1 \quad$ Introduction}

Development of new algorithms for the detection of astrophysical pulses is of interest to radio astronomers. This dissertation offers several new algorithms. It also analyses the performance of every developed algorithm.

Both Fast Radio Bursts (FRBs) and individual pulses of Rotating Radio Transients (RRATs) were detected through the application of a single pulse search algorithm, while pulsars are traditionally detected using periodic searches. FRBs are short-duration isolated radio bursts of extragalactic nature [3 5]. The origin of these mysterious sources is currently unclear. Some possible sources that have so far been proposed to explain FRBs include collapsing neutron stars [6], coalescing neutron-star binaries [7], evaporating black holes [8], or cosmic strings. Efficiently finding more bursts will unlock their cause and may enable the use of FRBs to probe the universe.

Pulsars, highly magnetized rotating neutron stars, have been under intense study since their discovery in late 60s [9]. They have been used to investigate a wide variety of topics, including exotic matter physics, low-frequency gravitational waves, fundamental tests of general relativity, interstellar weather, globular cluster astrophysics, extrasolar planets, and planetary physics. RRATs are a special category of pulsars, spinning magnetized neutron stars 10 17. Unlike pulsars, RRATs emit energy sporadically. RRATs were discovered through a single pulse search of the Parkes Multibeam Pulsar Survey data [18]. RRATs are 
observed as smeared isolated pulses, and are thought to be pulsars which emit much more sporadically, or with much higher pulse to pulse amplitude variability than normal pulsars.

Astrophysical pulses are broad spectrum sources that emit their radio signals over a wide bandwidth. The observed signals are dispersed and relatively weak compared to many discovered pulsars and other celestial emissions. Furthermore, the observed signals are usually corrupted by noise and some radio frequency interference (RFI).

From signal processing perspective, detecting astronomical pulses is a challenging task, especially isolated pulses. Radio astronomy data are extremely noisy, and the location of pulses in the data are not known. When a telescope such as the Green Bank Telescope [19] is used to survey the sky, gigabytes of radio astronomical data are streamed and stored on disks in a matter of minutes. Therefore searching for the pulses in real time would save not only the processing time but also the storage space.

\subsection{Signal Propagation}

In this section, we describe several concepts essential to understand the work summarized in this dissertation. Fig. 1.1 shows a block diagram of the signal propagation model used in radio astronomy. The same block diagram explains the bulk of signal processing applied to the astronomical signal after it has been received by a telescope.

Radio signals generated by astronomical sources are broadband signals (see Fig. 1.1(a), Fig. 1.1(b) and Fig. 1.1(c)). They travel through the interstellar medium (see Fig 1.1(d)) that causes signal distortions, dispersion and scattering (see Fig 1.1(f) and Fig 1.1(g)). Once the signal reaches the ground, it has other terrestrial radio signals, instrument noise and thermal background noise (see Fig 1.1(h), Fig 1.1(i), and Fig 1.1(j)) added to it. Since the bandwidth of a radio telescope is finite, it can capture only a portion of the spectrum of the broadband astronomical signal (see Fig 1.1(k) and Fig 1.1(l)). Raw signal observations by the telescope pass through different stages of amplification, filtering, and frequency conversion and at the end they are displayed in the form of a spectrogram (see Fig 1.1(n)) that are then stored on disks for offline searches. 


\subsubsection{Dispersion and Scattering}

Astronomical signals are broad band signals that are emitted over a wide bandwidth. Radio astronomical telescopes are designed to receive such signals, typically of order of hundreds of MHz. During the signal travel through the interstellar medium (an environment filled with free electrons), the signal experiences dispersion. A signal dispersion is what distinguishes astrophysical pulses from other signals present in astrophysical data. In a dispersed pulse, the lower frequency components of the pulse are delayed compared to higher frequency components. The delay time $\Delta t$ (sec.) due to dispersion at frequency $f(\mathrm{MHz})$ is

$$
\Delta t=415 \times D M \times\left(f^{-2}-f_{0}^{-2}\right)
$$

where $f_{0}$ is a reference frequency, with respect to which the delay is measured (usually it is the center frequency of a receiver),

$$
\mathrm{DM}=\int_{0}^{D} n_{e} d l \approx n_{e} \times D
$$

is the dispersion measure in $p c / \mathrm{cm}^{3}, n_{e}$ is the mean electron density in $\mathrm{cm}^{-3}$ and $D$ is the distance to signal source in $p c$. From Eqs. 1.1 and 1.2, the time delay is proportional to the density of free electrons in the interstellar medium (ISM) integrated over the distance that the pulse travels and also proportional to the reciprocal of squared frequency.

Scattering is another effect that an astrophysical pulse is experiencing while traveling through the ISM. The consequence of scattering is that low frequencies of a pulse experience greater broadening and stretching than the higher frequencies of the pulse. Signals from a farther source are more likely to experience scattering (see Fig. 1.1(g) for illustration) compared to a source located closer to Earth.

\subsubsection{Noise and Radio Frequency Interference}

Radio telescopes (the Green Bank Telescope is one of examples) are often built away from large cities to avoid Radio Frequency Interference. In spite of all efforts to lower the level of noise and avoid RFI, Radio astronomy data are usually extremely noisy, with SNR down to -50dB. Noise sources include spillover noise, sky noise, thermal noise from antenna 


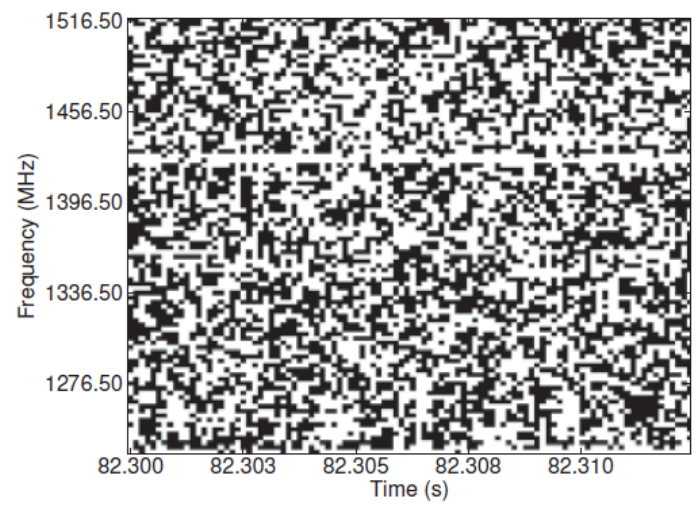

(a) Channelized RFI around $1425 \mathrm{MHz}$

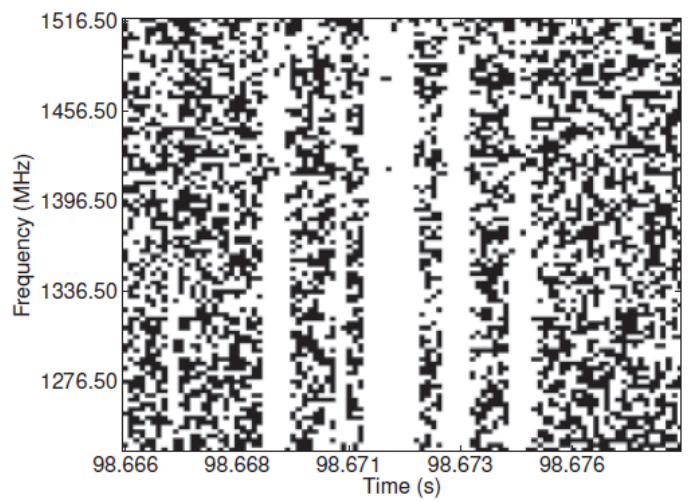

(c) An RFI event of several broadband bursts

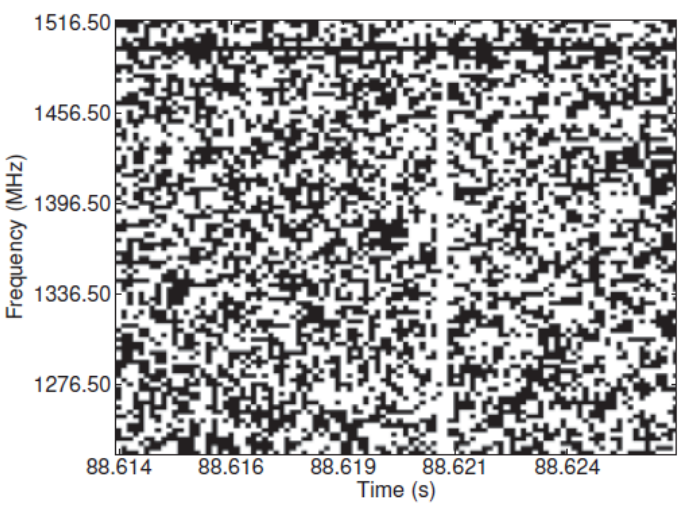

(b) Broadband, short-duration RFI

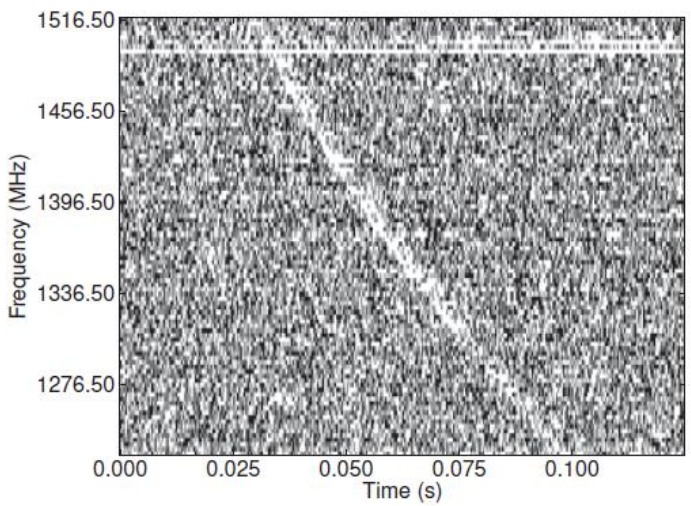

(d) A dispersed astronomical signal

Figure 1.2: Examples of RFI and astronomical signals $\mid 1$.

elements, and amplifier noise. RFI sources include nearby electrical devices (e.g., desktop computer), and communication systems (e.g., airport radar systems). RFI is characterized by a zero DM value and may be broadband at few time samples (bursty) or persistent at few frequency channels for long duration. An example of RFI in real spectogram data is shown in Fig. 1.3(a), RFI is the line at frequency around $1340 \mathrm{MHz}$. Fig. 1.2 shows more examples of RFI.

\subsubsection{Spectrogram}

The telescope observations are raw voltage signals collected as functions of time. The raw signal gets processed as it passes through the receiver and the back-end systems which converts raw telescope data into a two-dimensional array of time and frequency known in 
radio astronomy as spectrogram, filter bank data, or (t,f) plane, interchangeably.

To obtain a spectrogram from raw data, a Short-Time Fourier Transform (STFT) is applied to the data. While the window defining the STFT slides along the data, the transformed data are reassembled into columns of a spectrogram. At the end, the complex valued entries of the spectrogram are replaced by their magnitude squared values (power). Examples of a spectogram of practical astrophysical pulses are shown in Fig. 1.3(a), Fig. 1.4(a) and Fig. 1.2(d). The dispersed pulse in Fig. 1.3(a) and in Fig. 1.2(d) shows up as a quadratic curve in the $(t, f)$ plane, the dispersed pulses in Fig. 1.4(a) are invisible to the naked eye.

\subsection{Search algorithms}

A well-established conventional approach [20] to the astronomical pulse detection operates on spectrograms. Its first step is to compensate for the delay in each frequency channel (known as de-dispersion) and then to integrate the de-dispersed signal over the entire frequency range 21. This process boosts the Signal-to-Noise Ratio (SNR) of the astronomical pulse. The conventional de-dispersion followed by a matched filter detection approach involves an exhaustive search for the correct DM over a broad range of possible values. In radio astronomy, signal-to-noise ratio is defined as a ratio of the peak value of a de-dispersed and matched-filtered observed signal, to the root mean square (RMS) of filtered noise. Most of FRBs known today were discovered using the conventional approach and its optimized variants 22 26. Demonstration of the conventional approach main steps applied to experimental data is shown in Fig. 1.3 .

Traditionally new pulsars are detected by means of a periodic search [10], where after removal of RFI, data are de-dispersed at a certain DM value, integrated over frequency and then decomposed into a Fourier series. Given a sufficiently long observation time, the Fourier series decomposition leads to the detection of harmonics, which in turn allows estimation of the rotational period of a pulsar. Once the period is estimated, de-dispersed and integrated in frequency, data are folded multiple times yielding a single pulse of maximal SNR value. Fig. 1.4 shows the main steps of the periodic search as being applied to a spectrogram of the millisecond pulsar B1937+21. 


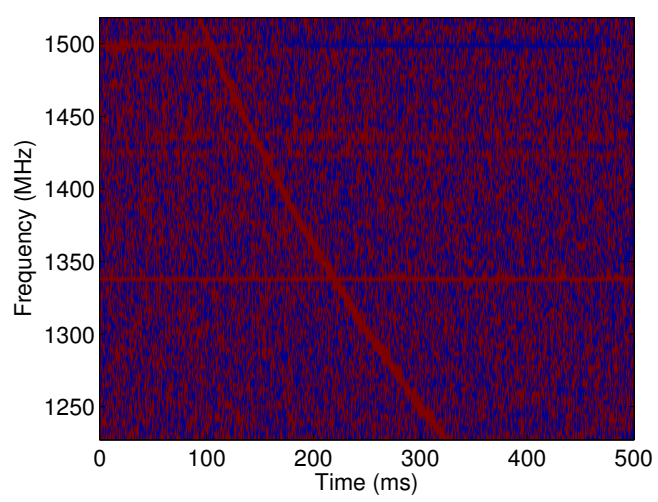

(a) Lorimer Burst

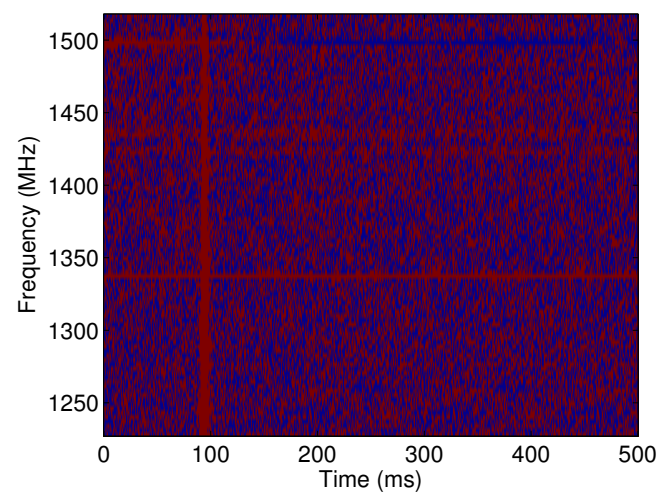

(c) De-dispersed of (a)

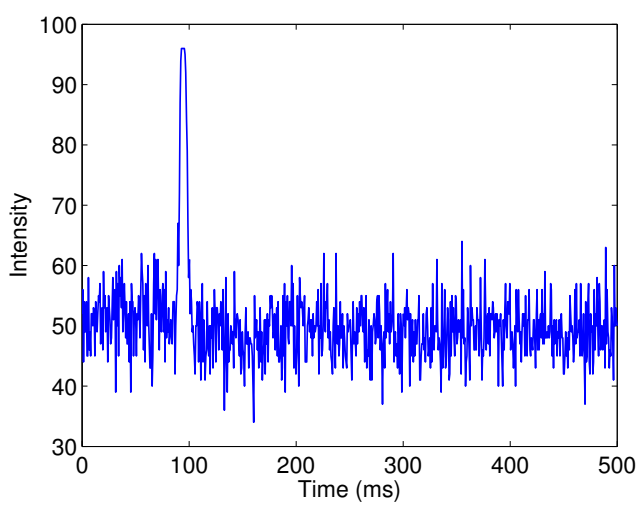

(e) Time Series of (c)

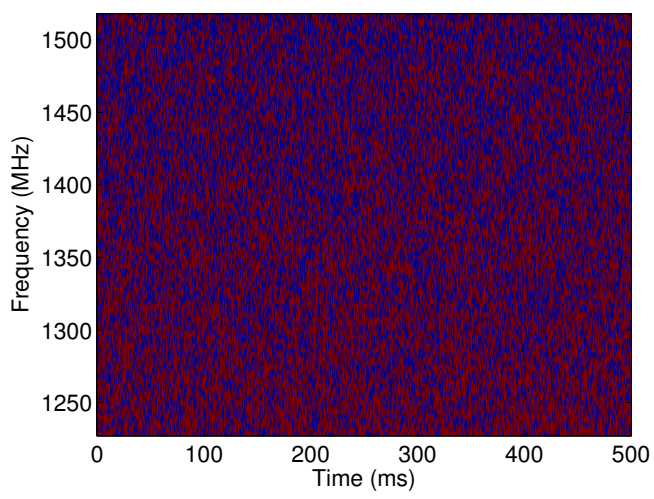

(b) Noise+RFI

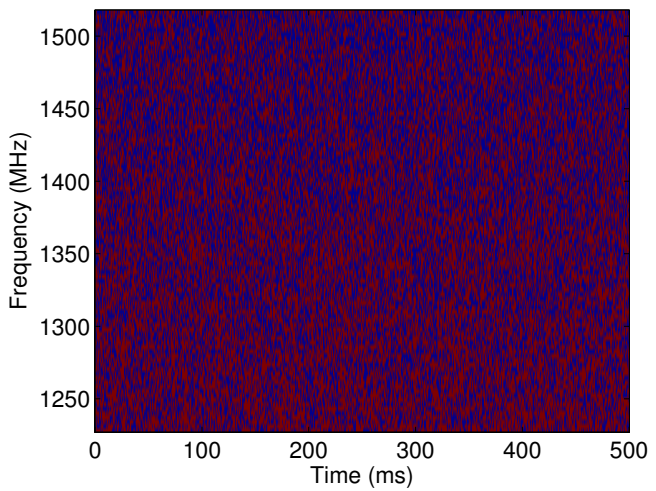

(d) De-dispersed of (b)

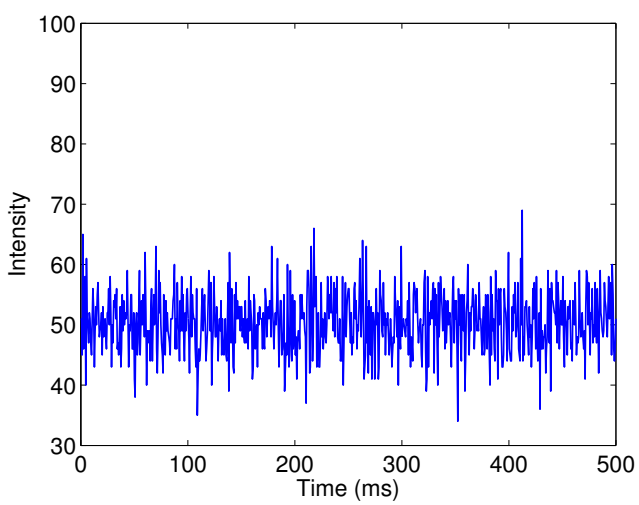

(f) Time Series of (d)

Figure 1.3: Main steps of the conventional detection approach applied to Lorimer Burst and Noise+RFI only filter bank data.

Recently, a number of fast transform-based detection algorithms capable of detecting isolated astronomical pulses of transients have been developed [27 30]. Their computational 


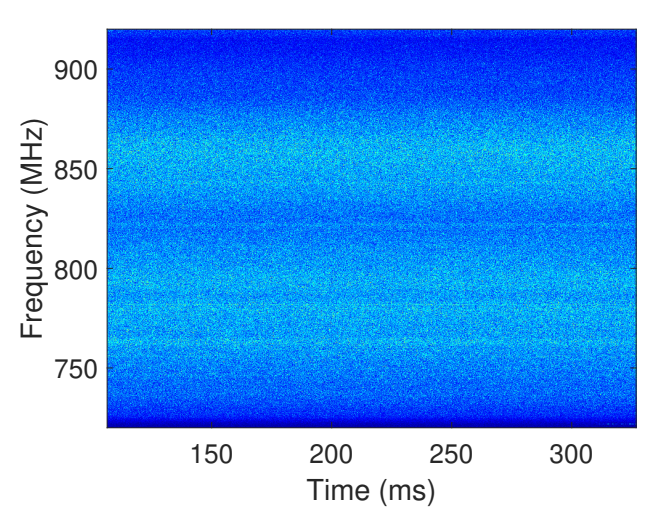

(a) B1937+21 Pulsar

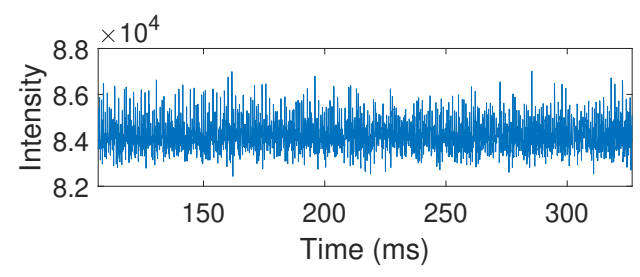

(c) Time Series of (b)

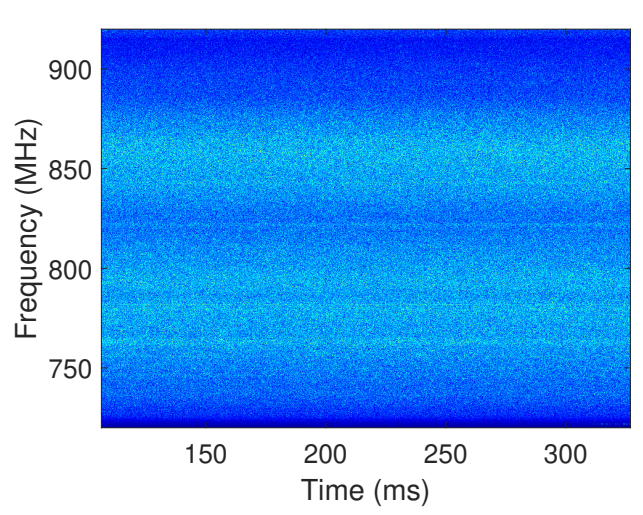

(b) De-dispersed of (a)

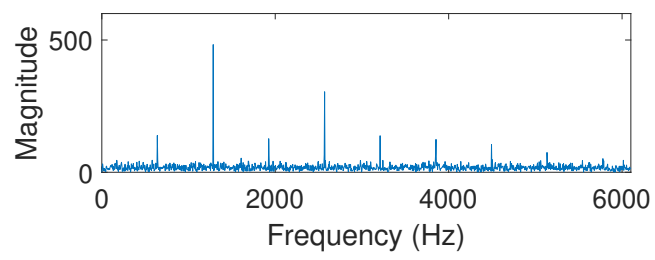

(d) FFT of (c)

Figure 1.4: Main steps of the conventional detection approach in application to a spectrogram of the millisecond pulsar B1937+21.

complexity is about $M \times N \log (N)$, where $M$ is the number of time samples and $N$ is the number of frequency channels in an analyzed spectrogram. Weber et al [27] suggested the application of Spatial Fourier transform to a spectrogram followed by a Hough transform as a means of detecting giant pulses emitted by neutron stars. The algorithm by Zackay et al. [28] is based on the application of the Hough transform [31] to a finite window extracted from a spectrogram. The energy of data is integrated along a bank of dispersion lines. The detection algorithm by Schmid et al. [30 is also applied to finite windows extracted from spectrograms. Spatial Fourier transform (SFT) is applied to each window and then is multiplied one by one with a bank of two-dimensional filters. The cumulative energy in the product is calculated and compared to a threshold.

Together with transform-based pulse detection algorithms, several algorithms utilizing deep learning techniques and deep convolutional neural networks have been developed (see for example Connor et al. [32], Zhang et al. [33], Agarwal et al. [34], and Zhang et al. [35]). The algorithms are fast, but require a large amount of training data as well as a carefully 
tuned convolutional neural network. In addition, the algorithms have to be retrained every time new set of data is involved.

While the outlined conventional approach to pulsar detection is well established, there is always an opportunity for the development of efficient alternative approaches.

\subsection{Dissertation Outline}

This dissertation consists of seven chapters. Chapter 2 presents a new algorithm based on the Radon transform applied to filter bank data followed by the Fourier transform. Spatial Fourier Transform (SFT) based detection method is presented in Chapter 3. The algorithm in Chapter 2 is updated in Chapter 4, where a linearizion step is applied to filter bank data prior to applying the two transformations. Similarly, the algorithm in Chapter 3 is further

extended in Chapter 5 by including a linearizion step prior to the SFT step. Chapter 6 considers a different set up. It assumes that in place of a single pixel telescope, data are collected by a Dense Aperture Array. The chapter presents two pulse detection algorithms under the assumed set up. Finally, in Chapter 7 several future directions are outlined. 


\section{Chapter 2}

\section{Detection of FRBs based on SFT and Radon Transform}

\section{$2.1 \quad$ Introduction}

This work is inspired by an approach described by Weber et al 27] which suggested the application of a two-dimensional Fourier transform followed by a Hough transform as a means of detecting giant pulses emitted by neutron stars. We propose a new alternative data-driven approach for the detection of isolated astrophysical pulses. The approach exploits the efficiency of two transform methods, the Radon transform and the Fourier transform. A Radon transform is applied to a broad band spectrogram - the astrophysical signal stored as a two-dimensional function of time and frequency (also known in radio astronomy as filter bank data). Hough transform can also be implemented instead of Radon transform. The output of the Radon transform is presented as a set of projections parameterized by an angle parameter. The Fourier transform is applied to each projection, and the cumulative magnitude Fourier transform in each projection is evaluated. The detection algorithm compares the total power in all projections to the left and right of $90^{\circ}$. Once an astrophysical pulse is detected, its DM value is estimated by means of a least square approach involving an analytical signature. Together with the least-square estimate of DM we develop two bounds on the DM value due to a linearization of the dispersion equation. The performance of the developed detection algorithm, the performance of the DM estimation approach and the bounds are demonstrated 


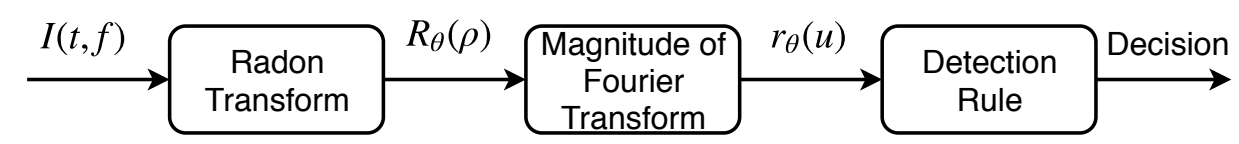

Figure 2.1: A block diagram of the proposed detection approach.

on simulated and practical data. The results demonstrate a great promise.

Part of the detection and estimation methodology outlined in this work was previously presented at the 2017 International Conference on Acoustics, Speech and Signal Processing [36]. Here we will emphasize the differences with the previous work while briefly summarizing the processing steps of the detection and estimation algorithms and allocate more space to the description of bounds and the results of performance analysis.

The remainder of the chapter is organized as follows. Section 2.2 provides details of the proposed detection algorithm. Section 2.3 describes a least square approach for estimation of the DM value. Section 2.4 introduces a lower and upper bounds on the DM value due to a linearization of the dispersion equation. Section 2.5 presents the results of performance analysis. A short summary is provided in Section 2.6 .

\subsection{Detection Algorithm}

The detection algorithm is composed of several consecutive signal processing steps. A block-diagram illustrating them is shown in Fig. 2.1. A brief description of each block in the block-diagram is provided below.

\subsubsection{Radon transform}

The first block in Fig. 2.1 applies the Radon transform to filter bank data. Denote by $I(t, f)$ the filter bank data (treated here as an image), then the Radon transform is mathematically defined [37] as

$$
R_{\theta}(\rho)=\int_{t_{i}}^{t_{f}} \int_{f_{l}}^{f_{h}} I(t, f) \delta(\rho-t \cos \theta-f \sin \theta) d t d f,
$$

where $\theta$ is the angle of a projection, $\rho$ is the offset of a projection line from the origin of the $(\mathrm{t}, \mathrm{f})$ plane, $t_{i}, t_{f}, f_{l}$ and $f_{h}$ define four time-frequency boundary points of the $(\mathrm{t}, \mathrm{f})$ plane and 


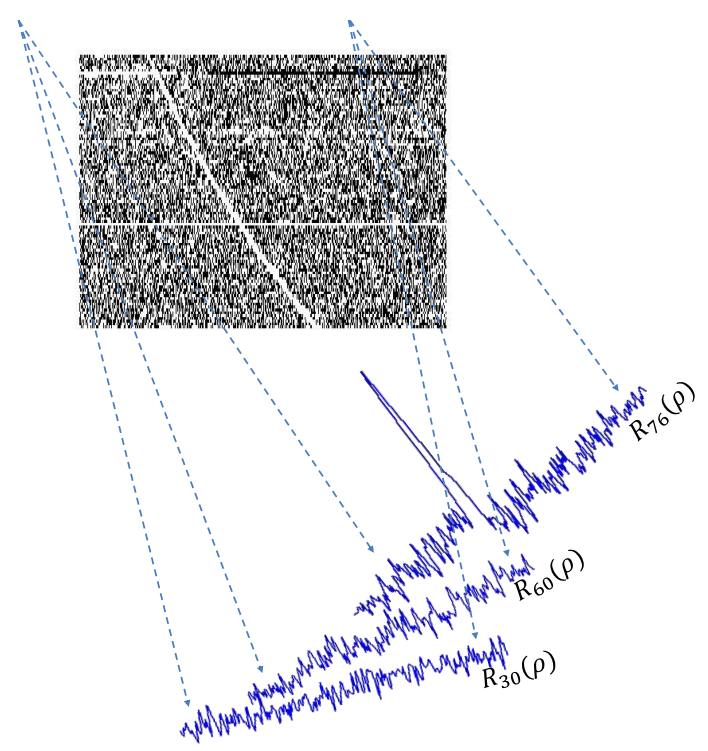

Figure 2.2: The result of applying the Radon transform to the Lorimer burst at three different orientations $\theta=30^{\circ}, \theta=60^{\circ}$, and $\theta=76^{\circ}$.

$\delta(\cdot)$ is the Dirac delta function. Assuming that an astrophysical pulse has a Gaussian profile in both directions, $I(t, f)$ can be expressed as

$$
I(t, f)=h \exp \left(-\frac{t^{2}}{2 \alpha^{2}}-\frac{f^{2}}{2 \beta^{2}}\right) * * \delta\left(t_{r e f}-t-4.15 \times 10^{3} \times \mathrm{DM} \times\left(f_{r e f}^{-2}-f^{-2}\right)\right),
$$

where $h$ is the peak of the pulse profile in a frequency channel, the parameters $\alpha$ is a spread of the Gaussian bell function in $t$ direction; $\alpha$ is related to the full width at half maximum (FWHM) according to FWHM $=2 \sqrt{2 \ln 2} \alpha$, the parameters $\beta$ is the spread of the Gaussian bell function in $f$ direction, $t_{r e f}$ is the reference time, $f$ is the radio frequency of a channel, measured in $\mathrm{MHz}, f_{r e f}$ is a reference frequency in $\mathrm{MHz}$ (related to $t_{r e f}$ ), DM stands for the dispersion measure in the units of $\mathrm{pc} / \mathrm{cm}^{3}$, and "**" stands for a two-dimensional convolution. In radio astronomy $f_{r e f}$ is often selected as the central frequency of the receiver bandwidth.

Figure 2.2 provides an example of applying the Radon transform to the "Lorimer burst" [3] at three different angles $\theta=30^{\circ}, \theta=60^{\circ}$, and $\theta=76^{\circ}$. The value of the projection at the origin $(\rho=0)$ corresponds to the integral of the pixel values along the line that passes through the middle of the image $I(t, f)$ in Fig. 2.3(a). Figure 2.3(c) shows the Radon transform of the Lorimer burst. Figure 2.3(d) displays the Radon transform of the filter bank 


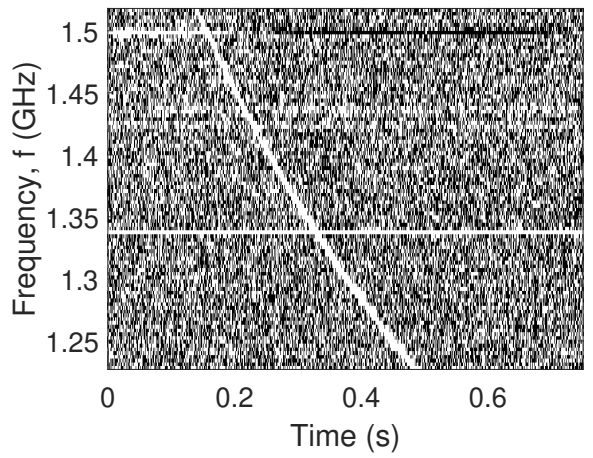

(a) Lorimer burst

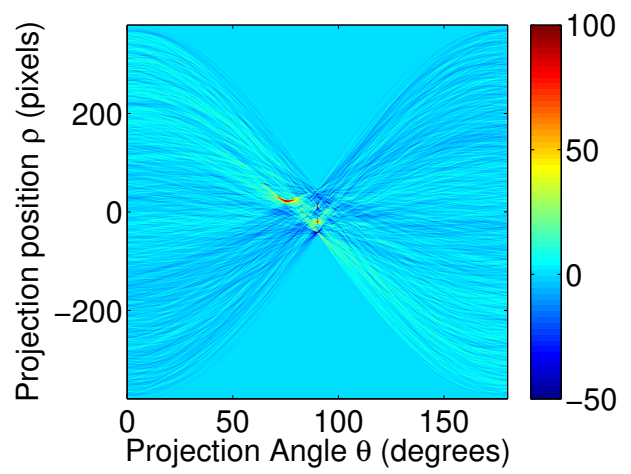

(c) Radon transform of (a)

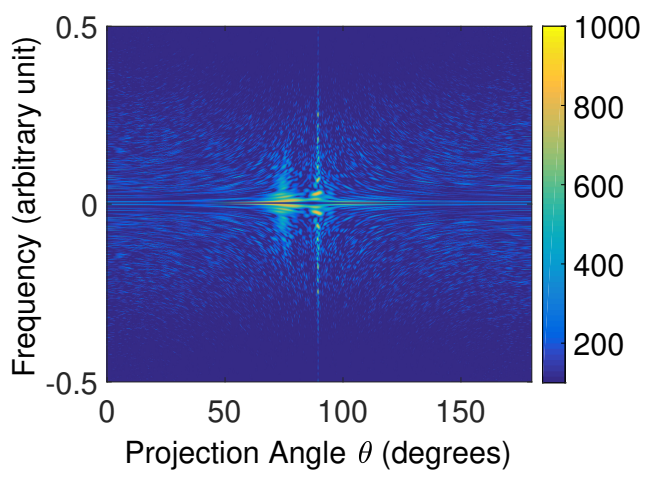

(e) magnitude FT for (c)

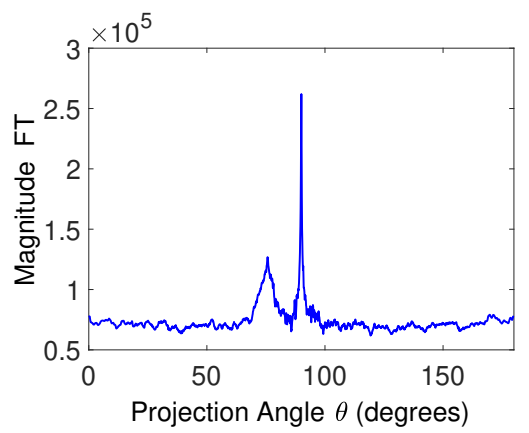

(g) integrated magnitude FT in (e)

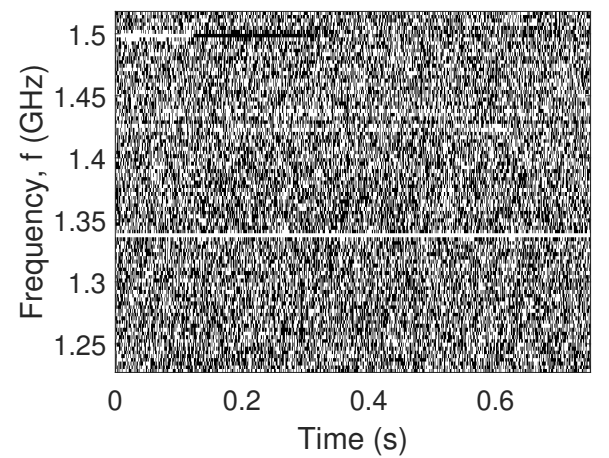

(b) noise + RFI

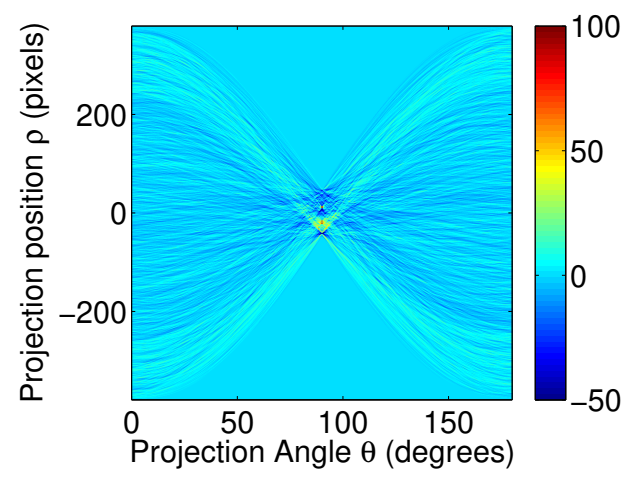

(d) Radon transform of (b)

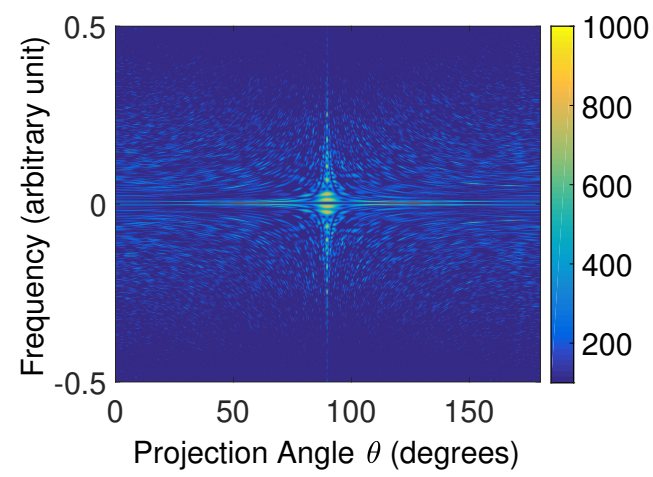

(f) magnitude FT for (d)

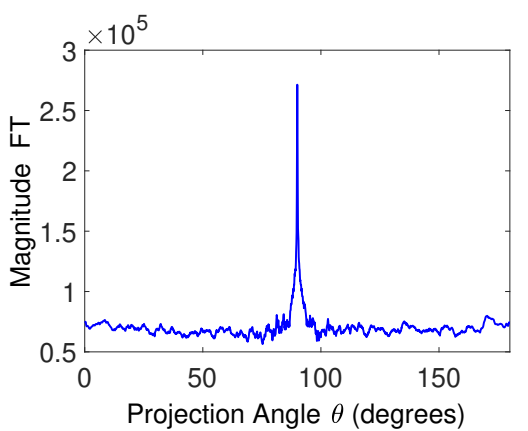

(h) integrated magnitude FT in (f)

Figure 2.3: Filter bank data in (a) and (b) and their step-wise transformations. 
data shown in panel (b), which presents noise plus radio frequency interference (RFI) - unwanted terrestrial signals - but no astrophysical pulse. The origin of the radon transform is at $\left(\rho=0, \theta=90^{\circ}\right)$.

\subsubsection{One dimensional Fourier transform}

As a second step, the algorithm applies the one dimensional Fourier transform along the columns of Radon transformed filter bank data. The Fourier transform for a projection of an image at angle $\theta$ can be found as

$$
r_{\theta}(u)=\int_{-\infty}^{\infty} R_{\theta}(\rho) \exp (-j 2 \pi u \rho) d \rho,
$$

where $u$ represents a frequency. Panels (e) and (f) in Fig. 2.3 show the magnitude Fourier transform of the images in panels (c) and (d), respectively.

\subsubsection{Cumulative magnitude Fourier transform}

Next, the algorithm integrates along the frequency dimension. By applying the FT, the signature of the pulse will be concentrated at low frequencies. This reduces the reach area for the signature of the pulse. The sum of magnitudes of the data over the columns of the images in panels (e) and (f) is shown in panels (g) and (h).

Figure 2.3(g) displays the cumulative magnitude Fourier transform as a function of the projection angle $\theta$. Note that the Lorimer burst is observed as a relatively strong and approximately Gaussian shaped pulse in the range of projection angles $\theta \in\left[71^{\circ}, 79^{\circ}\right]$. The range of angles is found at the Full Width Half Maximum (FWHM) of the Gaussian shaped pulse. A large spike at $\theta=90^{\circ}$ corresponds to RFI in the data. RFI is characterized by a zero DM value and may be broadband at a single time (bursty) or persistent at a single frequency. Such interference shows up at $\theta=0^{\circ}$ and $\theta=90^{\circ}$ in a plot of the magnitude Fourier transform. RFI has intentionally been left in the figures presented here to illustrate its effect. This is normally removed by our algorithm by simply suppressing the power in the projections around $0^{\circ}$ and $90^{\circ}$. The conventional pulse detection approach also removes RFI prior to the pulse detection process. 


\subsubsection{Decision rule}

The pulse can be detected by analyzing the cumulative magnitude Fourier transform in the projections. As observed from the panel (h) in Fig. 2.3, the signature of noise is nearly symmetric around $90^{\circ}$. From the panel $(\mathrm{g})$, the case when the data contain a pulse, the distribution of cumulative magnitude Fourier transform is skewed due to the dispersion relationship described by Eq. 2.2 .

Denote by $r_{\theta}$ the cumulative magnitude Fourier transform in the projection at an angle $\theta$. Then comparing the total $r_{\theta}$ in the projections below and above $90^{\circ}$ leads to the following intuitive decision rule. Decide a candidate pulse is present if

$$
\sum_{\theta=\epsilon}^{90^{\circ}-\epsilon}\left|r_{\theta}\right|^{2}>\gamma \sum_{\theta=90^{\circ}+\epsilon}^{180^{\circ}-\epsilon}\left|r_{\theta}\right|^{2},
$$

where $\epsilon$ is a small number introduced to avoid inclusion of RFI and $\gamma$ is a decision threshold $(\gamma>1)$. The candidate pulse is not detected, otherwise.

We can also take an alternative approach to the detection of pulses. Similar to the conventional algorithm [20], a matched filter (a rectangular pulse or a Gaussian shaped function) can be applied to data shown in the panels (g) and (h). Then the standard deviation of the filtered noise is estimated using the data to the right of the $90^{\circ}$ point. Pulse detection is performed by comparing the value of the peak of the filtered data to the left of the $90^{\circ}$ point against the value of the estimated standard deviation of the noise times a threshold $\gamma$. In the conventional detection algorithm the threshold is typically set to 10 .

\subsection{Estimation of DM value}

After a candidate pulse is detected, we have enough information to estimate its DM value. One of possible estimation approaches is to fit an analytically derived signature of an astronomical pulse into the Fourier transform of projection lines by means of least squares. An analytical signature is obtained by combining Equations 2.2 and 2.3

$$
r_{\theta}(u)=\int_{t_{i}}^{t_{f}} \int_{f_{l}}^{f_{h}} I(t, f) \int_{-\infty}^{\infty} \delta(\rho-t \cos \theta-f \sin \theta) e^{-j 2 \pi \rho u} d \rho d f d t .
$$


Integrating out $\rho$ results in

$$
r_{\theta}(u)=\int_{t_{i}}^{t_{f}} \int_{f_{l}}^{f_{h}} I(t, f) e^{-j 2 \pi(t \cos \theta+f \sin \theta) u} d t d f
$$

which is a two-dimensional Fourier transform with spatial frequencies replaced by $u \cos \theta$ and $u \sin \theta$. From Eq. 2.2 and the convolution property of the Fourier transform, Eq. 2.6 can be rewritten as

$$
\begin{array}{r}
r_{\theta}(u)=2 \pi h \alpha \beta \exp \left(-j 2 \pi u\left(t_{r e f}-\frac{d}{f_{r e f}^{2}}\right)\right. \\
\times \int_{f_{l}}^{f_{h}} \exp \left(-j 2 \pi\left(u \cos \theta \frac{\alpha^{2}}{f^{2}}+u \sin \theta\right)\right) d f
\end{array}
$$

where $d=4.15 \times 10^{3} \times D M$. Then the least square solution to the estimation problem is given as

$$
\widehat{\mathrm{DM}}=\arg \min _{\mathrm{DM} \in\left[\mathrm{DM}_{\min }, \mathrm{DM}_{\max }\right]}\left\|x_{\theta}(u)-r_{\theta}(u)\right\|^{2},
$$

where the distance is evaluated over all values $\theta$ and $u$ such that the estimated signal power in the data $\left|x_{\theta}(u)\right|^{2}$ exceeds the estimated noise variance $\sigma^{2}$ in the data.

\subsection{Bounds on DM Value}

In addition to the least-squares estimate of DM, we can develop a set of basic bounds on the DM value. An upper and lower such bounds are obtained by analyzing the plot of the magnitude Fourier transform as a function of projection angle (see Fig. 2.3(e)). The signature of the pulse in Fig. 2.3(e) can be approximated by a Gaussian curve with $\theta_{\text {lower }}$ and $\theta_{\text {upper }}$ as the locations of the half maximum of the function. These two angles are used to calculate the bounds on DM. Note that in the spectrogram plot of a pulse, tangents of $\theta_{\text {upper }}$ and $\theta_{\text {lower }}$ can be obtained by drawing two tangent lines, one touching the dispersed pulse at the point $\left(t_{h}, f_{h}\right)$ and the other one touching it at $\left(t_{l}, f_{l}\right)$, where $t_{l}$ and $t_{h}$ are the time instances of the pulse arrival to the lowest $f_{l}$ and the highest $f_{h}$ frequencies of the receiver band. The equations of the two lines are given as:

$$
\left(f_{h}-f\right) \tan \left(\theta_{\text {lower }}\right)=t-t_{h}
$$


and

$$
\left(f-f_{l}\right) \tan \left(\theta_{\text {upper }}\right)=t_{l}-t,
$$

where $\tan \left(\theta_{\text {lower }}\right)$ and $\tan \left(\theta_{\text {upper }}\right)$ are tangents to the dispersion line at the highest and lowest frequencies of the receiver band. Note that to bring angles $\theta_{\text {lower }}$ and $\theta_{\text {upper }}$ in correspondence with those identified in Fig. 2.3(e), we have to express time and frequency in discrete (or sampled) units. Denote by $T_{s}$ the sampling interval and by $K$ the number of discrete frequency channels in a spectrogram.

Setting $f$ in Eq. 2.9 to $f_{l}$ and $t$ to $t\left(f_{l}\right)$, the time instant when the pulse arrives to $f_{l}$ due to the linear approximation, the approximation delay is

$$
t\left(f_{l}\right)-t_{h}=\widehat{\mathrm{DM}}_{\text {lower }} \times 4150 \times\left(f_{l}^{-2}-f_{h}^{-2}\right) .
$$

From Equations 2.9 and 2.11, the lower bound on the DM value is easily obtained as

$$
\widehat{\mathrm{DM}}_{\text {lower }}=\frac{K T_{s} \tan \left(\theta_{\text {lower }}\right)}{4150\left(f_{l}^{-2}-f_{h}^{-2}\right)}
$$

Similarly, from Eq. 2.10 and the dispersion equation for the interval $t_{l}-t\left(f_{h}\right)$ given as

$$
t_{l}-t\left(f_{h}\right)=\widehat{\mathrm{DM}}_{\text {upper }} \times 4150 \times\left(f_{l}^{-2}-f_{h}^{-2}\right) .
$$

we arrive to the following upper bound on the DM value:

$$
\widehat{\mathrm{DM}}_{\text {upper }}=\frac{K T_{s} \tan \left(\theta_{\text {upper }}\right)}{4150\left(f_{l}^{-2}-f_{h}^{-2}\right)}
$$

\subsection{Performance Analysis}

In this section the performance of the detection and DM estimation algorithms is evaluated. The performance of the detection algorithm is demonstrated through Detection Rate at zero empirical False Alarm Rate [38. Due to a very limited amount of practical data containing real FRB pulses, our numerical evaluation involving FRBs mostly relies on simulated data.

RRAT series used in this evaluation were provided by the Department of Physics and Astronomy at West Virginia University [12]. 
Table 2.1: A list of fast radio bursts used in our numerical analysis.

\begin{tabular}{|c|c|c|c|c|c|}
\hline Name & Telescope & $\begin{array}{c}\text { DM } \\
\left(\mathrm{pc} / \mathrm{cm}^{3}\right)\end{array}$ & $\begin{array}{c}\text { Width } \\
(\mathrm{ms})\end{array}$ & SNR & $\begin{array}{c}\text { Detection } \\
\text { Rate }\end{array}$ \\
\hline \hline FRB121102 & Arecibo & 557 & 3 & 14 & $100 \%$ \\
\hline FRB110220 & Parkes & 944.38 & 5.6 & 49 & $100 \%$ \\
\hline FRB160608 & UTMOST & 682 & 9 & 12 & $100 \%$ \\
\hline FRB170107 & ASKAP & 609 & 2.6 & 16 & $100 \%$ \\
\hline FRB180301 & Parkes & 520 & 3 & 16 & $100 \%$ \\
\hline FRB180311 & Parkes & 1575.6 & 12 & 11.5 & $100 \%$ \\
\hline
\end{tabular}

\subsubsection{Numerical Evaluation based on Simulated Data}

The initial data are displayed in filter bank format, where astrophysical pulses are dispersed according to Eq. 2.2. We assume that the pulse profile of each astrophysical pulse is Gaussian with the FWHM set to correspond to the width of pulse of interest. The bandwidth of the simulated filter bank data, sampling interval, the number of discrete frequency channels, the pulse SNR, and the DM value of a simulated pulse are set to the values provided in [5]. To add noise to a simulated astrophysical pulse, we apply the conventional definition of the SNR, where it is defined as a ratio of the peak value of a de-dispersed and matched-filtered observed signal, to the root mean square (RMS) of the simulated noise. A detailed description of our simulation approach is provided in [30].

\section{Pulse Detection}

To analyze the detection performance of the developed algorithm we simulate the FRBs listed in Table 2.1, we generate 100 realizations per FRB. For each FRB we also generate 100 spectrograms of pure noise. The decision rule (see Eq. 2.4) is applied to both noisy data containing a pulse and to the data containing noise only. The value of parameter $\gamma$ is selected such that the estimated False Alarm Rate (based on 100 realizations of pure noise) is zero. The last column in Table 2.1 presents the Detection Rate for each analyzed FRB. From this example, the developed algorithm shows promise in terms of detecting astrophysical pulses parameterized by a large DM value. Also note that the algorithm is able to detect both wide 
and narrow pulses as well as pulses down to SNR of 11.5.

\section{Effect of pulse width on the detection performance}

The results of previous subsection demonstrate that pulses parameterized by SNR above 11.5, width between $2.6 \mathrm{~ms}$ and $12 \mathrm{~ms}$ and DM value up to $1575.6 \mathrm{pc} / \mathrm{cm}^{3}$ are detectable by the developed algorithm. In this subsection we explore the effect of the pulse width on the detection performance. Our claim is that for a given DM and SNR values, our algorithm is much more sensitive to wide pulses than to narrow pulses.

To demonstrate this, we simulated astrophysical pulses for two values of DM, $300 \mathrm{pc} / \mathrm{cm}^{3}$ and $1500 \mathrm{pc} / \mathrm{cm}^{3}$, and several values of the pulse width. The input SNR in all simulations was set to 8 . Figures 2.4 and 2.5 show the dependence of the estimated SNR on the pulse width for the DM value of $300 \mathrm{pc} / \mathrm{cm}^{3}$ and $1500 \mathrm{pc} / \mathrm{cm}^{3}$, respectively. The pulses marked with a solid blue circle were detected by the proposed algorithm while those marked with a red diamond were not detected (missed). Since the input SNR for this experiment was set to 8 , it is reasonable to compare the detection performance of the proposed algorithm with that of the conventional algorithm with the threshold set to 8 . Note that most of pulses missed in both cases are of width below $5 \mathrm{~ms}$. Nearly all pulses of width greater than $5 \mathrm{~ms}$ were detected by the proposed algorithm. In general, the case of DM $=1500 \mathrm{pc} / \mathrm{cm}^{3}$ is a more challenging case for our algorithm compared to the case of DM $=300 \mathrm{pc} / \mathrm{cm}^{3}$. This was expected due to a much large number of noisy pixels in spectrograms at a larger DM value compared to the case of a small DM value.

The results shown in Figures 2.4 and 2.5 clearly indicate that wide pulses are easily detectable by the proposed algorithm, while the conventional algorithm seems to lose the ability to detect wide pulses. This conclusion is intuitive. Wide pulses are more noticeable in Fourier transformed data, since the energy of a wide pulse is concentrated in lower frequencies, while a narrow pulse requires a broader range of frequencies to describe it.

\section{DM Estimation}

The DM estimation approach outlined in Sec. 2.3 is applied to the simulated data described above. Figure 2.6 displays six plots of the estimated DM values versus realization 


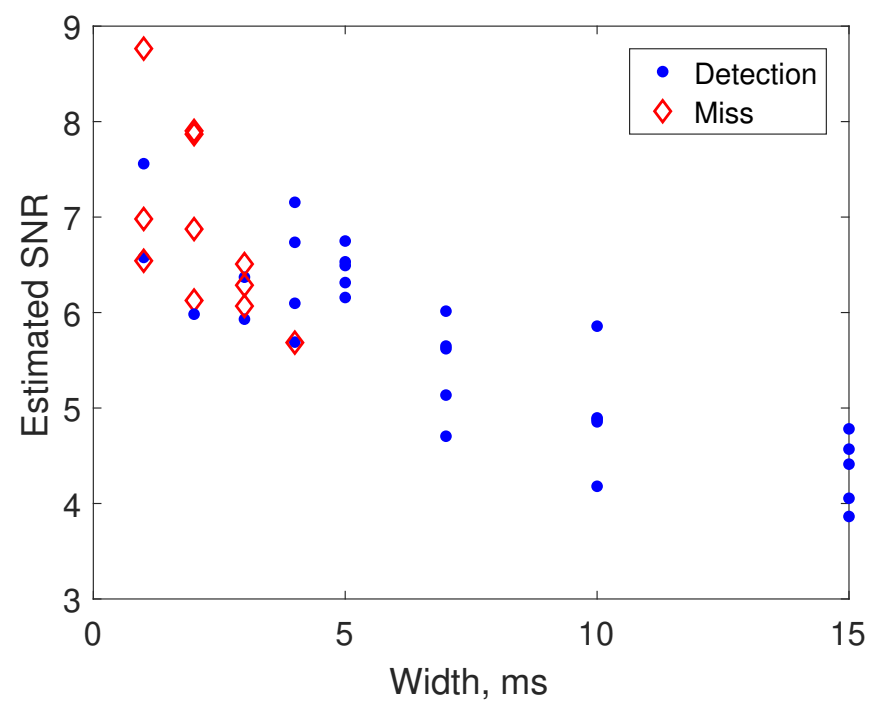

Figure 2.4: A sensitivity plot displaying the dependence of the estimated SNR values using the conventional algorithm versus the width of pulses. The input $\mathrm{SNR}=8$ and $\mathrm{DM}=300$ $\mathrm{pc} / \mathrm{cm}^{3}$.

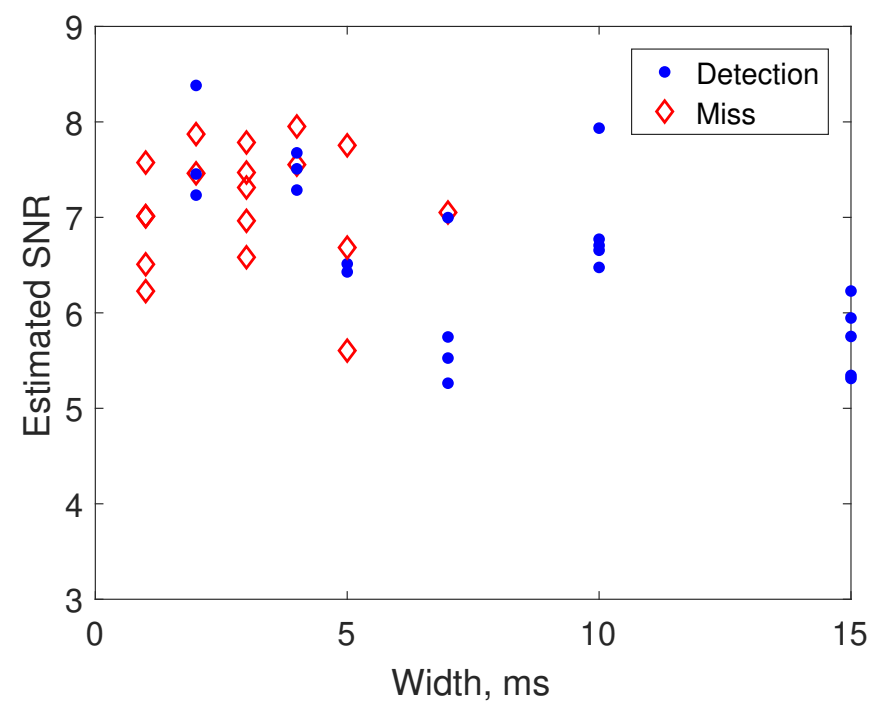

Figure 2.5: A sensitivity plot displaying the dependence of the estimated SNR values using the conventional algorithm versus the width of pulses. The input SNR $=8$ and $\mathrm{DM}=1500$ $\mathrm{pc} / \mathrm{cm}^{3}$. 
number. The estimated values are marked with red dots. Each plot also shows the bounds on the DM value due to Equations 2.11 and 2.13 marked with blue and green triangles. The horizontal line indicates the true DM value of each FRB as listed in [5].

A close inspection of each plot in Fig. 2.6 shows that the true DM value for each FRB lies between $\widehat{\mathrm{DM}}_{\text {lower }}$ and $\widehat{\mathrm{DM}}_{\text {upper }}$. Due to the fact that the estimate in Eq. 2.8 is a nonlinear function in $\theta$, the $\widehat{\mathrm{DM}}_{\text {upper }}$ values are more spread compared to $\widehat{\mathrm{DM}}_{\text {lower }}$. The estimates due to Eq. 2.8 are well clustered around the true DM value. These results indicate that the estimation approach in Sec. 2.3 and especially bounds in Equations 2.11 and 2.13 can be used as complimentary to the conventional detection algorithm. To reduce the time of blind de-dispersion used by the conventional algorithm, the proposed least square estimate of DM and the bounds on DM can provide an initial search point for the conventional algorithm.

\subsubsection{Numerical Evaluation based on Practical Data}

In this section we intend to apply the detection and DM estimation approaches described in Sections 2.2 and 2.3 to practical data. We first analyze the performance of the proposed algorithms in application to Masui pulse followed by performance analysis of the algorithms applied to series of seven RRATs.

\section{FRB 110523}

FRB 110523 was detected by Masui et al. [26] during their analysis of a data archive collected for the GBT neutral hydrogen 21-cm intensity mapping survey [39]. Prior to applying the proposed algorithm to the data, we isolated the pulse from the rest of the provided data then cleaned of RFI, and removed high frequency components; the result of this preprocessing is shown in Fig. 2.7(a).

The data are further transformed by applying the Radon transform as shown on panel (b) in Fig. 2.7 and then Fourier transformed along the columns, shown on panel (c) in Fig. 2.7. After the integration along the frequency channels, the magnitude Fourier transform profile as a function of the projection angle $\theta$ is obtained, shown in Fig. 2.7(d). Note that the signature of the Masui pulse appears as a relatively strong and approximately Gaussian 


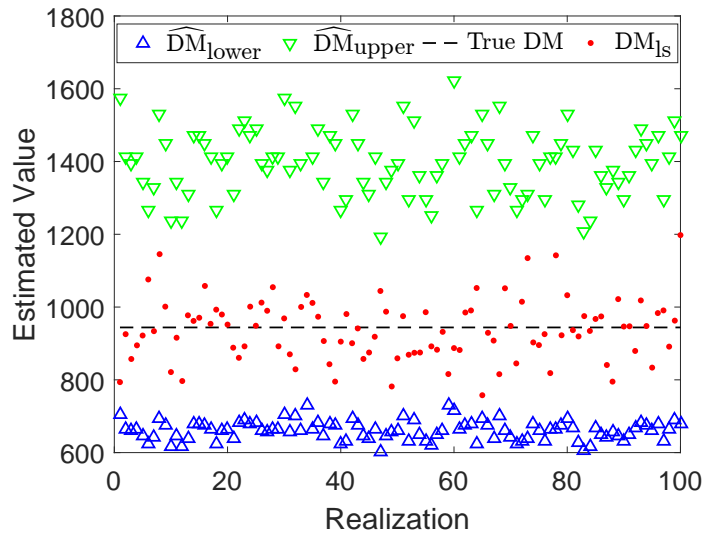

(a) FRB 110220

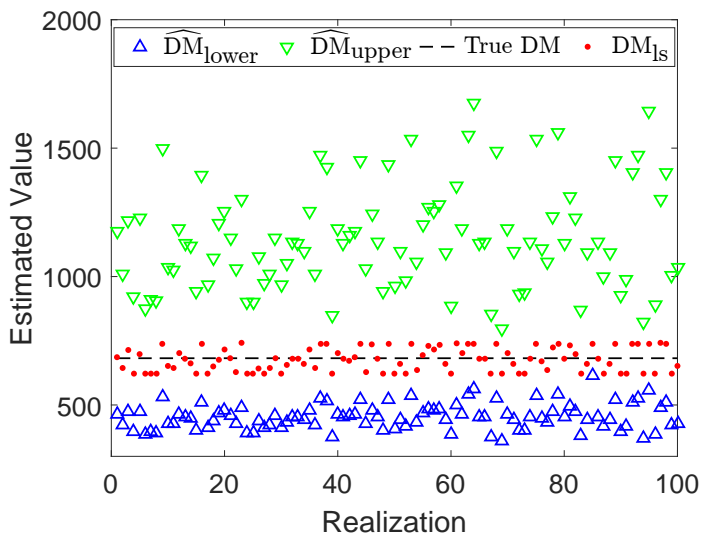

(c) FRB 160608

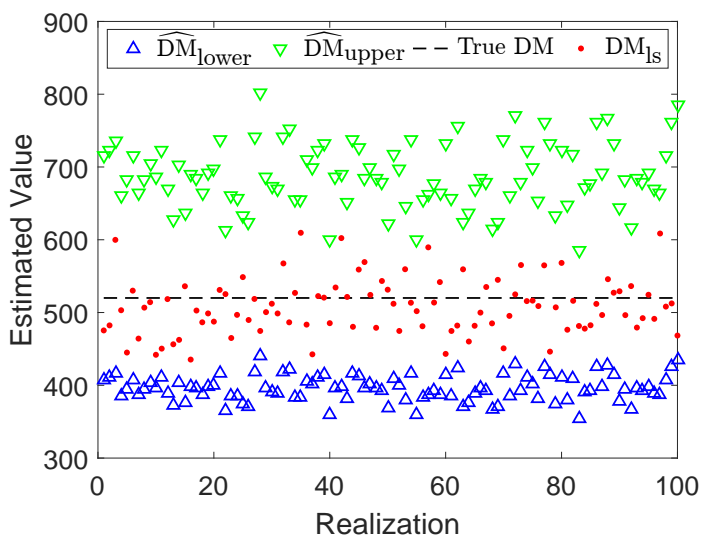

(e) FRB 180301

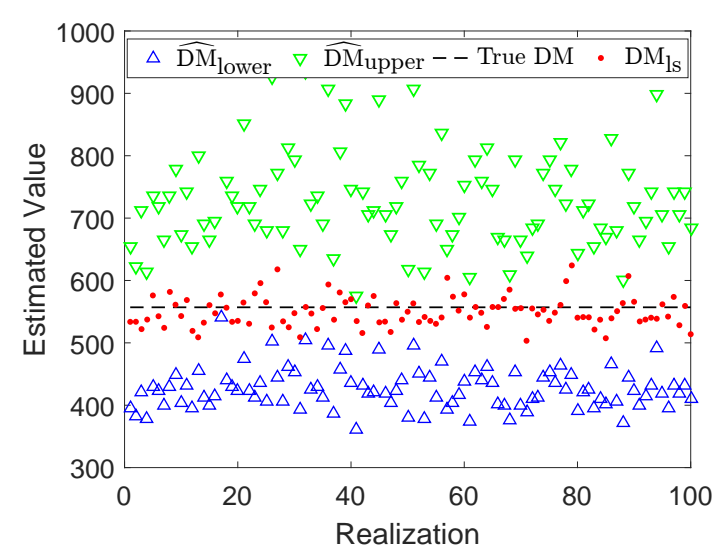

(b) FRB 121102

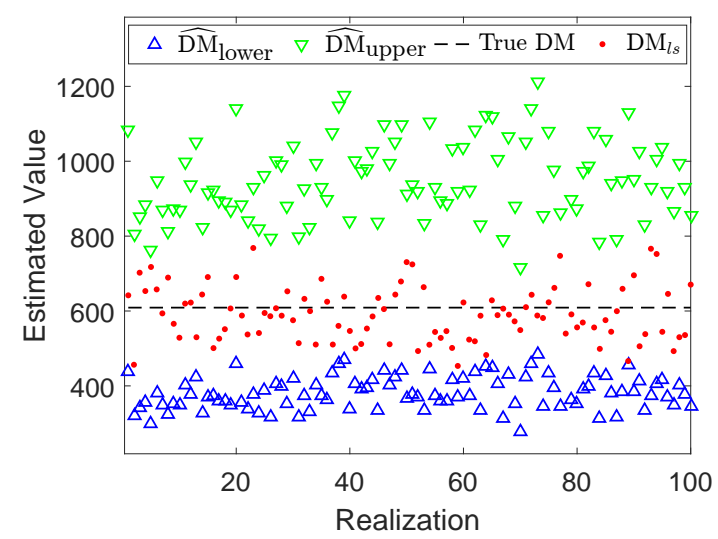

(d) FRB 170107

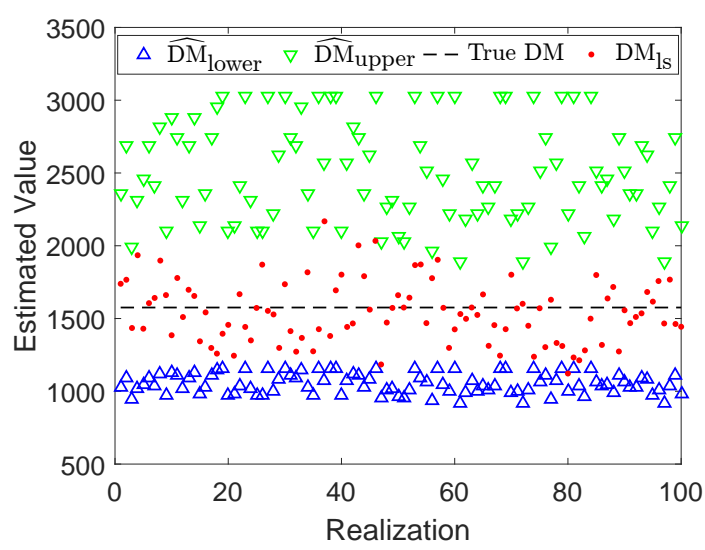

(f) FRB 180311

Figure 2.6: Shown are interval estimates of the DM value of the FRBs listed in Table 2.1. Each panel presents a plot of the estimated DM value versus the realization number, the true DM value and the DM values due to the bounds in Equations 2.12 and 2.14 
shaped pulse in the range of projection angles $\theta \in\left[57^{\circ}, 73^{\circ}\right]$.

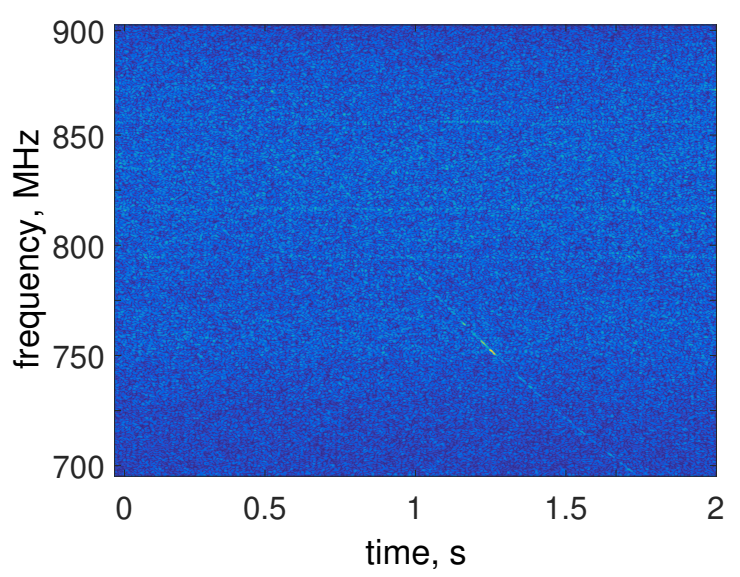

(a) Masui pulse

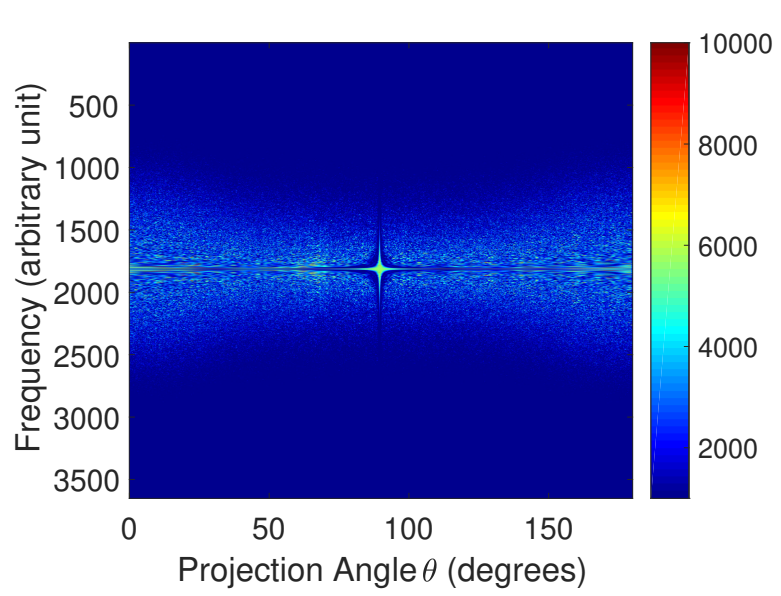

(c) magnitude FT for (b)

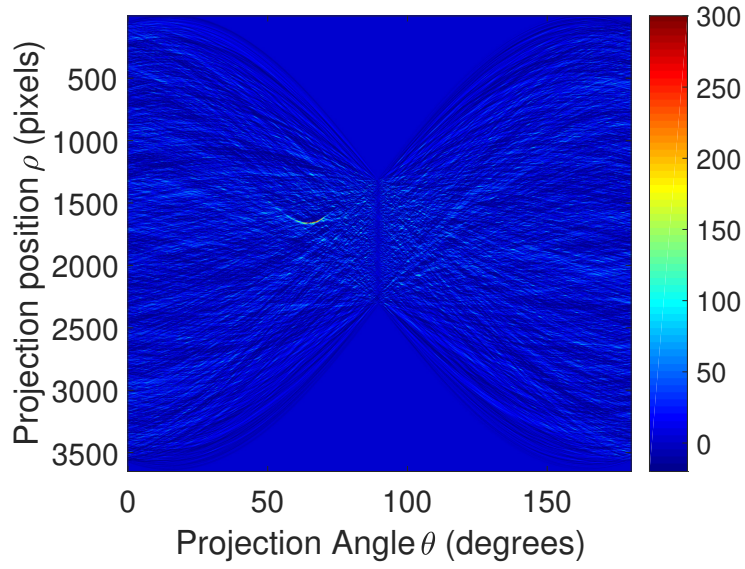

(b) Radon transform of (a)

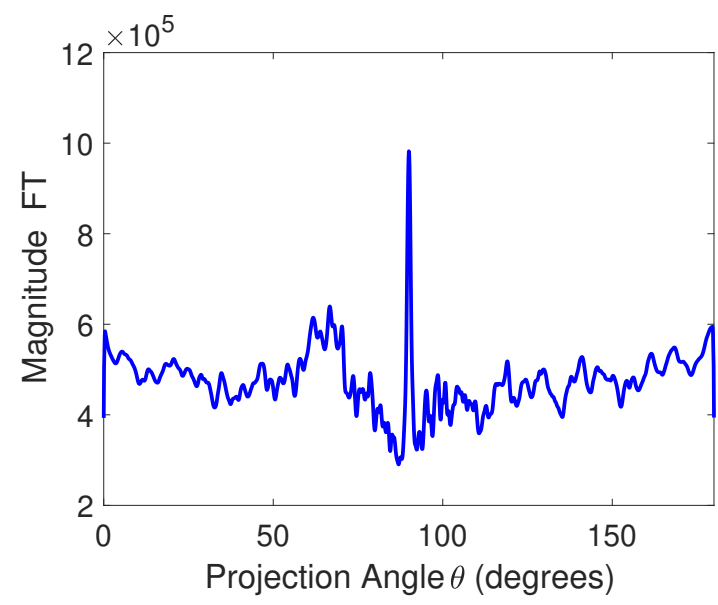

(d) integrated magnitude FT in (c)

Figure 2.7: Masui pulse data in (a) and its step-wise transformations in (b) through (d).

From Equations 2.11 and 2.13 we find the lower and upper bounds on the DM value of the Masui pulse. They are $\widehat{\mathrm{DM}}_{\text {upper }}=845.5 \mathrm{pc} / \mathrm{cm}^{3}$ and $\widehat{\mathrm{DM}}_{\text {lower }}=558.1 \mathrm{pc} / \mathrm{cm}^{3}$, respectively. The least square estimate and the true DM value for the Masui pulse are 682 and 623.36 $\mathrm{pc} / \mathrm{cm}^{3}$ [26], respectively. Note that both the estimate and bounds are reasonably close in their values to the true value of DM and thus either of them can be used as an initial value for a more refined DM estimation using the conventional algorithm. 


\section{RRAT series}

In addition to the analysis above, we tested the proposed detection and estimation algorithms on data of seven RRATs collected at the Parkes Telescope. The temporal length of raw time series varies from 20 minutes to 30 and 60 minutes. The data were converted into spectrograms each having 512 frequency channels and a temporal sampling interval $100 \mu s$. The Parkes receiver was tuned to acquire data in the range of frequencies between $1262 \mathrm{MHz}$ and $1518 \mathrm{MHz}$. The seven RRAT series used for performance analysis are listed in Table 2.2 together with a few parameters describing each individual RRAT. These series have been previously analyzed by applying the conventional algorithm. The count of pulses detected by the conventional algorithm (and confirmed through manual inspection and diagnostic plots) is presented in the fifth column of Table 2.2. Pulses were detected at SNR=5.

Before applying the proposed detection and estimation algorithms to the RRAT series, we applied some minor preprocessing to the data. Given a value of the period of a RRAT and the time of arrival of a strong pulse, as provided by the conventional algorithm, we used this information to partition each RRAT filter bank data into windows such that each window contains either noise or an entire noisy pulse. The proposed detection algorithm was applied to every window in the partitioned filter bank data.

The proposed algorithm confirmed every pulse detected by the conventional algorithm. The count of detected pulses is listed in the fifth column of Table 2.2. It also detected additional candidates, not previously detected by the conventional algorithm. The count of new candidates is listed in the sixth column of the table. Note that the table also reports the false alarm rate for every analyzed RRAT series. It is evaluated based on data after all windows with potential pulses were removed. Potential pulse locations were determined based on the time of arrival (TOA) of pulses reported by the conventional detection algorithm and also by taking into account the knowledge of the period of every RRAT.

Figure 2.8(a) displays the least square estimates of the DM value for the pulses of RRAT J1819-1458 detected by the conventional algorithm from file RR0037_0151. Together with the estimated values of the DM for each of 49 pulses we show the upper and lower bounds on the DM value. The true DM value for this RRAT is $196 \mathrm{pc} / \mathrm{cm}^{3}$. Panels (b)-(d) in Fig. 
Table 2.2: A list of RRATs used in our numerical analysis.

\begin{tabular}{|c|c|c|c|c|c|c|}
\hline $\begin{array}{c}\text { Name of } \\
\text { RRAT }\end{array}$ & Data File & $\begin{array}{c}\text { DM } \\
\left(\mathrm{pc} / \mathrm{cm}^{3}\right)\end{array}$ & $\begin{array}{c}\text { Width } \\
(\mathrm{ms})\end{array}$ & $\begin{array}{c}\text { \# Detected } \\
\text { pulses }\end{array}$ & $\begin{array}{c}\text { \# New } \\
\text { candidates }\end{array}$ & $\begin{array}{c}\text { FA } \\
\text { Rate }\end{array}$ \\
\hline \hline J1819-1458 & RR0037_0151 & 196 & 3 & 48 & 1 & $3.8 \%$ \\
\hline J1317-5759 & RR0037_0071 & 145.4 & 10 & 13 & 1 & $0.1 \%$ \\
\hline J1913+1330 & RR0086_0181 & 175.64 & 2 & 7 & 0 & $0 \%$ \\
\hline J1839-0136 & RR0037_0031 & 293.4 & 15 & 1 & 0 & $0 \%$ \\
\hline J1826-1419 & RR0090_0121 & 159 & 2 & 16 & 1 & $6 \%$ \\
\hline J1047-58 & RR0057_0251 & 69.3 & 4 & 27 & 17 & $1.06 \%$ \\
\hline J1423-5647 & RR0070_0131 & 32.9 & 26.5 & 1 & 4 & $0.127 \%$ \\
\hline
\end{tabular}

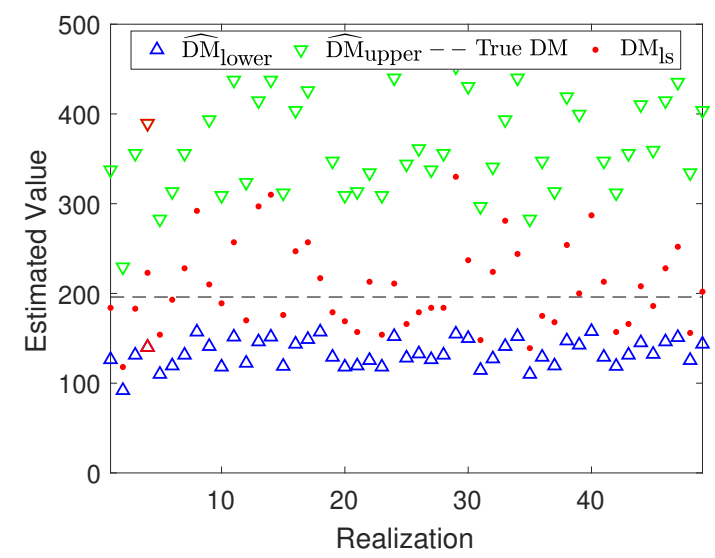

(a) File RR0037_0151 for RRAT J1819-1458

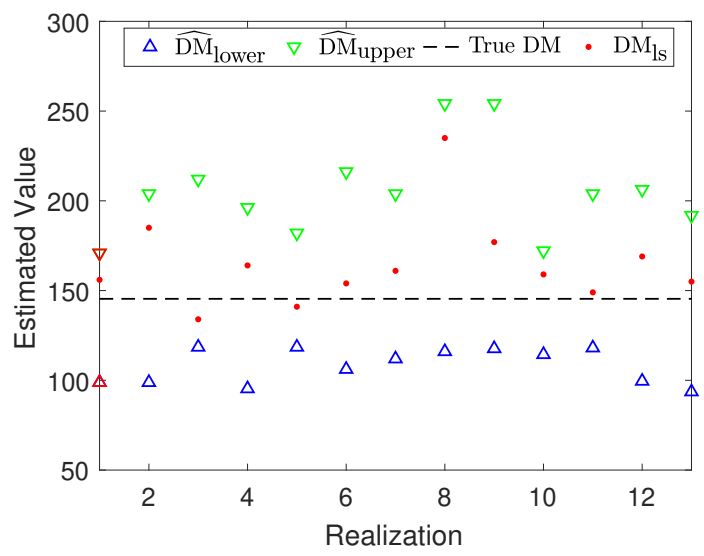

(a) File RR0037_0071 for RRAT J1317-5759

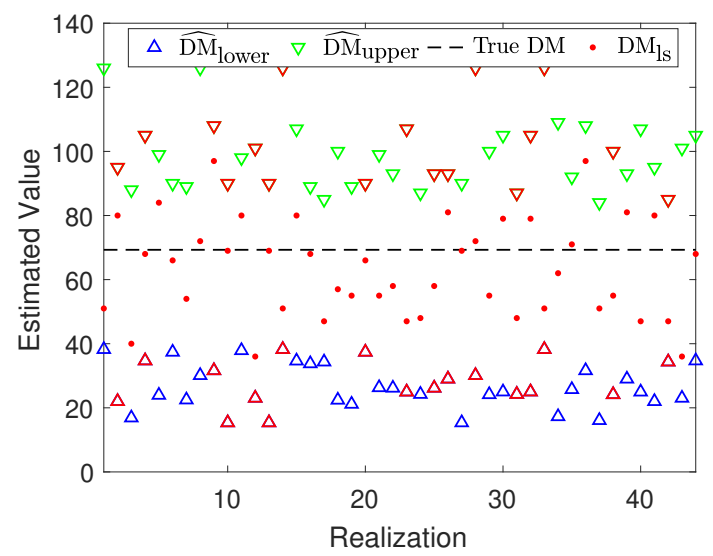

(b) File RR0057_0251 for RRAT J1047-58

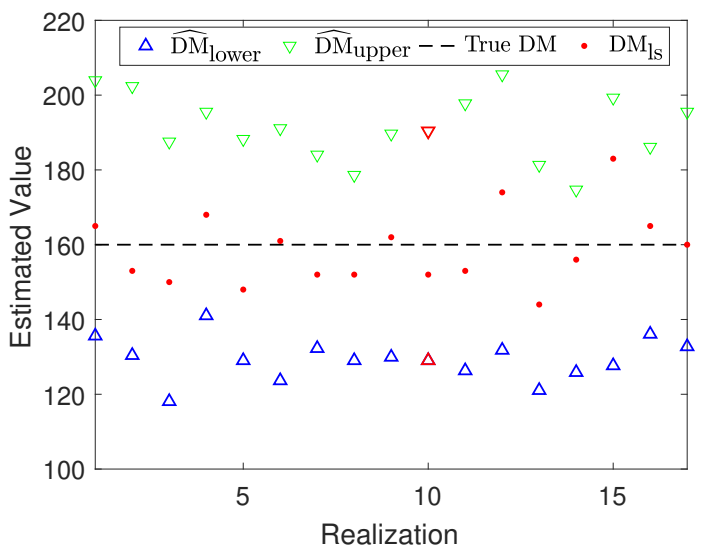

(b) File RR0090_0121 for J1826-1419

Figure 2.8: Estimated DM values for the detected pulses of four RRAT series. Bounds on the DM value of the new candidate pulses are marked with red triangles. 
2.8 demonstrate similar results for files RR0037_0071, RR0057_0121, and RR0057_0251, respectively. The pulses with bounds marked with red triangles are new candidates, which were not previously detected by the conventional algorithm. Note that the estimated DM of the new candidates as well as the bounds are within acceptable range of estimates and bounds for the pulses of the four RRATs.

\subsection{Summary}

This chapter introduced a data driven detection algorithm for the detection of isolated astrophysical pulses. The algorithms operates on spectrograms. It detects astronomical pulses using information contained in spectrogram only, without appealing to training or any additional data. The algorithm involves two transformations, the Radon transform followed by the Fourier transform, and comprises three main steps. In step one, we apply the Radon transform to the spectrogram. In step two, the Fourier transform is applied to columns of the data in part one and the total power in each projection (parameterized by an angle parameter) is evaluated. In step three we apply the mean-square error approach to compare the difference in power of the data to the left and right of $90^{\circ}$. Compared to the conventional detection algorithm used in radio astronomy for the detection of isolated pulses, the proposed detection algorithm does not require any searching for the value of the unknown DM, which is computationally expensive. In addition, no RFI removal is required prior to applying the proposed detection algorithm.

Both practical and simulated data were used to illustrate the performance of the detection algorithm. The results of our simulations demonstrated that many of the recently discovered FRBs would be readily detected by the developed approach. Our experiments involving simulated data also indicated that the proposed detection algorithm is more sensitive to wide pulses compared to the conventional pulse detection algorithm. Apart from the simulated data, we tested the proposed detection algorithm on practical data including Masui pulse and time series of seven RRATs. Masui pulse is clearly visible by eye in the transformed data and detectable by the proposed algorithm. Applied for the RRAT series, the proposed detection algorithm was able to identify all pulses previously reported in the literature and 
it was also able to find a number of new candidate pulses.

Once an astrophysical pulse is detected, we applied a least squares approach to estimate the DM value of the pulse. The results of performance analysis demonstrated that the estimated DM value is in the average $50 \mathrm{pc} / \mathrm{cm}^{3}$ apart from the true DM value (with 150 $\mathrm{pc} / \mathrm{cm}^{3}$ being the maximal deviation). In addition to the least-square estimation of the DM we developed the upper and lower bounds on the DM value. The bounds are not tight, but useful. Either of the two bounds as well as the least square estimate can be used as an initial $\mathrm{DM}$ value for a more refined estimation of the DM value due to conventional algorithm. 


\section{Chapter 3}

\section{Detection of Periodic Astrophysical}

\section{Pulses}

\subsection{Introduction}

This chapter presents a Fourier transform based approach for the detection and analysis of periodic astrophysical signals. In place of de-dispersing (which is a time consuming operation) and integrating data over frequency channels to yield a one-dimensional signal, we apply SFT to filter bank data, then detect periodic astrophysical signals and analyze their parameters such as DM and rotational period. This approach allows searching for periodic astrophysical signals in real time. Its complexity is dominated by the complexity of the SFT, that is, $N K \log (N K)$ with $N$ being the number of temporal samples in an observation window and $K$ being the number of frequency channels.

\subsection{SFT Signature of a Periodic Signal}

As recently demonstrated SFT can be used as an alternative and also complimentary (to the conventional) approach to detect isolated astrophysical pulses [40]. An example of a single simulated pulse in filter-bank format is shown in Fig. 3.1(a). Its SFT signature is shown in Fig. 3.1(c). By analogy, the application of SFT to a periodic astrophysical signal shown in Fig. 3.1(b) yields the signature in Fig. 3.1(d). 


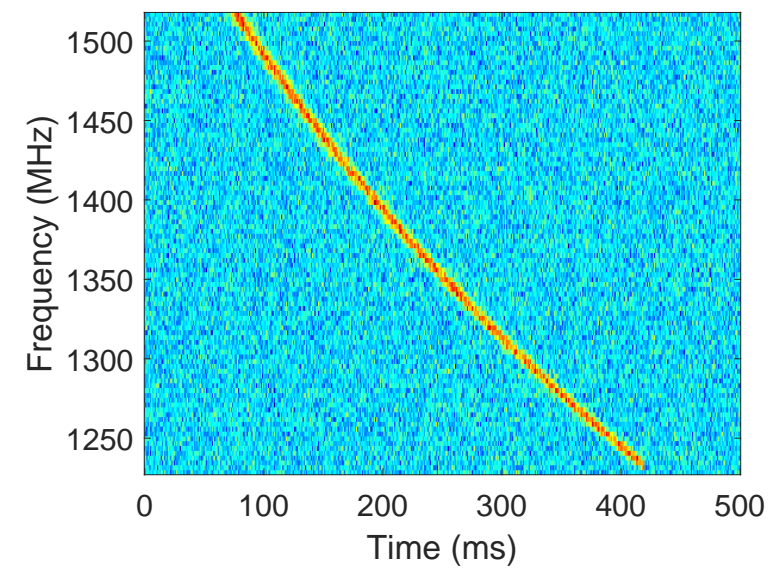

(a) a single pulse

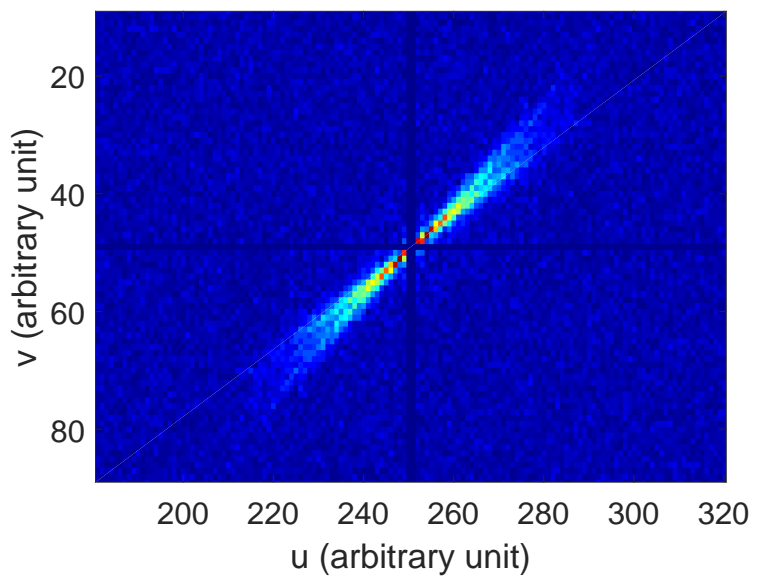

(c) SFT of (a)

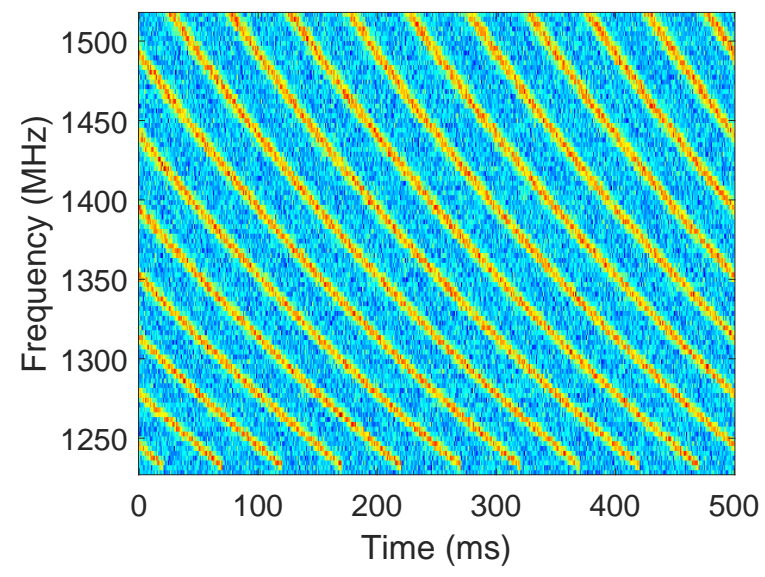

(b) periodic pulse

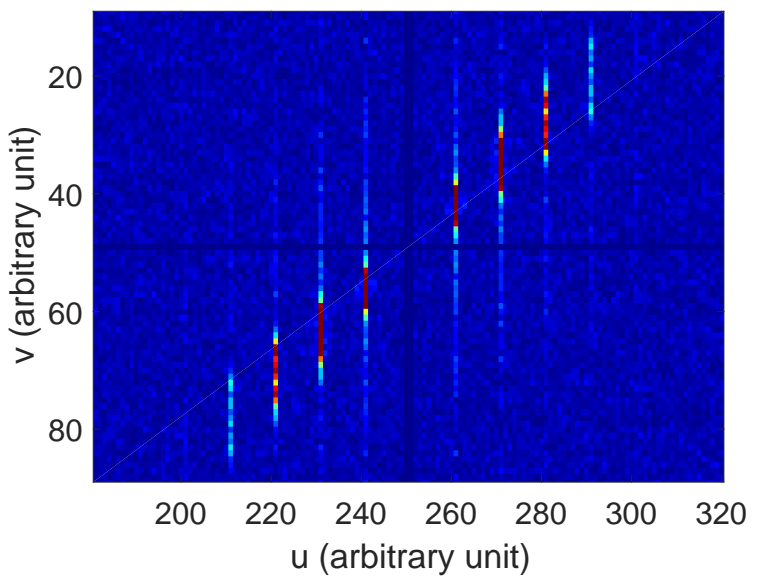

(d) SFT of (b)

Figure 3.1: Shown are an isolated pulse and a periodic signal in filter bank and SFT formats.

The main parameters of the pulse in Fig. 3.1(c) include the pulse width $T$ assuming a rectangular pulse profile, DM value affecting the sweep of the pulse across frequency channels, and Signal-to-Noise (SNR) value of the pulse as determined by the conventional pulse detection algorithm [20]. Let $f_{l o}$ and $f_{h i}$ be the low and high frequencies of the receiver bandwidth, $t_{1}$ and $t_{2}$ be the initial and the final temporal values describing a window in the filter bank format, and $\Pi\left(\frac{t}{T}, \frac{f}{W}\right)$ be a two-dimensional rectangular pulse defined over an area $T \times W$. Then the pulse in Fig. 3.1(a), call it $\gamma(t, f)$, is described mathematically as

$$
\gamma(t, f)=\Pi\left(\frac{t}{T}, \frac{f}{W}\right) * * \delta\left(t_{l o}-t-d\left(\frac{1}{f_{l o}^{2}}-\frac{1}{f^{2}}\right)\right),
$$

where $\delta(\cdot)$ is the Dirac delta function, $t_{l o}$ is the time of signal arrival to the lowest frequency of the receiver band, $d=3.149 \times 10^{3} \times \mathrm{DM}$, DM is in $p c / \mathrm{cm}^{3}$ and $* *$ indicates a two-dimensional 
convolution. The SFT of the pulse, denote it as $\Gamma(u, v)$, is the following product:

$$
\Gamma(u, v)=T W \operatorname{sinc}(T u) \operatorname{sinc}(W v) A(u, v)
$$

where

$$
A(u, v)=\int_{t_{1}}^{t_{2}} \int_{f_{l o}}^{f_{h i}} \delta\left(t_{l o}-t-d\left(\frac{1}{f_{l o}^{2}}-\frac{1}{f^{2}}\right)\right) \exp \{-j 2 \pi(t u+f v)\} d t d f
$$

Here $u$ and $v$ are spatial frequencies, reciprocals of $t$ and $f$.

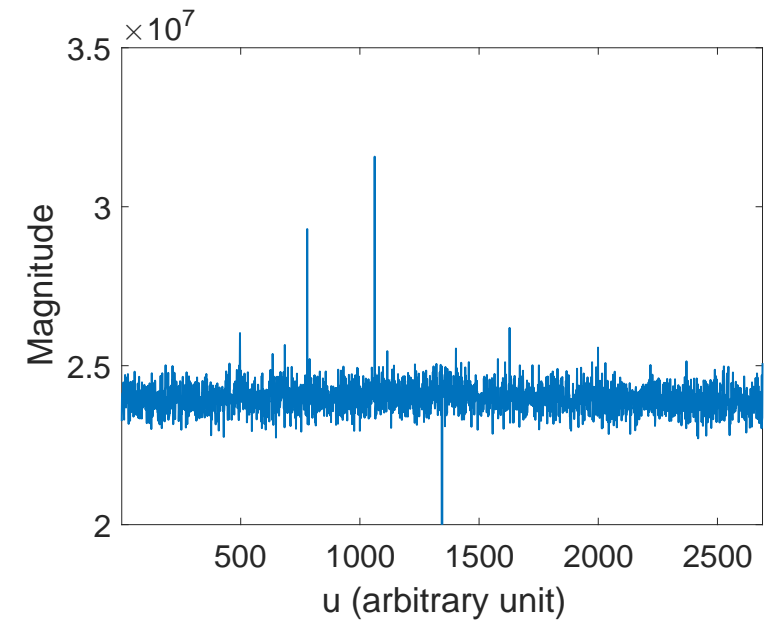

(a) periodic pulse

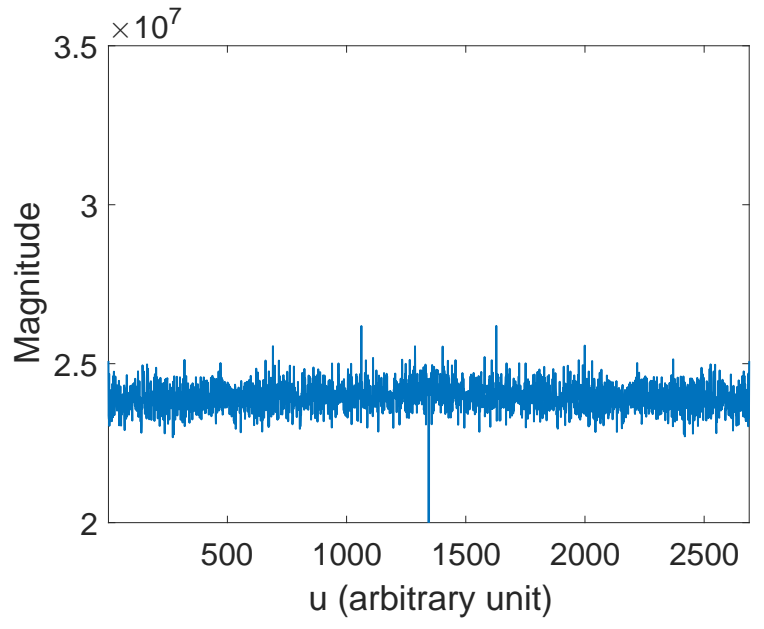

(b) noise + RFI

Figure 3.2: SFT signature of a chunk of PSR B1937+21 integrated over $v$ in the third and forth quadrants of the SFT space.

After replacing the delta function in 3.1 with its periodic version

$$
\sum_{k=-\infty}^{\infty} \delta\left(t_{l o}-t-k T_{0}-d\left(\frac{1}{f_{l o}^{2}}-\frac{1}{f^{2}}\right)\right)
$$

where $T_{0}$ is the rotational period of the periodic astrophysical signal we obtain the following expression for SFT of the periodic pulse in Figure 3.1(b):

$$
\Gamma(u, v)=\frac{T W}{T_{0}} \sum_{k=-\infty}^{\infty} \operatorname{sinc}\left(\frac{T k}{T_{0}}\right) \operatorname{sinc}(W v) A\left(\frac{k}{T_{0}}, v\right) .
$$

As discussed in [40] $A(u, v)$ can be simplified by using first or second order approximations to the integral. In this work we will adopt a basic linear approximation where the dispersion equation is replaced by its linear approximation:

$$
t_{l o}-t=\tilde{d}\left(f-f_{l o}\right)
$$


with $\tilde{d}=d\left(\frac{f_{l o}+f_{h i}}{f_{l o}^{2} f_{h i}^{2}}\right)$. After substituting 3.5 into 3.3 we obtain:

$$
\begin{aligned}
& \int_{t_{1}}^{t_{2}} \int_{f_{l o}}^{f_{h i}} \delta\left(t_{l o}-t-\tilde{d}\left(f-f_{l o}\right)\right) \exp (-j 2 \pi(t u+f v)) d t d f \\
& =\exp \left(-j 2 \pi u\left(t_{l o}+\tilde{d} f_{l o}\right)-j 2 \pi(v-\tilde{d} u)\left(\frac{f_{l o}+f_{h i}}{2}\right)\right) \times \operatorname{sinc}\left((v-\tilde{d} u)\left(f_{h i}-f_{l o}\right)\right) .
\end{aligned}
$$

as a final step, $u$ has to be replaced with $k / T_{0}$.

A periodic astrophysical pulse can be detected by analyzing the magnitude of SFT of data. Denote the data as $X(u, v)$ and define the point $(N / 2, K / 2)$ as its center. The magnitude of $X(u, v)$ integrated over $v$ in the third and forth quadrants results in Fig. 3.2(a) for the case when the data contain periodic pulses, and in Fig. 3.2(b) for the case when no pulse (noise only case) is present in the data. In Fig. 3.2(a), the signature of the periodic pulse appears as a set of relatively strong spikes (sharp peaks) to the left of the center of integration (if the integration is performed in the first and second quadrants, the periodic pulse signature would appear to the right of $N / 2$ ). In Fig. 3.2(b), the result of integration is nearly symmetric around the center. These observations lead to the following decision rule:

$$
\max _{\forall u<N / 2} \sum_{v=1}^{K / 2}|X(u, v)|>\tau \max _{\forall u>N / 2} \sum_{v=1}^{K / 2}|X(u, v)|,
$$

where $\tau>1$ is a decision threshold. The decision rule compares the peak value of the lower half magnitude SFT image integrated over $v$ to the right and left of the center. Pulse is detected if the above inequality holds and not detected, otherwise.

\subsection{Estimation of Rotation}

A few useful signatures of a periodic pulse can be obtained by manipulating data in the SFT space. Integrating the magnitude of SFT of PSR B1937+21 real data over $v$ leads to a representation shown in Fig. 3.3(b). The rotation of periodic astrophysical signals can be estimated from finding the peak locations of the magnitude SFT image integrated over $v$. As demonstrated in Fig. 3.3(b), the peak value occurs at $u_{0}=1628$, where $u$ is measured in bins (dimensionless units). Let $N$ be the number of bins along u-axis and $T_{s}$ be the sampling 


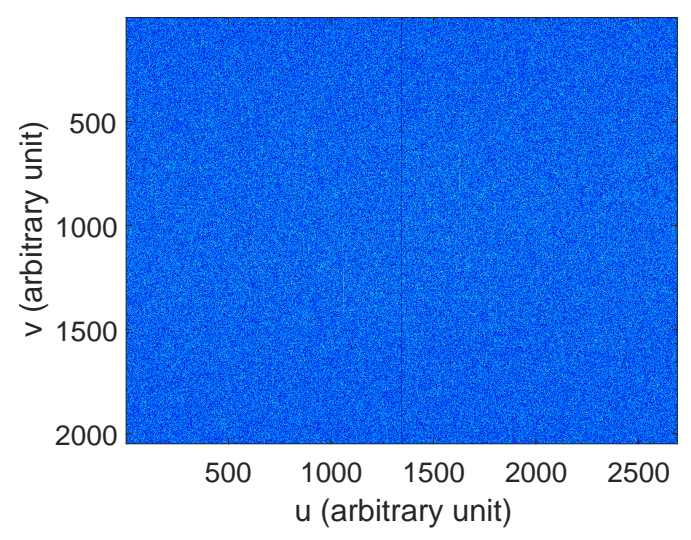

(a) Magnitude SFT of a chunk of PSR B1937+21

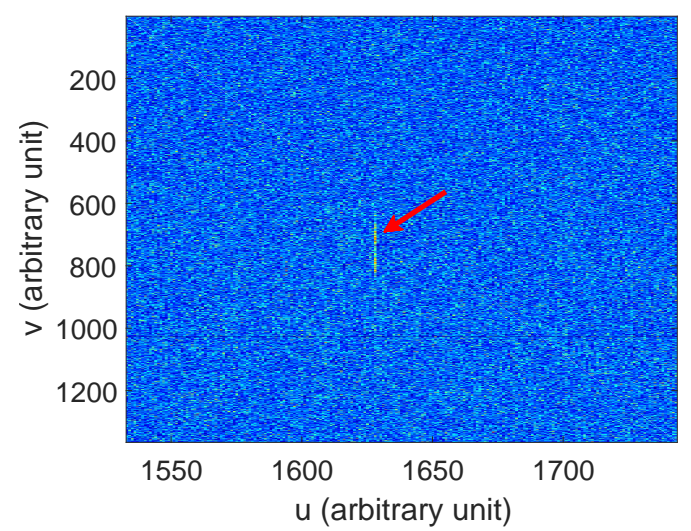

(c) Zoom of magnitude SFT

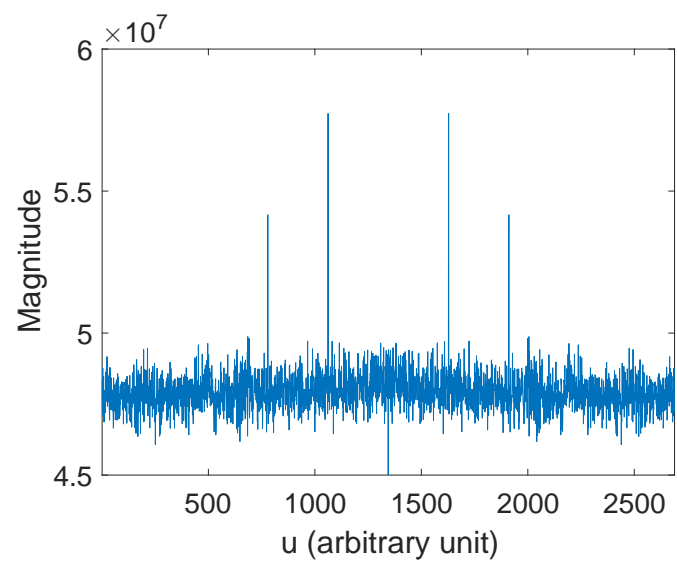

(b) Sum of power over $v$

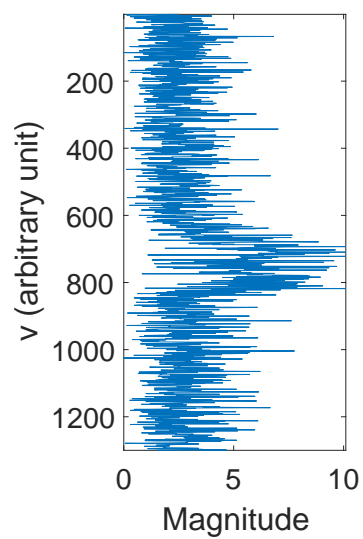

(d) Slice of (c) at $u_{0}=1628$

Figure 3.3: Signature of PSR B1937+21 in the SFT space.

interval (for example, $T_{s}=81.92 \mu s$ for the Green Bank Telescope [19]), then the location of the peak value is related to the rotational period $R$ as follows $u_{0}=N / 2+2 T_{s} N / R$.

For PSR B1937+21 shown in Fig. 3.3, $u_{0}=1628$ pixels and $N=2689$, then

$$
R=\frac{2 N T_{S}}{\left(u_{0}-N / 2\right)}=\frac{2 \times 2689 \times 81.92 \times 10^{-3}}{1628-2689 / 2}=1.55 \mathrm{~ms}
$$

which is matching the true reported value 41 .

\subsection{DM Estimation}

Once $u_{0}$ is found (see the section above), a vertical "slice" of the magnitude SFT at $u_{0}$ (as illustrated in Fig. 3.3 (b) and (c)) can be used to estimate the DM value of the periodic signal 
as well as bounds on it. A lower and upper bounds on the range of estimated DM values of a pulse can be developed as follows. Rewrite the dispersion equation in a linearized form, one equation involving the derivative of the dispersion equation at the highest frequency of the receiver band and the other at the lowest frequency:

$$
f_{h i}-f=\tan (\alpha)\left(t-t_{h i}\right)
$$

and

$$
f-f_{l o}=\tan (\beta)\left(t_{l o}-t\right),
$$

where $\tan (\alpha)<0$ and $\tan (\beta)<0$ are tangents to the dispersion line at the highest and lowest frequencies of the receiver band. The two dispersion equations transformed to the SFT space are given as $u_{h i}=v_{h i} \tan (\alpha)$ and $u_{l o}=v_{l o} \tan (\beta)$.

Given SFT data of a pulsar, the two tangents can be estimated from the SFT "slice" at the fundamental frequency $u_{0}$ as shown in Fig. 3.3(d). A Gaussian curve can be fitted in the data shown in Fig. 3.3(d). Then the boundary points along $v$-direction of the full width at half maximum (FWHM) of the function can be used to estimate $\tan (\alpha)$ and $\tan (\beta)$. The size of the image in Fig. 3.3(b) is $2689 \times 2048$. The locations of the half maximum are 650 and 851. Then

$$
\tan (\alpha)=\frac{(1628-N / 2) \times \frac{1}{N \times T_{s}}}{(K / 2-650) \times \frac{1}{f_{h i}-f_{l o}}}
$$

and

$$
\tan (\beta)=\frac{(1628-N / 2) \times \frac{1}{N \times T_{s}}}{(K / 2-851) \times \frac{1}{f_{h i}-f_{l o}}} .
$$

From (3.7) and (3.8), the estimates of the lower and upper bounds on the DM value are

$$
\mathrm{DM}_{l o}=\frac{f_{l o}^{2} f_{h i}^{2}}{4.149 \times 10^{3} \tan (\alpha)\left(f_{l o}+f_{h i}\right)}
$$

and

$$
\mathrm{DM}_{h i}=\frac{f_{l o}^{2} f_{h i}^{2}}{4.149 \times 10^{3} \tan (\beta)\left(f_{l o}+f_{h i}\right)} .
$$

Applying (3.11) and (3.12) to the PSR B1937+21 data in Fig. 3.3 results in $\mathrm{DM}_{l o}=$ $43.33 \mathrm{pc} / \mathrm{cm}^{3}$ and $\mathrm{DM}_{h i}=93.68 \mathrm{pc} / \mathrm{cm}^{3}$, where the true $\mathrm{DM}=71.0249 \mathrm{pc} / \mathrm{cm}^{3}$. Fig. 3.4 demonstrates similar results for 100 nonoverlapping windows each of size $2689 \times 2048$ cropped from the data of PSR B1937+21. Note the consistency of values of estimates. 


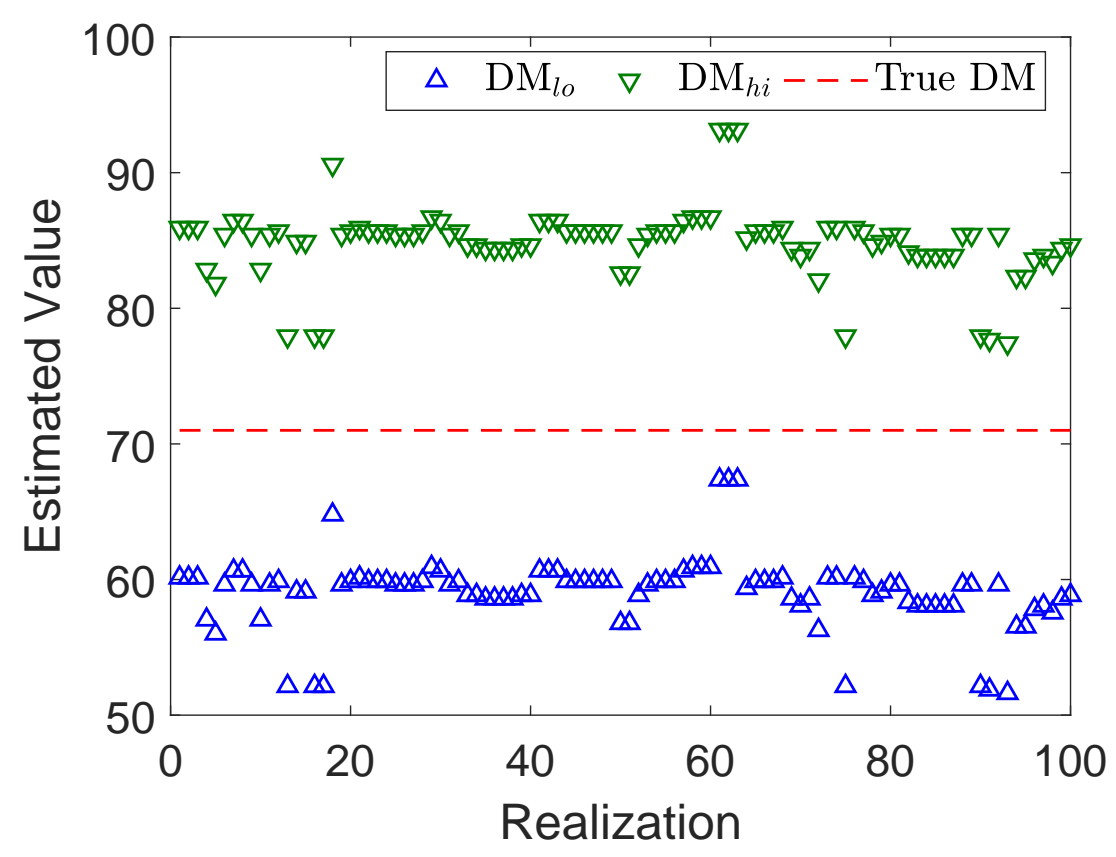

Figure 3.4: Estimation of DM from 100 randomly selected windows of PSR B1937+21.

\subsection{Detection performance}

In this section the performance of the detection rule in (3.6) is numerically analyzed. The performance is evaluated based on simulated data. We assume that the pulse profile is Gaussian with the FWHM set to $10 \mathrm{~ms}$. The period of the simulated pulsar is $100 \mathrm{~ms}$. The bandwidth of the simulated filter bank data is $288 \mathrm{MHz}$ and the center frequency $f_{c}$ is equal to $1374 \mathrm{MHz}$. The bandwidth is partitioned into $\mathrm{K}=96$ discrete frequency channels, and the $\mathrm{DM}$ value is $375 \mathrm{pc} / \mathrm{cm}^{3}$. The time resolution is set to $1 \mathrm{~ms}$. The time duration of generated data is 5 minutes. We use the SNR value of the conventional pulsar detection algorithm to generate realizations of additive noise yielding a correct value of SNR. The conventional SNR is defined as a ratio of the peak value of a de-dispersed and matched-filtered observed signal folded over intervals equal to one period, to the root mean square (RMS) of the simulated noise.

The performance of the detection algorithm is demonstrated through the rate of the true detection at $0 \%$ false alarm rate. Fig. 3.5 displays the detection rate as a function of processed observation time for different values of SNR. Each curve shown in the figure is generated using 1000 Monte Carlo realizations of noisy filter bank data of simulated periodic 


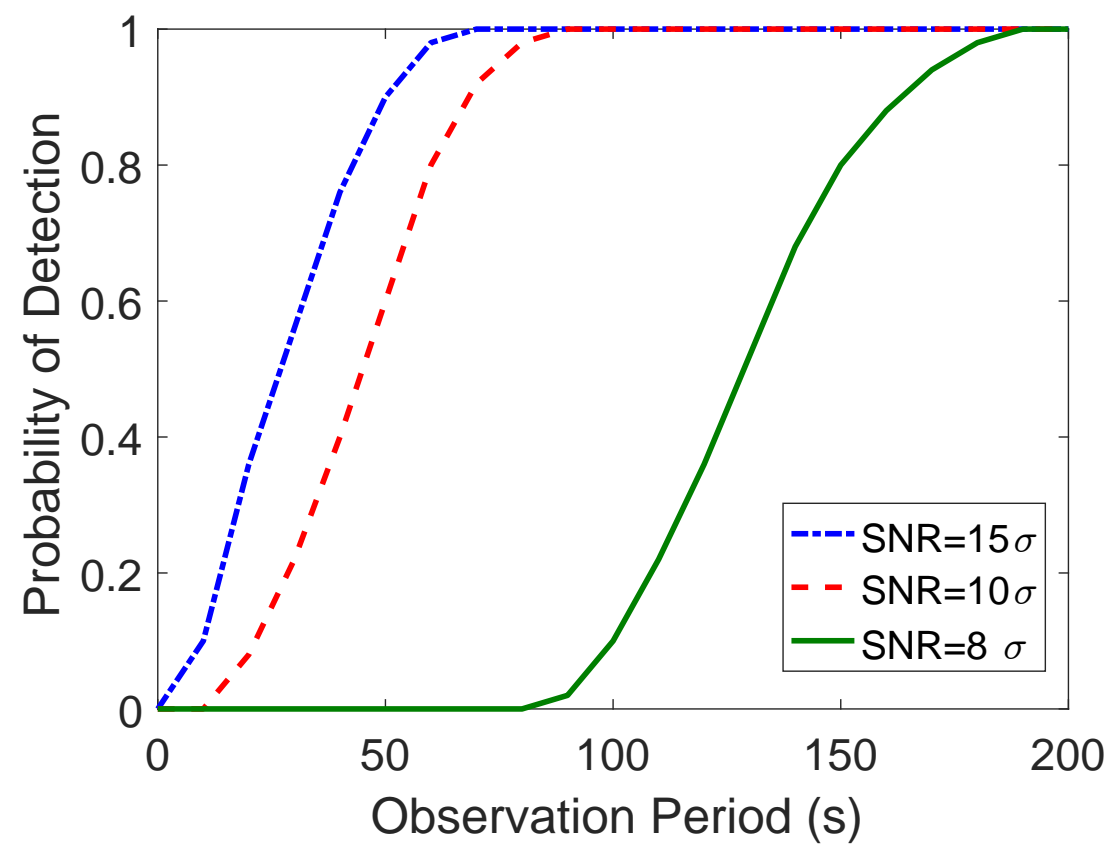

Figure 3.5: Probability of detection as a function of observation time window.

astrophysical pulses. False alarm rate is evaluated using 1000 Monte Carlo simulations of noise.

Note that at $\mathrm{SNR}=8 \sigma$ (the value of SNR is based on a pulse folded over a 5 minute series) the signal is $100 \%$ detectable when the observation time is set to 3 minute. Similarly it takes 80 seconds to reach $100 \%$ detection of the signal parameterized by SNR $=10 \sigma$.

\subsection{Summary}

We introduced and illustrated a pulsar detection based on SFT. Both practical and simulated data were used to illustrate the performance of the developed approaches. The complexity of the detection algorithm is dominated by the complexity of SFT, hence the approach can be used to detect new pulsars in real time. The results of our simulations showed that it takes about 3 minutes of observation time to detect a pulsar signal at an SNR value of $8 \sigma$. Together with the results of detection, the SFT data provide information about the rotation of a pulsar and values of lower and upper bounds on its DM value. 


\section{Chapter 4}

\section{The Effect of Linearized Spectrogram}

\subsection{Introduction}

In this chapter, we expand the algorithm presented in Chapter 2. The algorithm is composed of four main steps. First, a spectrogram is linearized in frequency space. Then it is transformed into a Radon image by means of the Radon transform. The output of the Radon transform is presented as a set of projections parameterized by an angle parameter. Next, the Radon transformed data are smoothed using a spatial low-pass filter. At last the maximum of the transformed data to the right of $90^{\circ}$ is compared to the maximum of the standard deviation of the noise to the left of $90^{\circ}$, and a decision is made in favor of an astronomical pulse to be present or absent using a simple decision rule. Once an astronomical pulse is detected, its DM value is estimated. The performance of the developed detection algorithm and of the DM estimation approach are demonstrated on simulated and experimental data. The results show great promise, at least as well as the conventional algorithm

The remainder of the chapter is organized as follows. Sec. 4.2 provides details of the proposed detection algorithm. Sec. 4.3 describes a simple DM estimation approach, which utilizes the Radon transform of a linearized spectrogram. Sec. 4.5 presents the results of performance analysis. A short summary is provided in Sec. 4.6 . 


\subsection{Detection Algorithm}

The detection algorithm is composed of four consecutive signal processing operations. A block-diagram illustrating them is shown in Fig. 4.1.

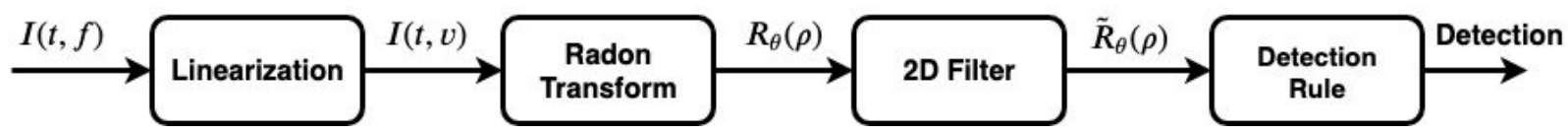

Figure 4.1: A block diagram of the proposed detection approach.

A brief description of each block in the block-diagram is provided below.

\subsubsection{Linearization}

The first block in Fig. 4.1 reshapes the spectrogram of data. Its intention is to transform a dispersed pulse into a linear function of time. Denote by $v$ the reciprocal of squared frequency $1 / f^{2}$. Then the dispersion equation of the linearized pulse is given as

$$
\Delta t(v)=4.15 \times 10^{3} \times \mathrm{DM} \times\left(v-f_{r e f}^{-2}\right),
$$

where DM is the dispersion measure in $\mathrm{pc} / \mathrm{cm}^{3}, f_{\text {ref }}$ is a reference frequency in $\mathrm{MHz}, v=1 / f^{2}$ is in $\mathrm{MHz}^{-2}$, and $\Delta t(v)$ is the dispersion delay in seconds. Fig. 4.2 demonstrates a dispersed pulse as a function of $f$ and of $v$ for the case of $\mathrm{DM}=375 \mathrm{pc} / \mathrm{cm}^{3}$.

Practical spectrograms can be treated as images. The frequency range of a spectrogram is partitioned into discrete channels and the time is expressed in discrete units of time. Consider a spectrogram of size $\mathrm{M}-\mathrm{by}-\mathrm{K}$, where $\mathrm{M}$ is the number of time samples and $\mathrm{K}$ is the number of discrete frequency channels. The frequency bandwidth, BW, is partitioned into equally spaced frequency channels each of size $f_{\text {step }}=\mathrm{BW} / K$. Denote by $f_{l}$ the lowest frequency channel and by $f_{h}$ the highest frequency channel. As indicated earlier, the mathematical description of linearization is given as $v(f)=1 / f^{2}$. This implies that the higest channel in $f$ will be mapped into the lowest channel in $v$. Furthermore, the spacing between two neighboring channels in the transformed space will be determined by the difference between $1 / f_{h-1}^{2}$ and $1 / f_{h}^{2}$, where $f_{h-1}$ is the second highest frequency. Since the spacing between 


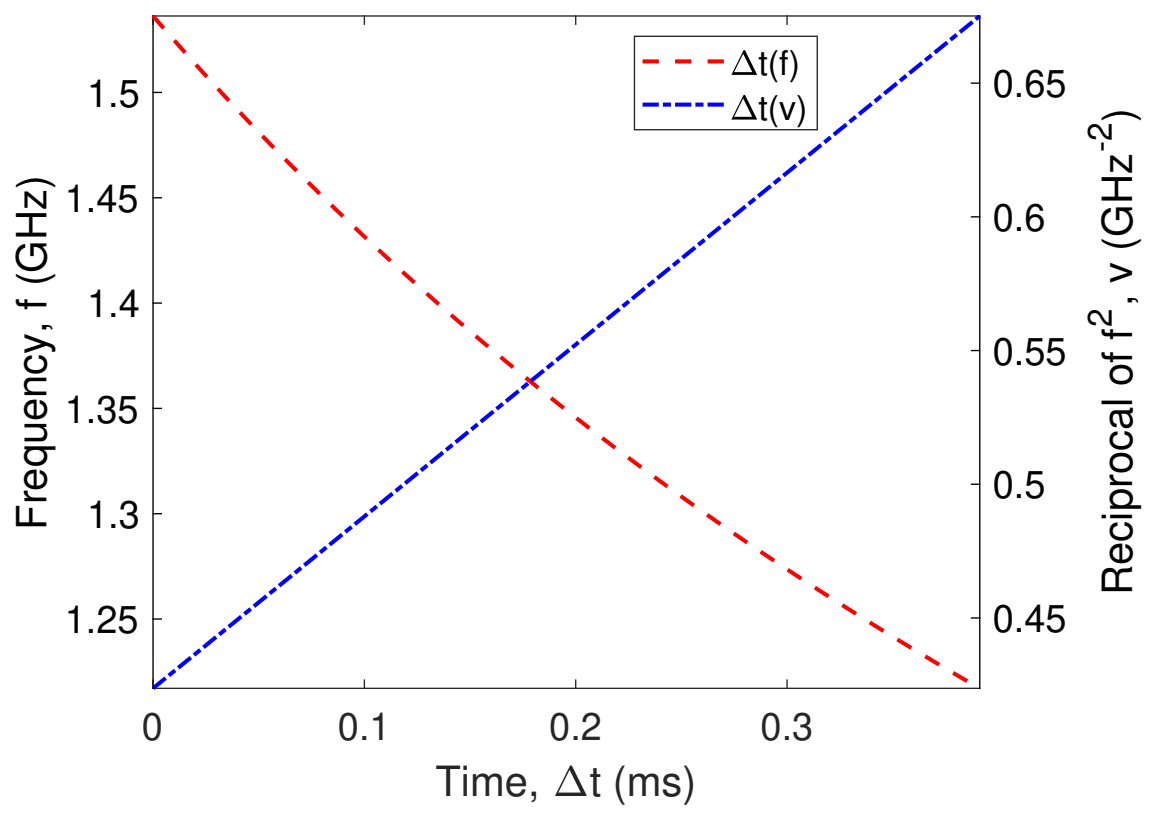

Figure 4.2: Shown on the same plot are a dispersed pulse as a function of time and frequency and its linearized version.

the mapped frequency channels increases with decreasing $f$, not all rows in the linearized spectrogram will have counterparts in the original spectrogram. Some rows will be filled with zeros. The lower the value of $f$, the more rows filled with zeros will be inserted.

To be more specific, linearization includes the following steps.

1. Define $v$ as the reciprocal of squared frequency $\left(v=f^{-2}\right)$, let $\Delta v=1 / f_{h-1}^{2}-1 / f_{h}^{2}$.

2. Map each discrete frequency channel $f$ to a row into a linearized spectrogram. Its position in the linearized spectrogram is determined as $\left(1 / f^{2}-1 / f_{h i}^{2}\right) / \Delta v$.

Algorithm 1 summarizes the linearization steps.

Fig. 4.3 illustrates the linearization. The left panel shows the experimental data of Lorimer burst [3]. The right panel shows its linearized version. Note that the resulting linearized spectrogram has many more rows than the original spectrogram, and many of them are filled with zeros. 
Algorithm 1 Linearization process. Input: $I_{f t}$ is a spectrogram of size M-by-K, $f_{h}$ is the highest in frequency channel, $f_{l}$ is the lowest in frequency channel, and BW denotes the receiver bandwidth. Output: $I_{v t}$ is a linearized spectrogram.
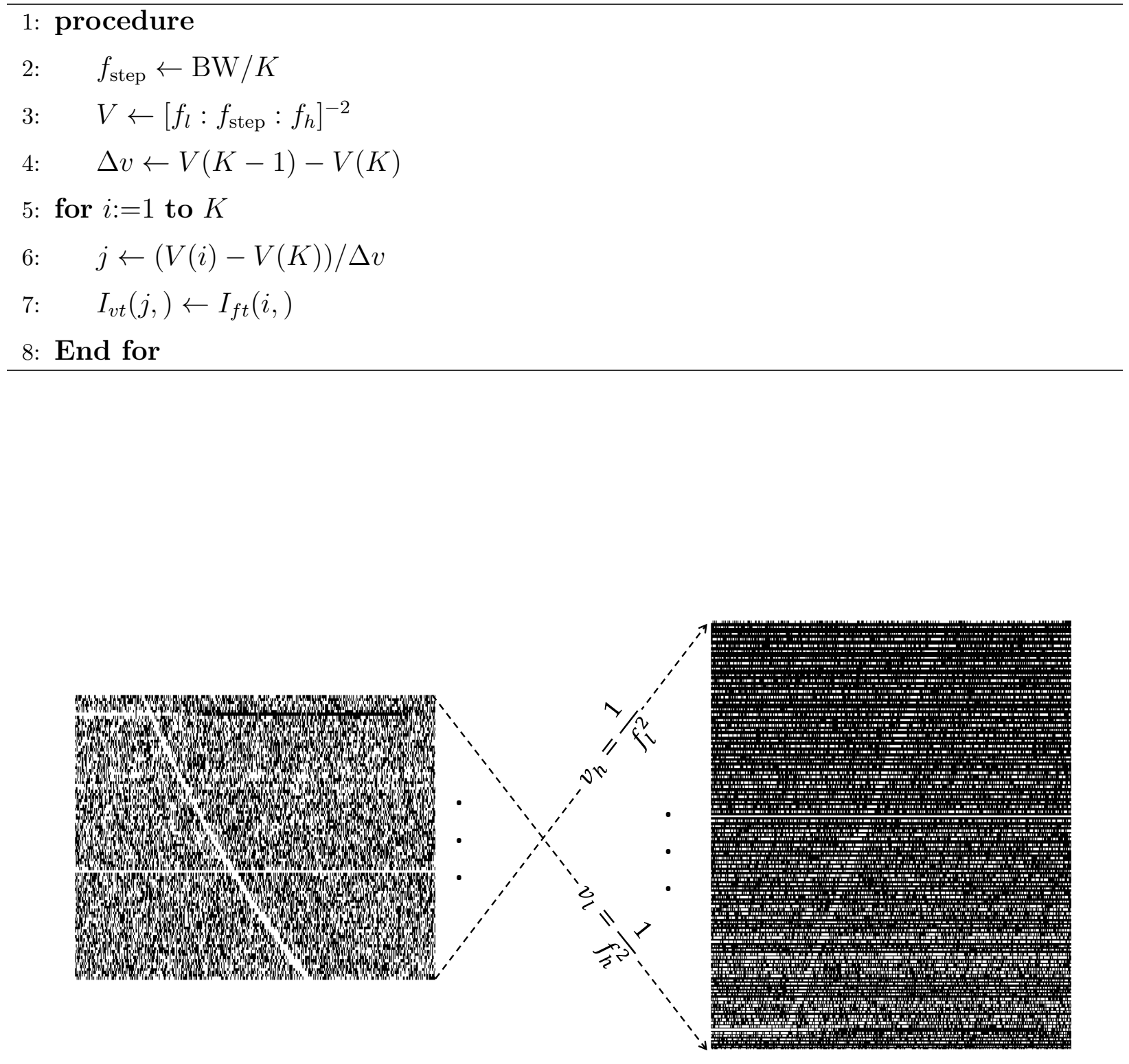

Figure 4.3: Illustration of the linearization. 


\subsubsection{Radon transform}

The second block in Fig. 4.1 applies the Radon transform to a linearized spectrogram. The spectrogram is treated here as an image $I(t, v)$.

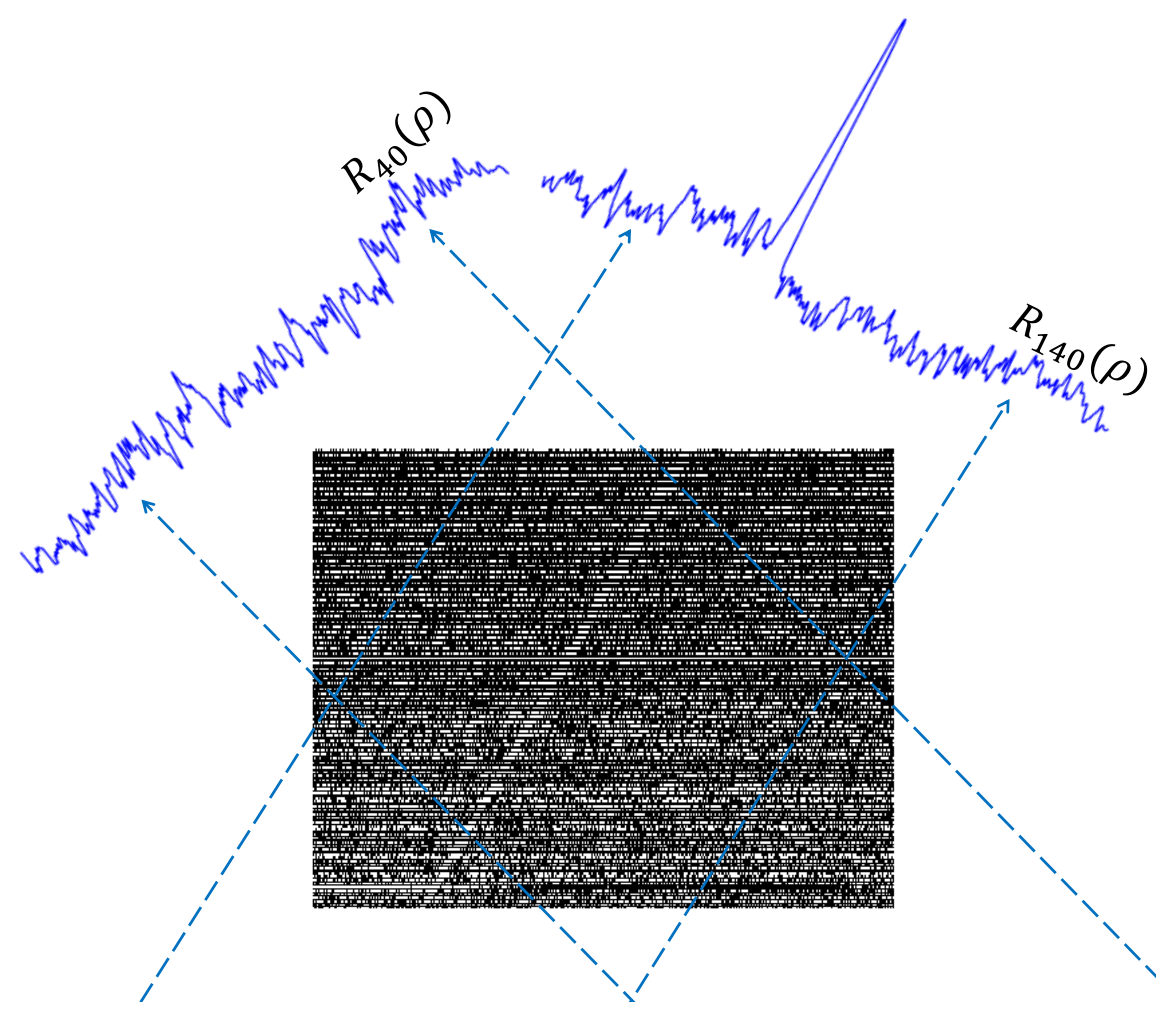

Figure 4.4: The result of applying the Radon transform to the Lorimer burst at two different orientations $\theta=40^{\circ}$ and $\theta=140^{\circ}$.

Mathematically, the Radon transform is defined [37] as

$$
R_{\theta}(\rho)=\int_{t_{i}}^{t_{f}} \int_{v_{l}}^{v_{h}} I(t, v) \delta(\rho-t \cos \theta-v \sin \theta) d t d v,
$$

where $\theta$ is the angle of a projection, $\rho$ is the offset of a projection line from the origin of the spectrogram, $t_{i}, t_{f}, v_{l}$ and $v_{h}$ define four time-frequency boundary points of the spectrogram and $\delta(\cdot)$ is the Dirac delta function. Assuming that an astronomical pulse has a Gaussian profile in both directions, $I(t, v)$ can be expressed as

$$
I(t, v)=h \exp \left(-\frac{t^{2}}{2 \alpha^{2}}-\frac{v^{2}}{2 \beta^{2}}\right) * * \delta\left(t-t_{r e f}-4.15 \times 10^{3} \times \mathrm{DM} \times\left(v-f_{r e f}^{-2}\right)\right),
$$

where $h$ is the peak of the pulse profile in a frequency channel, the parameter $\alpha$ is a spread of the Gaussian bell function in $t$ direction; $\alpha$ is related to the full width at half maximum 


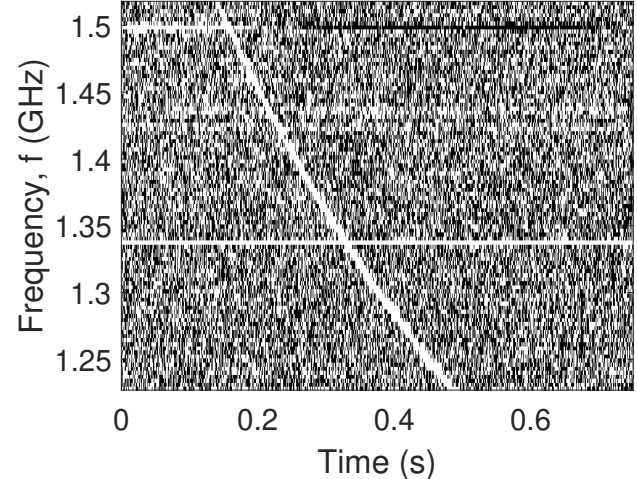

(a) Lorimer burst

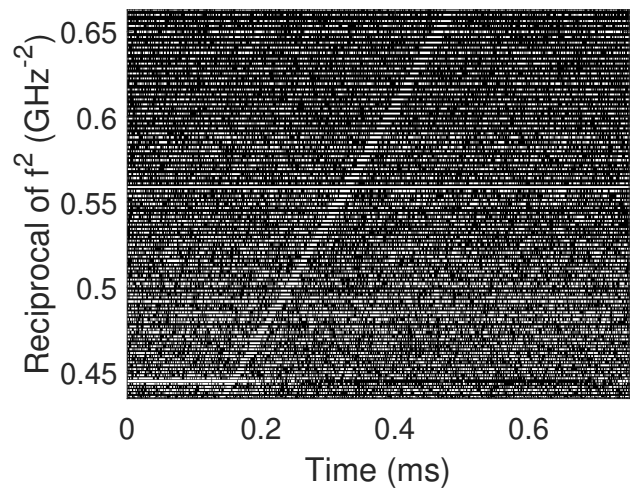

(c) Linearization of (a)

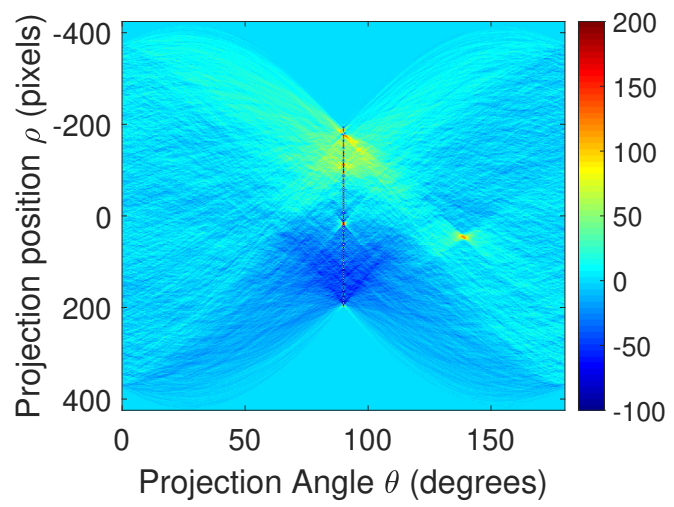

(e) Radon transform of (c)

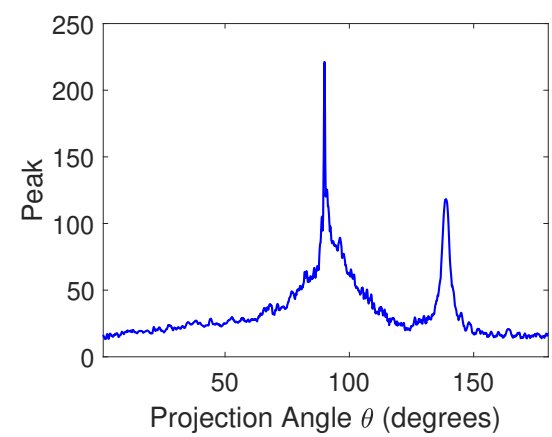

(g) Peak value in (e)

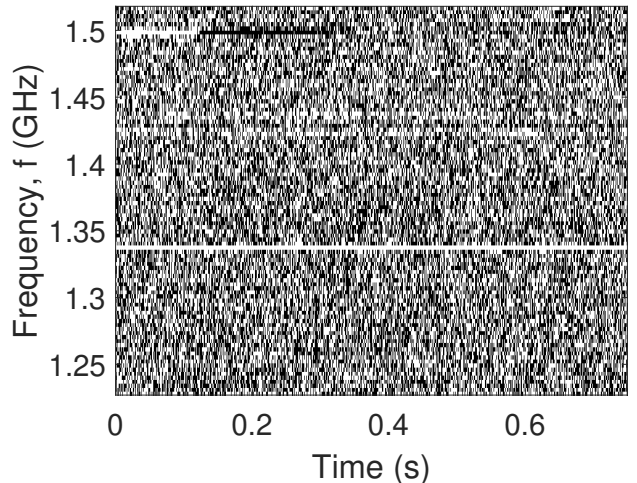

(b) noise + RFI

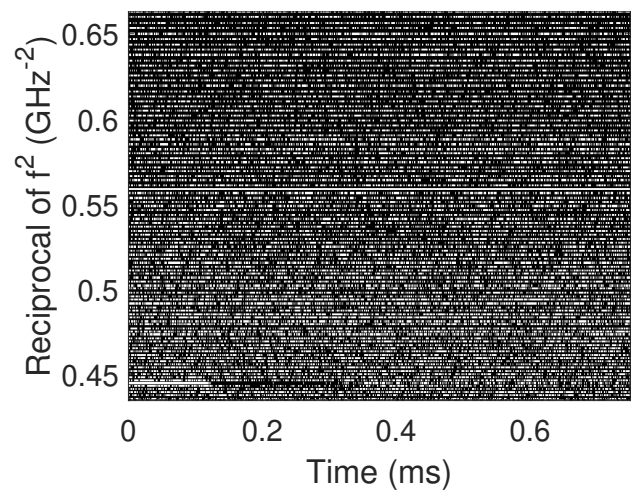

(d) Linearization of (b)

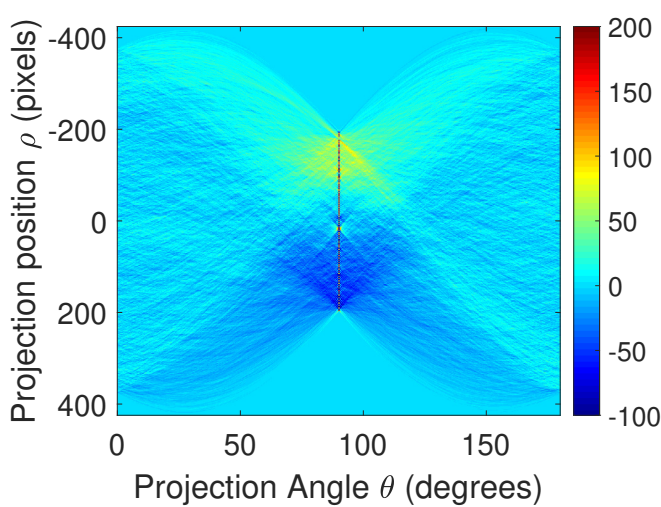

(f) Radon transform of (d)

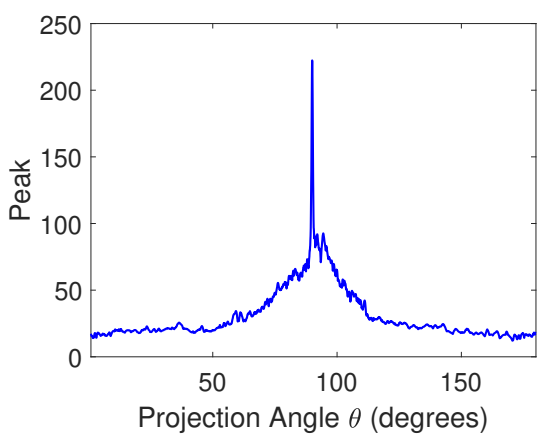

(h) Peak value in (f)

Figure 4.5: Spectrograms of the experimental data of Lorimer burst (shown in panel(a)) and of noise and RFI (shown in panel (b)) and their step wise transformations. 
(FWHM) according to FWHM $=2 \sqrt{2 \ln 2} \alpha$, the parameter $\beta$ is the spread of the Gaussian bell function in $v$ direction, $t_{r e f}$ is the reference time related to $f_{r e f}$, and "**" stands for a two-dimensional convolution. In radio astronomy $f_{r e f}$ is often selected as the central frequency of the receiver bandwidth.

Fig. 4.4 provides an example of applying the Radon transform to the experimental data of linearized Lorimer burst at two different angles $\theta=40^{\circ}$, and $\theta=140^{\circ}$. Fig. 4.5(e) displays the result of the Radon transform as a function of two parameters $\rho$ and $\theta$. The value of the projection at the origin $(\rho=0)$ corresponds to the integral of the pixel values along the line that passes through the middle of the image $I(t, v)$ in Fig. 4.4 , which is also reproduced in Fig. 4.5(c). For comparison, Fig. 4.5(f) displays the Radon transform of the linearized spectrogram shown in panel (d), which displays experimental data of noise plus radio frequency interference (RFI), but no astrophysical pulse. The origin of the Radon transform is at $\left(\rho=0, \theta=90^{\circ}\right)$.

In practice, angles of projections are discretized in the range from 0 to $180^{\circ}$. To save time and reduce complexity of implementation, the discrete projection angles for a linearized spectrogram can be selected as follows

$$
\left\{\theta_{i}=\tan ^{-1}\left(\frac{i}{\tilde{K}}\right): i \text { and } \tilde{K} \text { are integers and } \frac{\Delta t\left(f_{l}, \mathrm{DM}_{\min }\right)}{T_{s}} \leq i \leq \frac{\Delta t\left(f_{l}, \mathrm{DM}_{\max }\right)}{T_{s}}\right\}
$$

where $T_{s}$ is the sampling interval in time, $\tilde{K}$ is the number of rows in the linearized spectrogram, $\Delta t\left(f_{l}, \mathrm{DM}\right)$ is the time delay at the lowest frequency $f_{l}$ for a given DM in the interval from $\mathrm{DM}_{\text {min }}$ to $\mathrm{DM}_{\text {max }}$, which are selected by the user.

\subsubsection{Spatial Smoothing}

To remove spurious peaks attributed to noise as they are unwanted components or features in the data, the Radon transformed data are smoothed with a simple low pass filter of size $5 \times 5$. In our experiments we exploit a Gaussian-shaped filter with the standard deviation parameter set to 1 . Figs. 4.6 and 4.7 show the Radon transformed data before and after smoothing. 


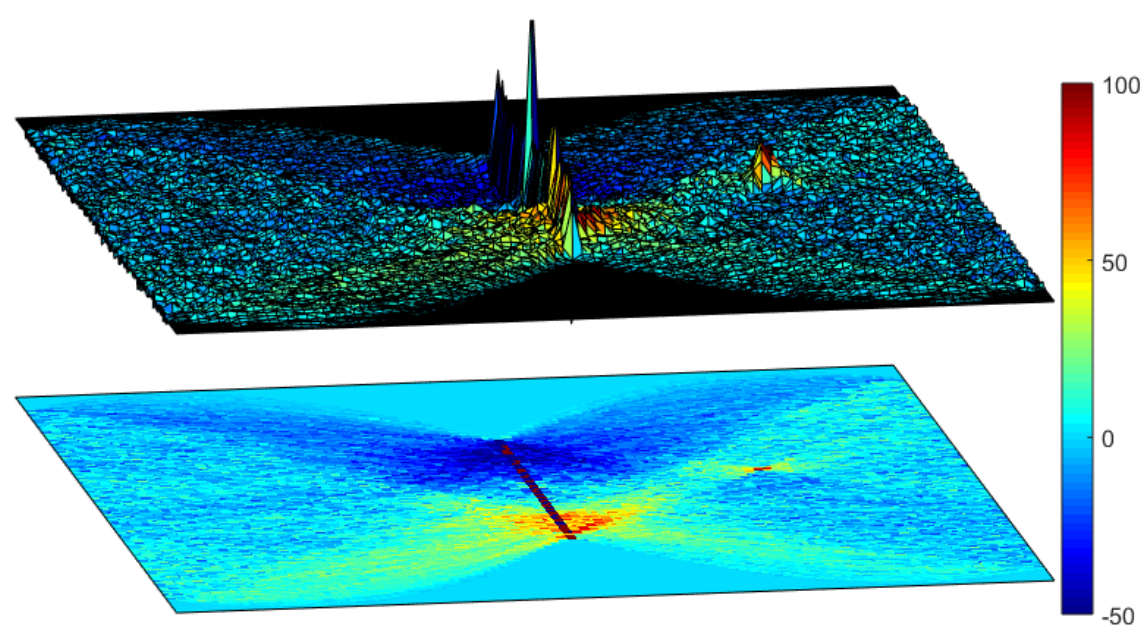

Figure 4.6: Three-dimensional surface plot of Radon image.

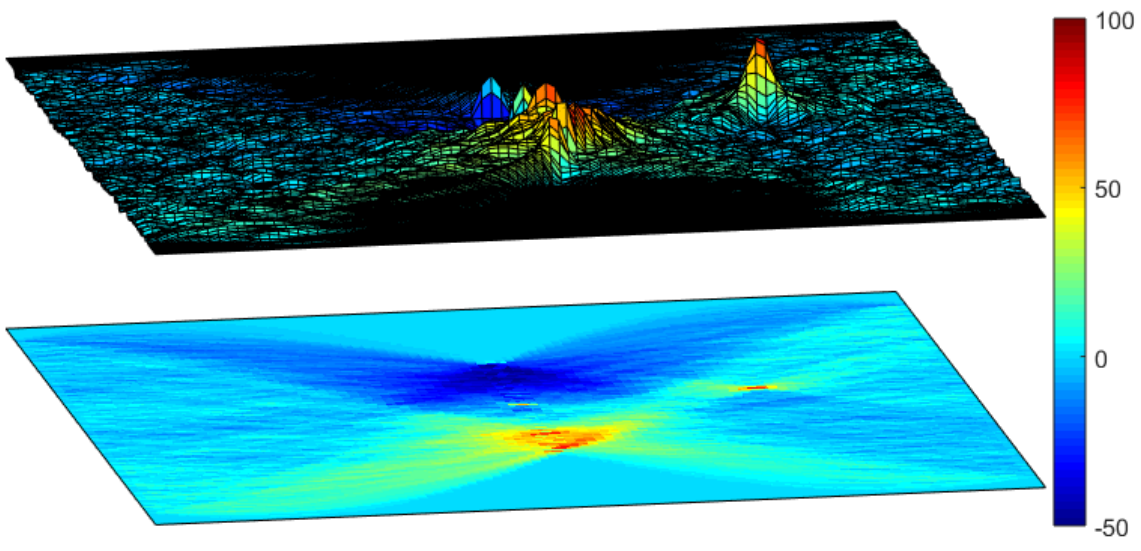

Figure 4.7: Three-dimensional surface plot of filtered Radon image. 


\subsubsection{Decision rule}

In the smoothed data the pulse can be detected by comparing the maximum value of the data to the right of $90^{\circ}$ angle to the standard deviation of the data to the left of $90^{\circ}$ angle. As an illustration, Figs. 4.5 (g,h) show maximal values of smoothed Radon transform data as a function of the parameter $\theta$. Here the maximal value is found along each column, that is, maximum is taken over the values of $\rho$. Note that in the case of pure noise the function is nearly symmetrical around $90^{\circ}$ compared to the case when data contain a pulse; the function shows a peak to the right of $90^{\circ}$ angle.

Let $r_{\theta}$ be the value of the Radon transformed data along the column parameterized by $\theta$, when $\theta \in\left(90^{\circ}+\epsilon, 180^{\circ}-\epsilon\right)$

$$
r_{\theta}=\max _{\rho} R_{\theta}(\rho)
$$

The angle $\theta$ is defined in Eq. 4.4 and $\epsilon$ is a small number introduced to avoid inclusion of RFI. RFI is often constrained to either a narrow band of frequencies (narrow band RFI) or to a short in duration interval in time (bursty RFI). When transformed to the Radon space, the two types of RFI are observed either very close to $\theta=0^{\circ}$ or to $\theta=90^{\circ}$.

Let $\sigma_{\theta}$ be the estimated standard deviation of the data along the column parameterized by $\theta$ with $\theta \in\left(\epsilon, 90^{\circ}-\epsilon\right)$

$$
\sigma_{\theta}=\underset{\rho}{\operatorname{Std}} R_{\theta}(\rho)
$$

Then the decision rule can be mathematically formulated as follows. Decide a candidate pulse is present if

$$
\max _{\theta \in\left(90^{\circ}+\epsilon, 180^{\circ}-\epsilon\right)} r_{\theta}>\gamma \max _{\theta \in\left(\epsilon, 90^{\circ}-\epsilon\right)} \sigma_{\theta} .
$$

where $\gamma$ is a decision threshold $(\gamma>1)$. If Eq. 4.7 is false, then there is no detection. For an almost zero false alarm, $\gamma$ can be set to 5 .

We can also implement an alternative detection approach by drawing analogy with the conventional algorithm [20]. The Radon transformed data can be matched filtered with a collection of local two-dimensional filters (for example, Gaussian shaped filters) of varying width. Then the pulse detection is performed by comparing the value of the peak of the filtered data against the value of the estimated standard deviation of the noise evaluated in 
the neighborhood of the peak. If the purpose of the detection rule is to seek for astrophysical pulses, then the search of pulses need not to extend beyond $\theta \in\left(90^{\circ}+\epsilon, 180^{\circ}-\epsilon\right)$.

\subsection{Estimation of DM Value}

In addition to pulse detection based on the Radon transform of linearized spectrograms, the same data can serve the purpose of DM estimation. After a candidate pulse is detected, we have enough information to estimate its DM value. We can develop a basic equation relating the slope of the linearized dispersed pulse and the DM value. The estimate of the DM value is obtained by analyzing the plot of the maximum value of the Radon transformed data as a function of the projection angle $\theta$ (see Fig. 4.5 (g)). The signature of the pulse in Fig. 4.5(e) can be approximated by a narrow Gaussian curve with $\theta_{\text {peak }}$ as the location of the peak value of the curve. This angle is used to find the estimated DM value. Note that in the linearized spectrogram of a pulse, $\theta_{\text {peak }}$ can be obtained from the slope of the dispersed linearized pulse. The equation of the line is given as

$$
\left(t-t_{h}\right) \tan \left(\theta_{\text {peak }}-90\right)=v-v_{l}=f^{-2}-f_{h}^{-2}
$$

interchangeably with

$$
\left(t_{l}-t\right) \tan \left(\theta_{\text {peak }}-90\right)=v_{h}-v=f_{l}^{-2}-f^{-2}
$$

where $t_{l}$ and $t_{h}$ are the time instances of the pulse arrival to the lowest $f_{l}$ and the highest $f_{h}$ frequencies of the receiver band, $\tan \left(180-\theta_{\text {peak }}\right)$ is the tangent of the dispersion line.

Setting $f$ in Eq. 4.8 to $f_{l}$ and $t$ to $t\left(f_{l}\right)$, then dividing it by the dispersion equation below

$$
t\left(f_{l}\right)-t_{h}=\mathrm{DM} \times 4.15 \times 10^{3} \times\left(f_{l}^{-2}-f_{h}^{-2}\right) .
$$

results in the following DM estimate:

$$
\widehat{\mathrm{DM}}=\frac{1}{\tan \left(\theta_{\text {peak }}-90\right) \times 4.15 \times 10^{3}} .
$$

If a spectrogram is treated as an image (discrete in time and frequency), $\theta_{\text {peak }}$ is expressed in terms of the sampling interval $T_{s}$ and the number of discrete frequency channels in the 
linearized spectrogram $\tilde{K}$ resulting in

$$
\widehat{\mathrm{DM}}\left(N, T_{s}\right)=\frac{N T_{s}}{\tan \left(\theta_{\text {peak }}-90\right) \times 4.15 \times 10^{3} \times\left(f_{l}^{-2}-f_{h}^{-2}\right)} .
$$

\subsection{Detection of Periodic Astrophysical Pulses}

The algorithms proposed in the previous sections can be easily extended such that it can be applied to detect periodic pulses. The primary difference is in the detection rule. Fig. 4.8 shows the main steps of the detection algorithm applied to a periodic signal. For more detailed description and analysis of periodic pulses refer to Sec 1.3 .

Fig. 4.8(a) shows the spectrogram of a simulated millisecond pulsar. Its SNR is intentionally set to a high value to clearly illustrate each step of the detection algorithm and how it affects the astronomical signal. Fig. 4.8(b) shows the linearized specrogram, the Radon transform of the linearized specrogram is shown in Fig. 4.8(c). The pulsar can be detected by applying the FFT across the projection axis at each angle. The projection in Fig. 4.8(d) then folded at the highest detected harmonic to increase the signal to noise ratio as in Fig. 4.8(f). The DM of the periodic pulses can be estimated using Eq. 4.12, The period of the simulated pulsar can be found as the reciprocal of the fundamental frequency found as a distance between two adjacent peaks.

\subsection{Performance Analysis}

In this section we evaluate the performance of the detection and DM estimation algorithms. The performance of the detection algorithm is demonstrated by means of Detection Rate and False Alarm Rate 38]. Due to a very limited amount of data containing real FRB pulses, our numerical evaluation involving FRBs mostly relies on simulated data. RRAT series used in this evaluation are provided by the Department of Physics and Astronomy at West Virginia University [12]. 


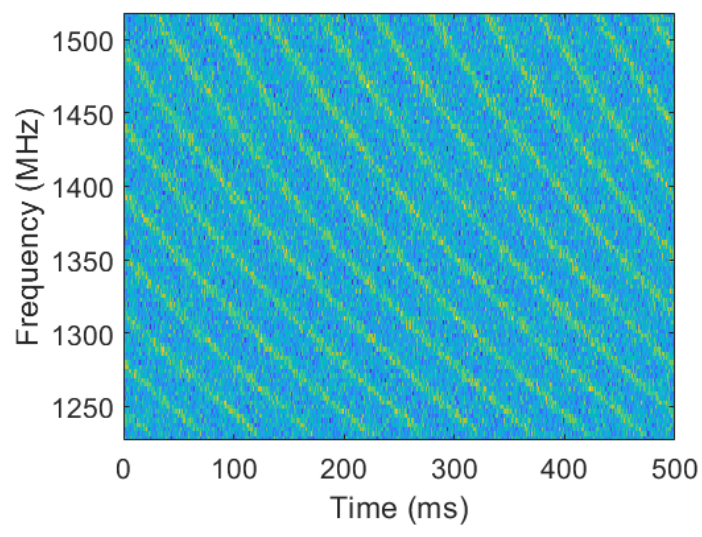

(a) Periodic Pulses

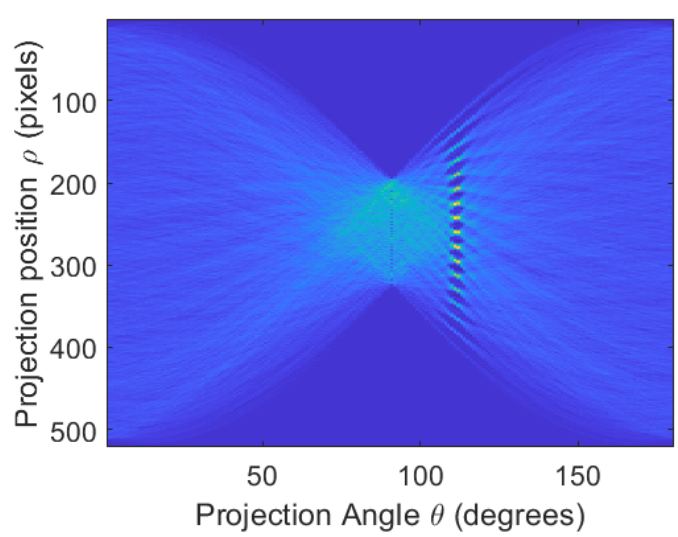

(c) The Radon transform of (b)

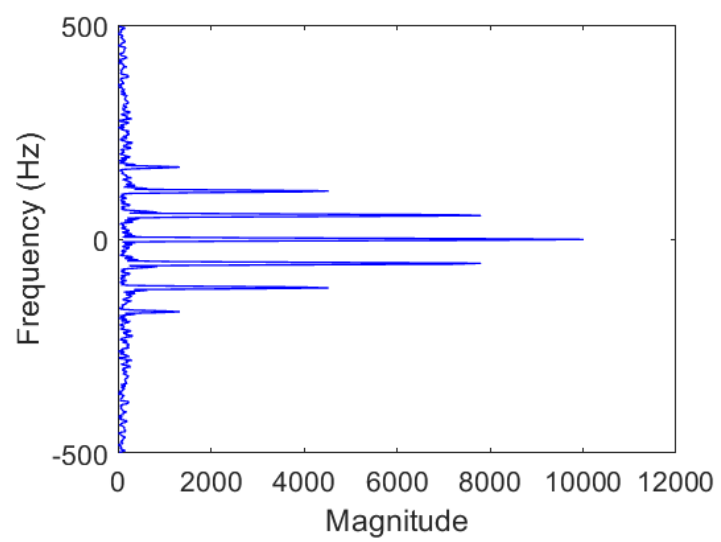

(e) FFT of (d)

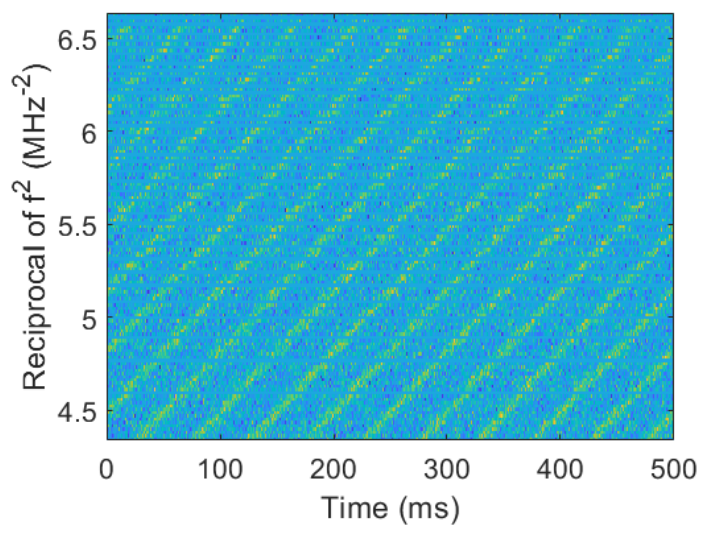

(b) Linearized pulses

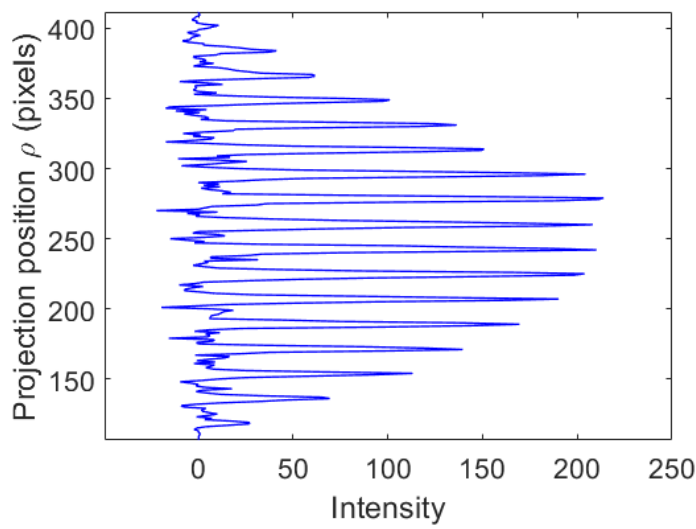

(d) Projection at $\theta=120^{\circ}$

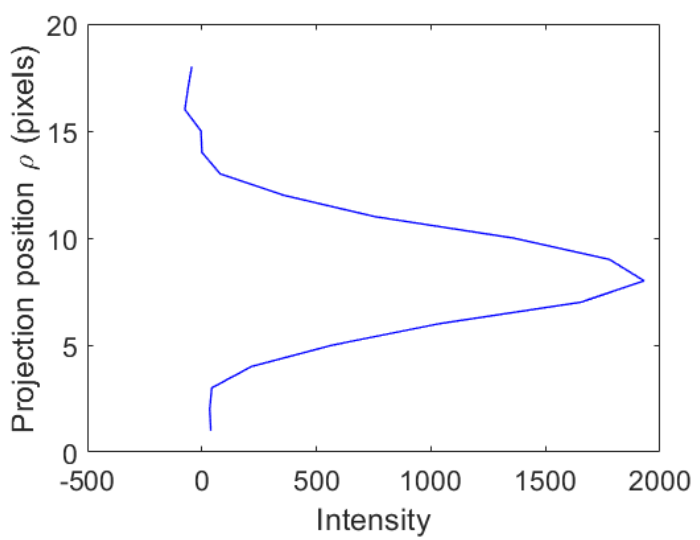

(f) Folded pulses from (d)

Figure 4.8: Spectrogram of a simulated millisecond pulsar shown in panel (a) and its step wise transformations. 


\subsubsection{Numerical Evaluation based on Simulated Data}

The initial data are in the form of spectrograms. We assume that the pulse profile of each astronomical pulse is Gaussian with the FWHM set to correspond to the width of a pulse of interest. The bandwidth of a simulated spectrogram, the sampling interval, the number of discrete frequency channels, the SNR of the pulse, and the DM value of a simulated pulse are set to the values provided in Petroff et al. [5]. To add noise to a simulated astronomical pulse, we apply the conventional definition of the SNR, where it is defined as a ratio of the peak value of a de-dispersed and matched-filtered observed signal to the root mean square (RMS) of the simulated noise. Details of our simulation approach are provided in Schmid et al. 30 .

\section{Pulse Detection}

To analyze the detection performance of the developed algorithm we simulate 10,000 instances of each FRB listed in Table 4.1. For each FRB we also generate 10,000 instances of pure noise. The decision rule in Eq. 4.7 is applied to both noisy data containing a pulse and to data containing pure noise. The value of parameter $\gamma$ is selected such that the estimated False Alarm Rate (based on 10,000 instances of pure noise) is zero. The last column in Table 4.1 presents the Detection Rate for each analyzed FRB. To demonstrate the robustness of our algorithm to noise, we lower the SNR value to 8 and repeat the previous experiment. Note that the remaining simulation parameters such as number of channels, sampling time, bandwidth, and other parameters of the telescopes are kept unchanged, that is, they are set to those listed in Petroff et al. [5]. The numerical evaluation shows that the algorithm generates no False Alarms and detects every simulated pulse $(100 \%$ detection rate, as before).

\section{The effect of pulse width on the detection performance}

The results of the previous subsection demonstrate that all simulated pulses detectable by the conventional algorithm are also detectable by the developed algorithm. In this subsection we numerically analyze the effect of the pulse width on the detection performance. Our claim 
Table 4.1: A list of fast radio bursts used in our numerical analysis.

\begin{tabular}{|c|c|c|c|c|c|c|}
\hline Name & Telescope & $\begin{array}{c}\text { DM } \\
\left(\mathrm{pc} / \mathrm{cm}^{3}\right)\end{array}$ & $\begin{array}{c}\text { Width } \\
(\mathrm{ms})\end{array}$ & $\begin{array}{c}\text { Detected } \\
\text { SNR }\end{array}$ & $\begin{array}{c}\text { Tested } \\
\text { SNR }\end{array}$ & $\begin{array}{c}\text { Detection } \\
\text { Rate }\end{array}$ \\
\hline \hline FRB121102 & Arecibo & 557 & 3 & 14 & 8 & $100 \%$ \\
\hline FRB110220 & Parkes & 944.38 & 5.6 & 49 & 8 & $100 \%$ \\
\hline FRB160608 & UTMOST & 682 & 9 & 12 & 8 & $100 \%$ \\
\hline FRB170107 & ASKAP & 609 & 2.4 & 16 & 8 & $100 \%$ \\
\hline FRB180301 & Parkes & 522 & 2.18 & 16 & 8 & $100 \%$ \\
\hline FRB180311 & Parkes & 1575.6 & 12 & 11.5 & 8 & $100 \%$ \\
\hline FRB170922 & UTMOST & 1111 & 26 & 22 & 8 & $100 \%$ \\
\hline FRB110626 & Parkes & 723 & 1.4 & 11 & 8 & $100 \%$ \\
\hline FRB170107 & ASKAP & 203.1 & 1.6 & 8.1 & 8 & $100 \%$ \\
\hline
\end{tabular}

is that for a given DM and SNR values, the performance of our algorithm is similar to that of the conventional algorithm regardless of the width of the pulse.

To demonstrate this, we simulated 10,000 astronomical pulses for two values of DM, 300 $\mathrm{pc} / \mathrm{cm}^{3}$ and $1500 \mathrm{pc} / \mathrm{cm}^{3}$, and several values of the pulse width. The input SNR in all simulations was set to 8. Fig. 4.9 shows the count of detected pulses, that is the count of the pulses with estimated SNR above 5, as a function of the pulse width for the DM value of $300 \mathrm{pc} / \mathrm{cm}^{3}$ in the left panel and for the DM value of $1500 \mathrm{pc} / \mathrm{cm}^{3}$ in the right panel, respectively.

Although it appears from the bar plots in Fig. 4.9 that the performance of our algorithm is inferior to the performance of the conventional algorithm, especially for narrow pulses with the width below $3 \mathrm{~ms}$, we have to caution the readers that our simulations are set up in favor of the conventional pulse detection approach. The pulses are de-dispersed using the knowledge of the true pulse width and the dispersion curve itself. This results in a perfectly de-dispersed pulse, as if there is an oracle who supplied the ground truth. This is not possible in practice. We claim if we had empirical data to test the performance of our algorithm, it would perform at least as well as the conventional algorithm. To demonstrate this matter we appealed to simulations. When generating a pulse we added the information about 
the reciprocal of squared frequency to the Radon Transform step, hence this granted the transform to integrate pixels with correct rounding. This procedure is not required in practice and we only added it to show that both algorithms have similar detection performance. Fig. 4.10 shows the bar plots when this idea is implemented.

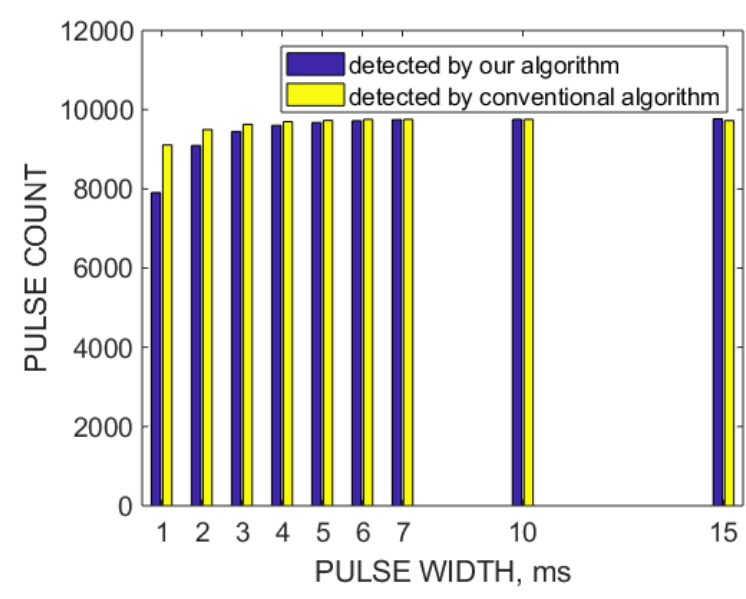

(a) $\mathrm{DM}=300 \mathrm{pc} / \mathrm{cm}^{3}$

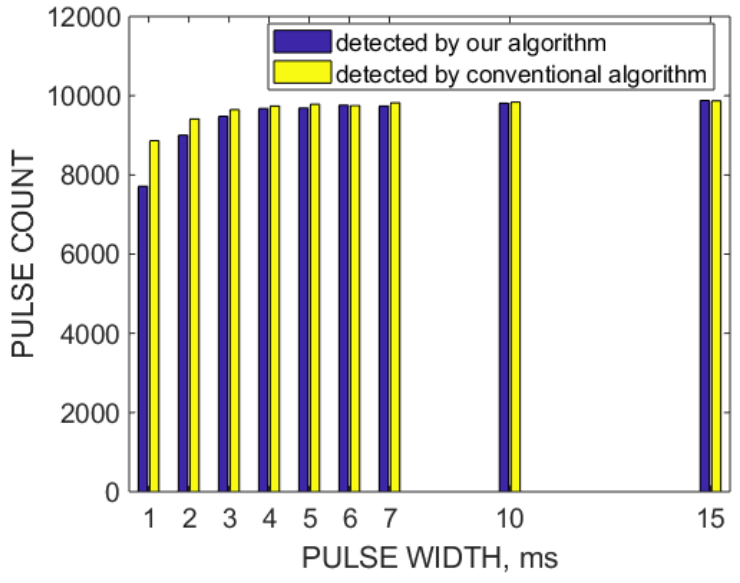

(b) $\mathrm{DM}=1500 \mathrm{pc} / \mathrm{cm}^{3}$

Figure 4.9: Shown are two bar plots displaying the dependence of the number of detected pulses by our and conventional algorithms versus the width of pulses. The input SNR is set to 8 , the number of simulated pulses for each DM is set to 10,000, and the value of DM is set to $300 \mathrm{pc} / \mathrm{cm}^{3}$ in the left panel and to $1500 \mathrm{pc} / \mathrm{cm}^{3}$ in the right panel.

\section{Effects of radio frequency interference}

In this part of the analysis, we aim at evaluating the performance of the newly developed detection algorithm in the presence of RFI. RFI can be placed in two broad categories based on occupied frequencies - narrow band or broadband (for more details about RFI signals refer to Sec 1.2.2). We simulate several scenarios. We first consider the case when RFI corrupts one fifth of the spectrogram data. Fig. 4.11(a) illustrates the case of a narrowband RFI while Fig. 4.11(c) presents an example of a broadband RFI signal. Detection performance of the conventional and our algorithms in application to the corrupted data is illustrated in Fig. 4.11(b) and Fig. 4.11(d), respectively. The degradation in overall performance is due to the fact that RFI corrupts a good portion of data including the region where the pulse is positioned. In Fig. 4.12 we are repeating the same experiments but the RFI corrupts one 


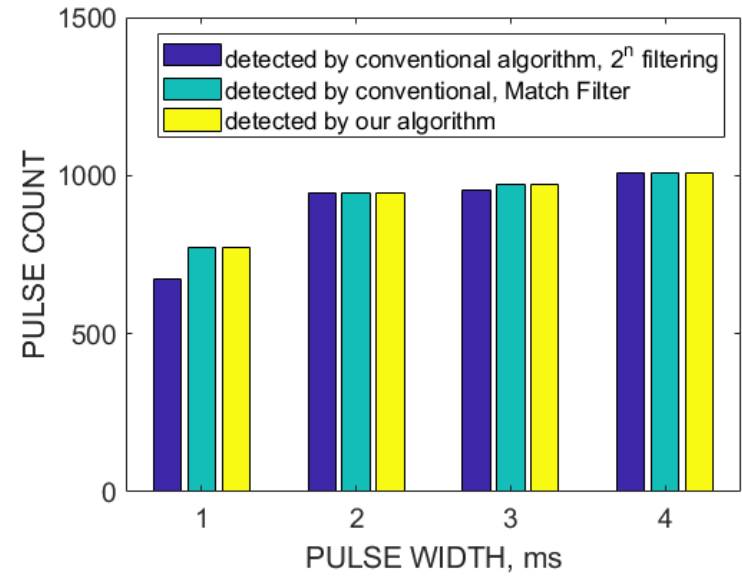

(a) $\mathrm{DM}=300 \mathrm{pc} / \mathrm{cm}^{3}$

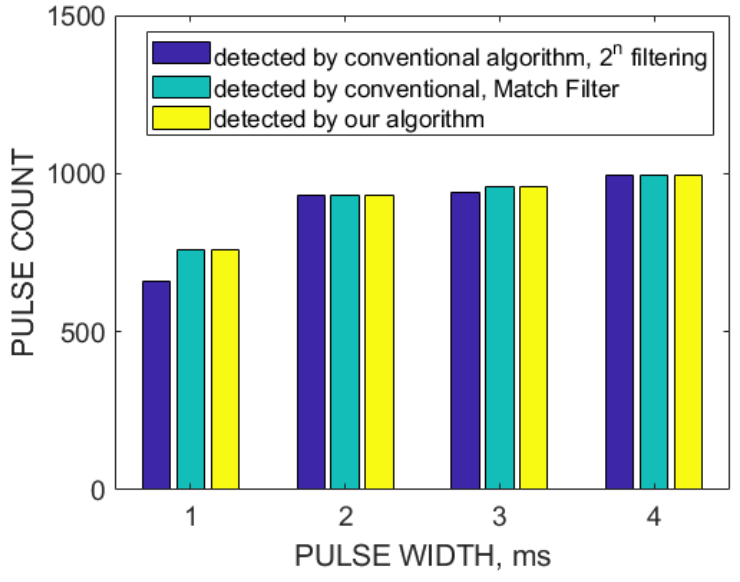

(b) $\mathrm{DM}=1500 \mathrm{pc} / \mathrm{cm}^{3}$

Figure 4.10: Shown are two bar plots displaying the dependence of the number of detected pulses by our and conventional algorithms versus the width of pulses. The input SNR is set to 8 , the number of simulated pulses for each DM is set to 1,000, and the value of DM is set to $300 \mathrm{pc} / \mathrm{cm}^{3}$ in the left panel and to $1500 \mathrm{pc} / \mathrm{cm}^{3}$ in the right panel.

third of the spectrogram data. The figures indicate that the presence of RFI in the data affects the performance of both algorithms in a similar manner.

For the case of periodic pulses, the effect of the pulse width on the detection performance is shown in Fig. 4.13. Experimental setup is similar to the set up described above. The period of the simulated pulsar is equal to $44 \mathrm{~ms}$.

\section{DM Estimation}

We also apply the DM estimation approach outlined in Sec. 4.3 to the simulated data described above. Fig. 4.14 displays six plots of the estimated DM values for each generated instance. The estimated values are shown as blue dots. The dashed horizontal line represents the true DM value of each FRB, the values of the true DM are listed in Petroff et al. [5]. A close inspection of each plot in Fig. 4.14 shows that the estimated DM value for each simulated FRB lies within relatively narrow margins of the true value of DM. The margins are completely dependent on the instrumentation and the characteristics of the data. 


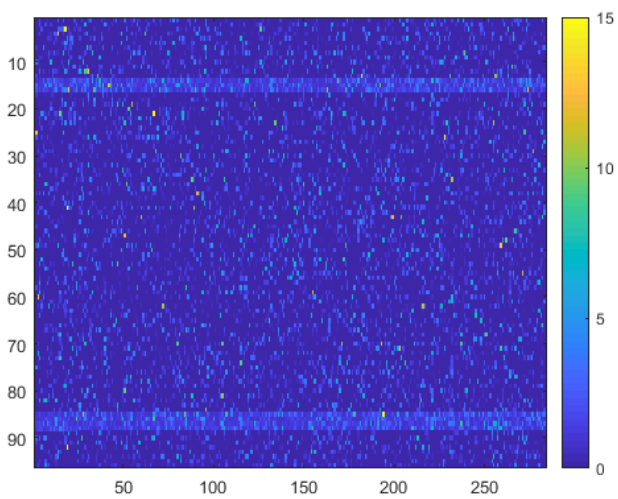

(a) Example of channelized RFI

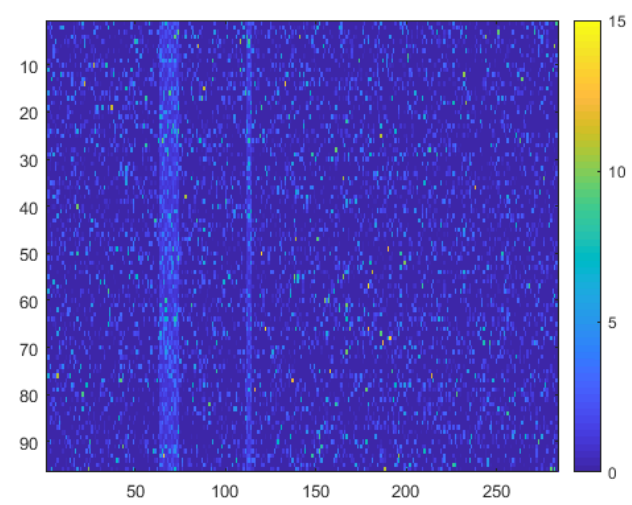

(c) Example of broadband RFI

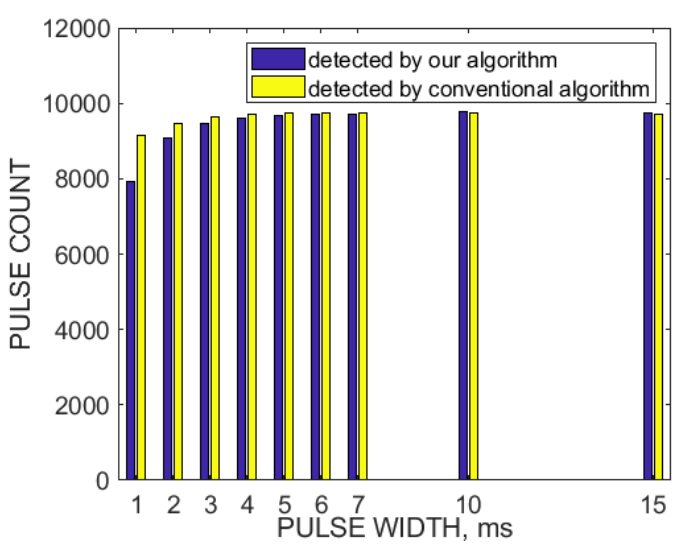

(b) Bar plot for the case of channelized RFI

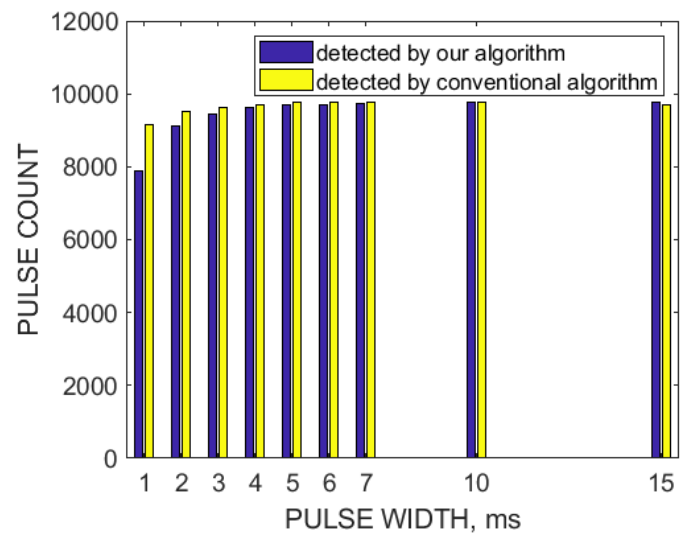

(d) Bar plot for the case of broadband RFI

Figure 4.11: Shown are two sets of bar plots displaying the dependence of the number of detected pulses by our and conventional algorithms on the width of pulses. In this experiment one fifth of the spectrogram is corrupted by RFI.

\section{Application of Convolutional Neural Network}

With increased computational capabilities in the past decades many applied sciences turned their attention towards the latest developments in computational sciences and particularly towards deep learning approaches that are shown to be successful in dealing with big data. Radio astronomy is not an exception. Deep learning approaches and methodologies are currently under the exploration by astronomers (see Sec. 1.3 for examples).

There are many practices and design patterns that can be used to construct an efficient architecture for a deep neural network. One of them is to explore the information in data when data are presented in different formats. Here we are investigating the ability of a 


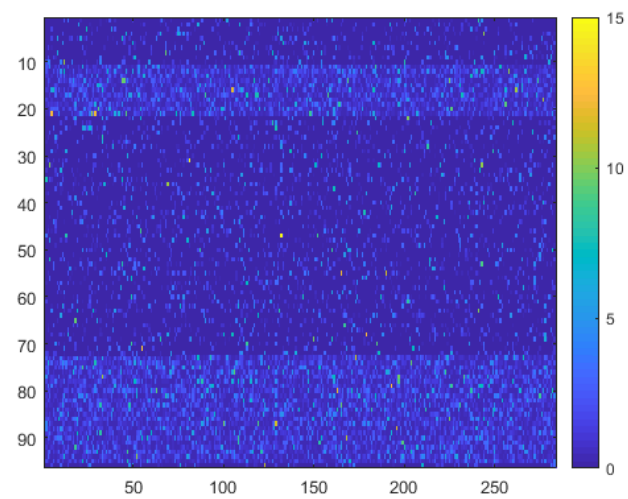

(a) Example of channelized RFI

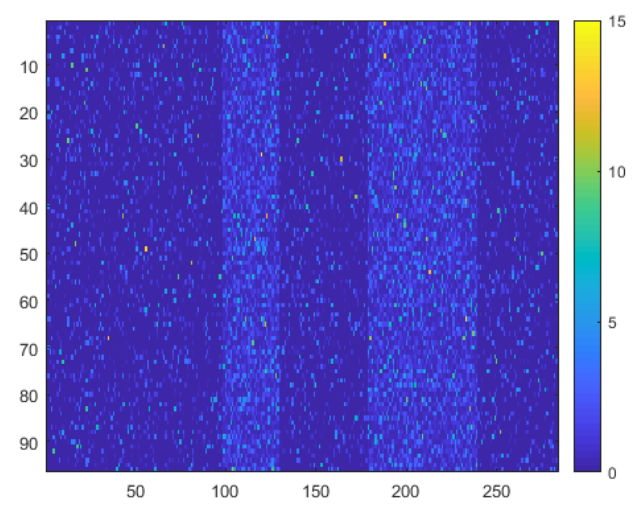

(c) Example of broadband RFI

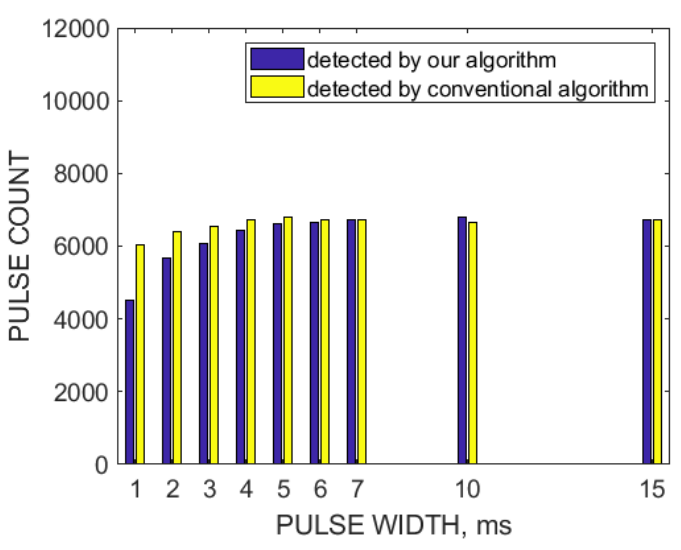

(b) Bar plot for the case of channelized RFI

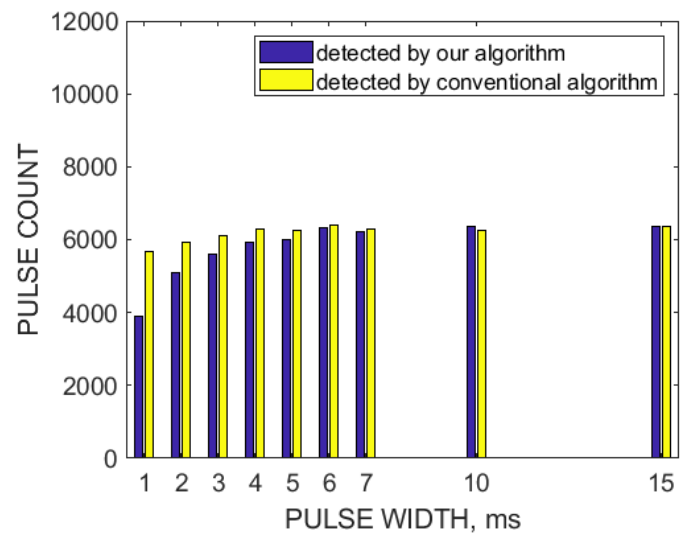

(d) Bar plot for the case of broadband RFI

Figure 4.12: Shown are two bar plots displaying the dependence of the number of detected pulses by our and conventional algorithms versus the width of pulses. In this example about one third of the spectrogram is corrupted by RFI.

deep neural network to extract informative features of an astronomical pulse at different processing stages as described by the block-diagram presented earlier in this chapter. A traditional approach in the development of deep-neural networks is to pick a pre-built and pre-tained network that has been used to solve a similar problem for another applied science field and tune to work with new type of data.

Transfer learning is a technique where pre-trained network is used for a new problem with few slight modifications. Transfer learning is suitable when there is not much data to train the network and computational resources are limited. In the analysis, we will be using a well-known off the shelf convolutional neural network called AlexNet [2]. AlexNet 


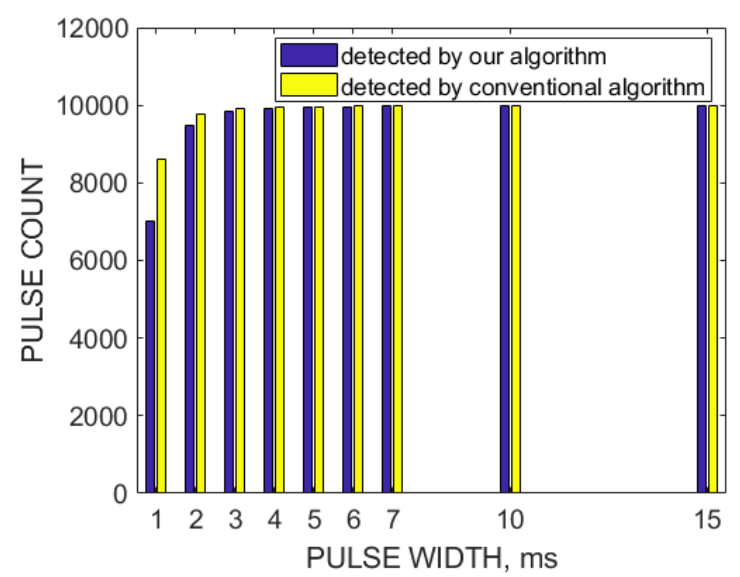

Figure 4.13: Shown is a bar plot displaying the dependence of the number of detected pulses by our and conventional algorithms versus the width of pulses. The number of simulated pulses for each DM is set to 1,000, and the value of DM is set to $300 \mathrm{pc} / \mathrm{cm}^{3}$.

is 8 layers deep and has 60 million parameters. It is designed to classify images in several different categories. It has been trained on a large image dataset (containing over a million of images). An illustration of the AlexNet's architecture is shown in Fig. 4.15.

We applied AlexNet to transformed spectrogram data as they appear on the output of different blocks in Fig. 4.1. We specifically applied AlexNet to the spectrogram data before linearization, spectrogram after linearization, and the data after the application of the Radon transform. For a fixed DM, fixed SNR, and fixed location of the pulse in a spectrogram the performance of AlexNet is evaluated and shown in Fig. 4.16. Here we use simulated data with the astronomical pulse dispersed at $\mathrm{DM}=300 \mathrm{pc} / \mathrm{cm}^{3}$ and the $\mathrm{SNR}$ value $\mathrm{SNR}=8$. Fig. 4.16(a) shows an example of the spectrogram used to train and test the third network Net 3. Fig. 4.16(b) shows an example of the spectrogram after linearization that is used to train and test the second network Net 2. Fig. 4.16(c) shows an example of the radon transformed data used to train and test the first network Net 1 . Net 0 refers to the algorithm proposed and presented in Sec. 4.2. For a higher SNR value (here we set it to $\mathrm{SNR}=10$ ), the results are shown in Fig. 4.17(a). In addition to exploring different formats of data we also analyzed the effect of varying some pulse parameters, for example DM and the location of the pulse. The results of the analysis are shown in Fig 4.17(b). These results were obtained after training the net with 10,000 training data. We can see clearly that adding 


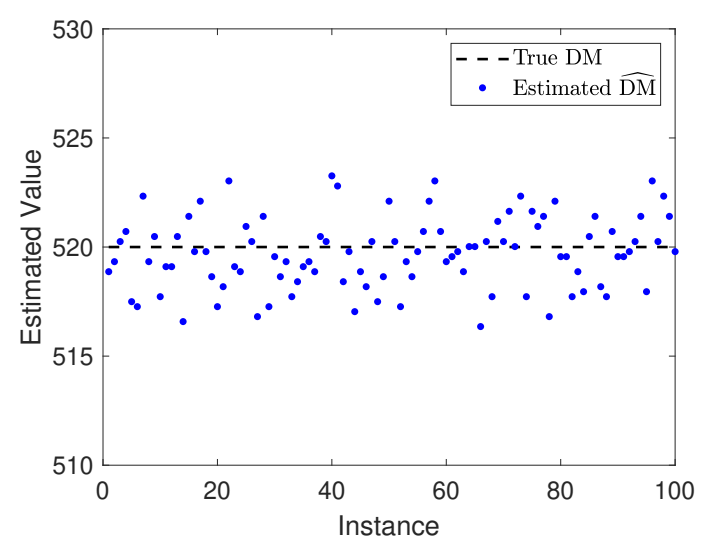

(a) FRB 180301

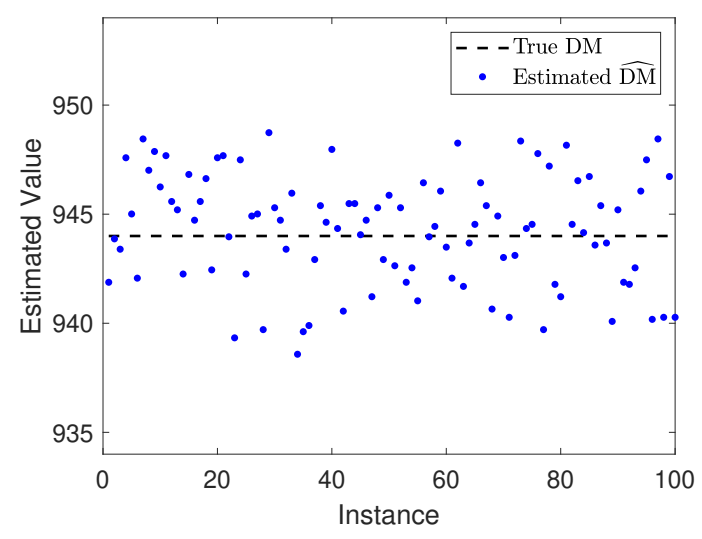

(c) FRB 110220

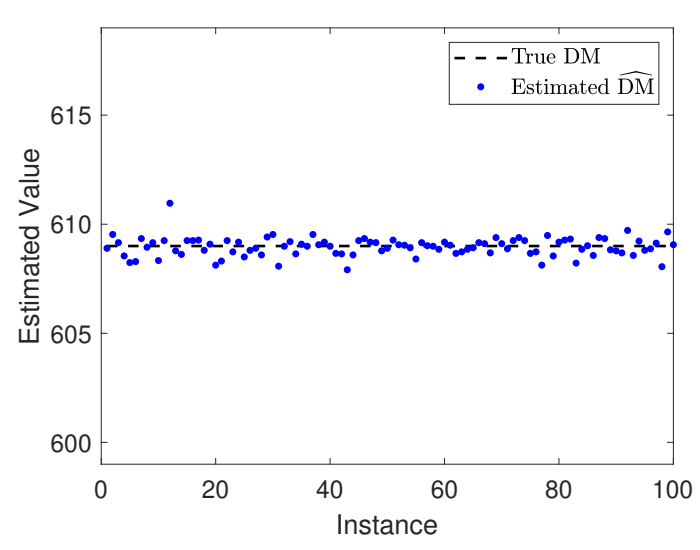

(b) FRB 170107

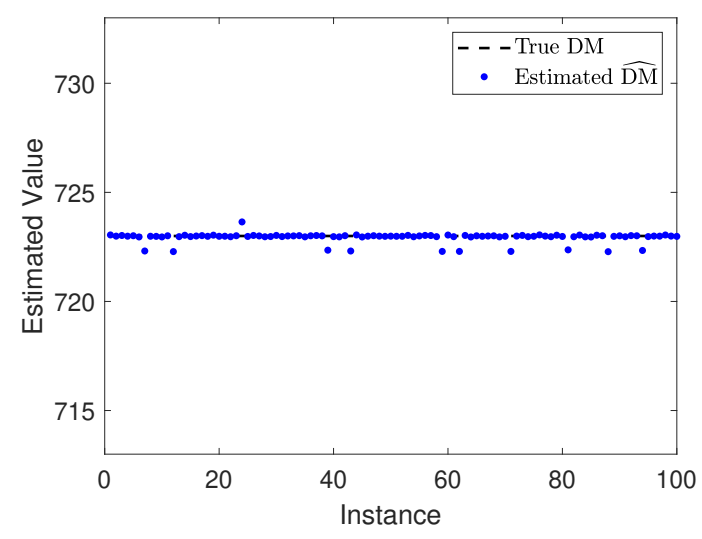

(d) FRB 110626

Figure 4.14: Shown are estimates of the DM value for several FRBs listed in Table 4.1. Each panel presents a plot of the estimated DM value versus the instance number and the true DM value.

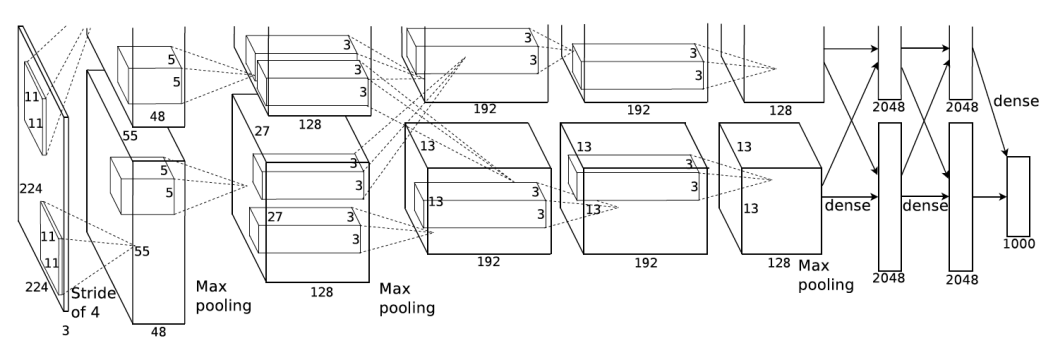

Figure 4.15: Illustration of the AlexNet architecture [2].

each step of our algorithm makes the net to be more capable of capturing the main features of the pulses. 


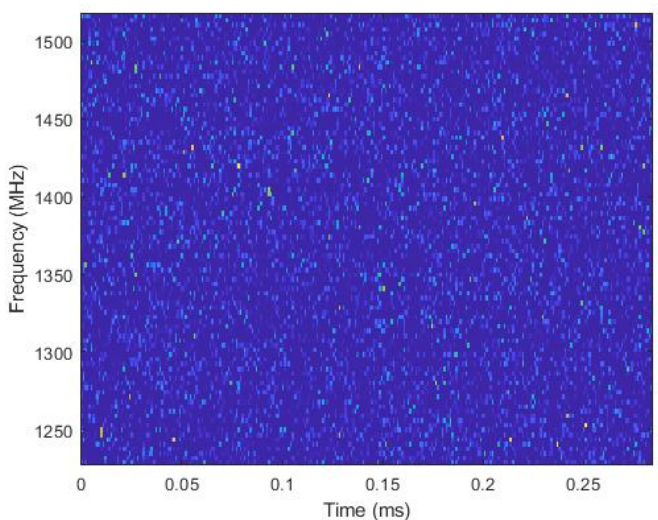

(a) Sample of input data for Net 3

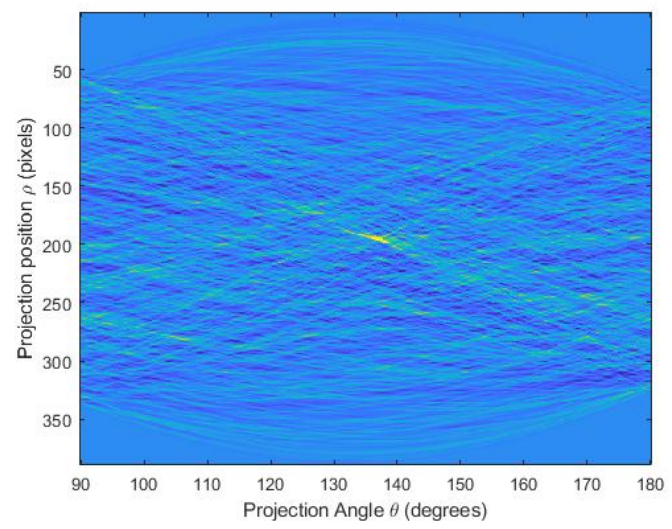

(c) Sample of input data for Net 1

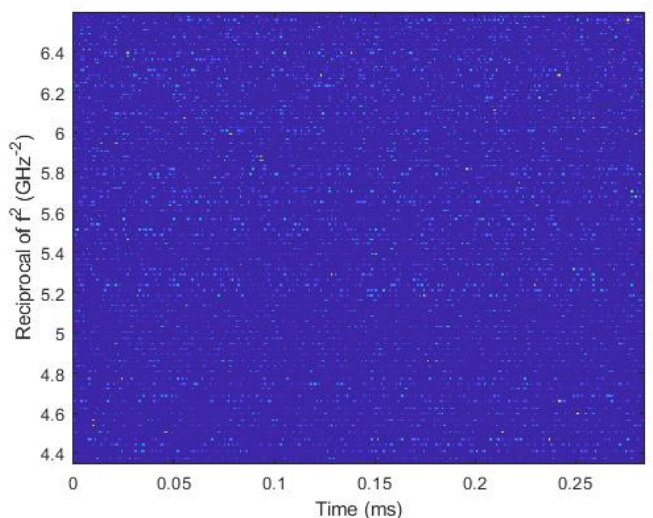

(b) Sample of input data for Net 2

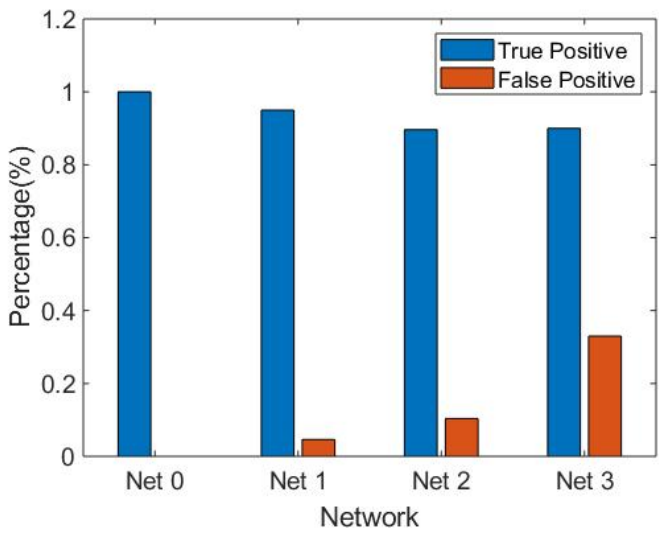

(d) The performance of AlexNet

Figure 4.16: Shown are the input data to AlexNet and the performance of AlexNet associated with the input data.

\subsubsection{Numerical evaluation based on experimental data}

In this section we intend to apply the detection and DM estimation approaches described in Sec. 4.2 and Sec. 4.3 to experimental data. We first analyze the performance of the proposed algorithms on the experimental data of Masui pulse, and then provide the performance analysis of the algorithms applied to experimental data of seven RRAT series.

\section{FRB 110523}

FRB 110523 was detected by Masui et al. [26] during their analysis of a data archive collected for the Green Bank Telescope neutral hydrogen 21-cm intensity mapping survey 


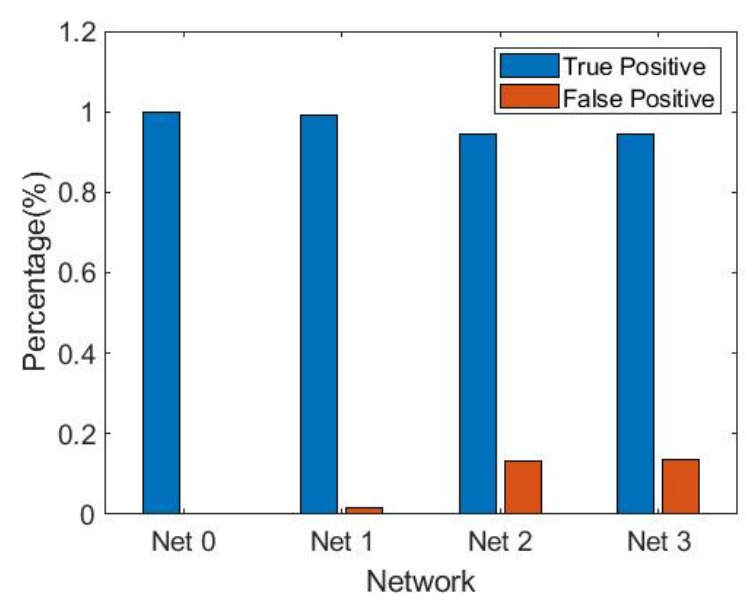

(a) $\mathrm{DM}=300 \mathrm{pc} / \mathrm{cm}^{3}$ and $\mathrm{SNR}=10$

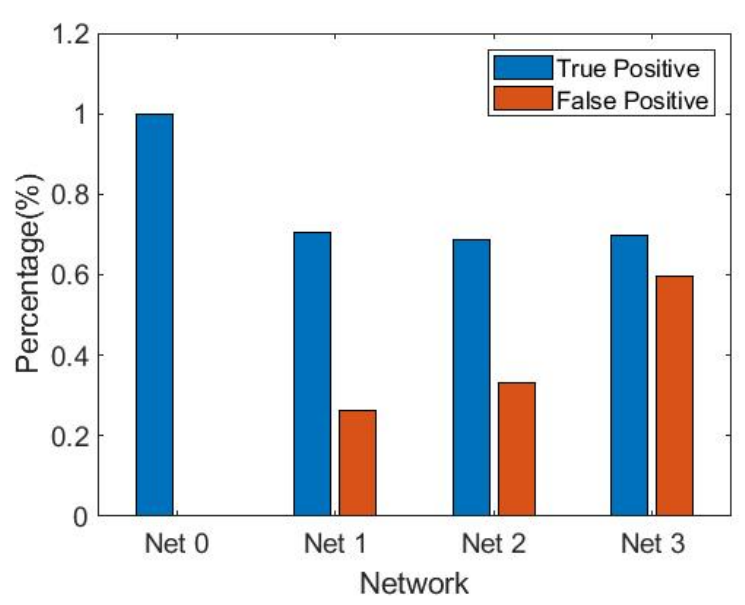

(b) $\mathrm{DM}$ is varied and $\mathrm{SNR}=10$

Figure 4.17: Shown are two bar plots summarizing the performance of AlexNet.

[39]. Prior to applying the proposed algorithm to data, we isolated the pulse from the rest of the provided data. The resulting spectrogram (enhanced for the pulse visibility) is shown in Fig. 4.18(a). In our experiments we apply our detection algorithm to raw data as they were provided to us.

The data are further transformed by linearizing them in frequency and then applying the Radon transform as shown in Fig. 4.18(b). After finding the maximum over the $\rho$-parameter of the Radon image for each given value of $\theta$, we plot the maximum value as a function of $\theta$ as shown in Fig. 4.18(d). Note that the signature of the Masui pulse appears as a sharp narrow pulse at the angle $\theta=170.40^{\circ}$.

From Eq. (4.12) we find that the estimated DM value of the Masui pulse is 623.18 $\mathrm{pc} / \mathrm{cm}^{3}$. Note that the estimate is reasonably close in its value to the true value of $\mathrm{DM}=$ $623.36 \mathrm{pc} / \mathrm{cm}^{3}$ and thus it can be used as an initial value for a more refined DM estimation due to the conventional algorithm.

\section{RRAT series}

In addition to the analysis above, our algorithms were tested on experimental data of seven RRATs collected at the Parkes Telescope. The temporal length of raw time series varied from 20 minutes to 60 minutes. The data were in the form of spectrograms each having 


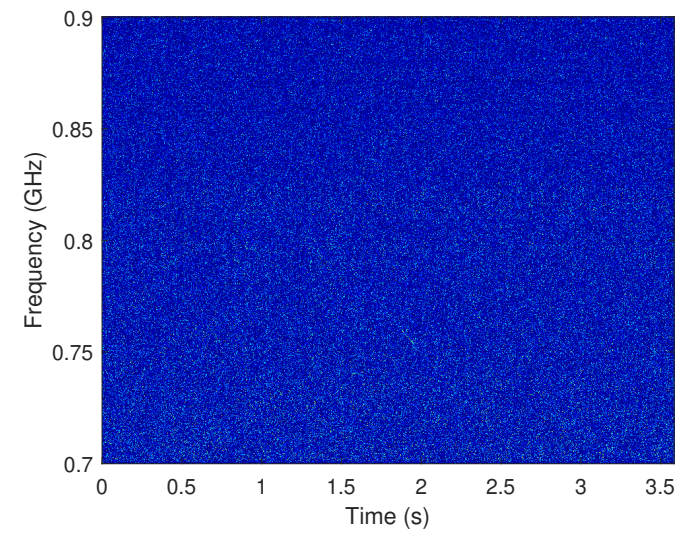

(a) Masui pulse enhanced for visualization

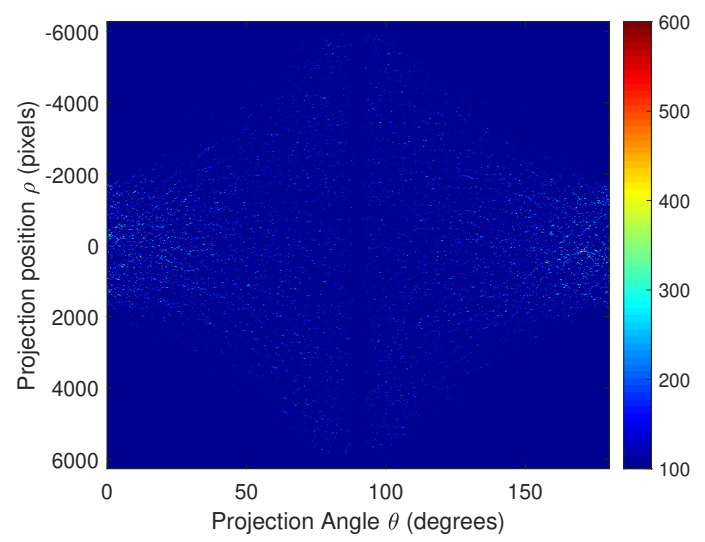

(c) Radon transform of the raw spectrogram

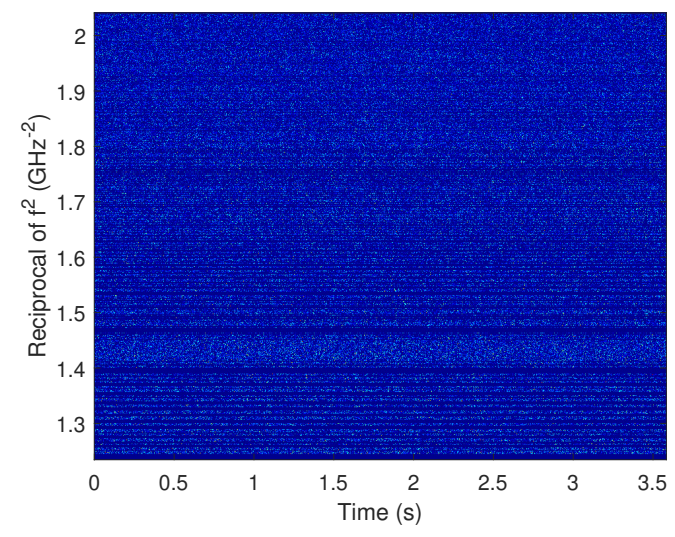

(b) Linearization of (a)

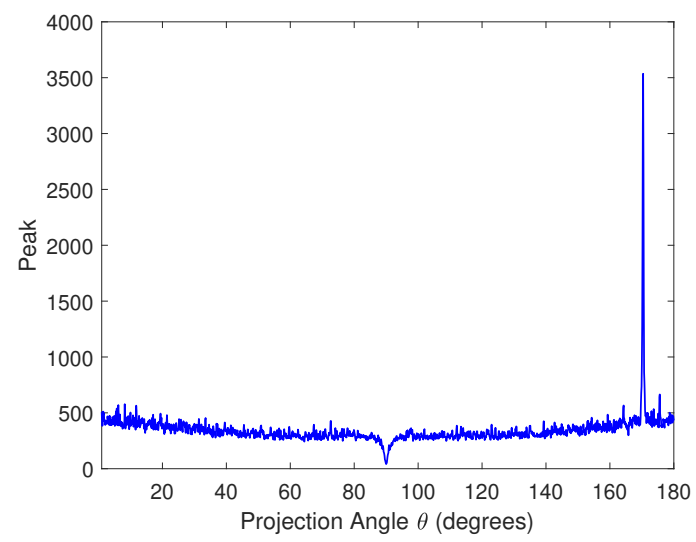

(d) Peak values in (c) as a function of $\theta$

Figure 4.18: Masui pulse spectrogram in (a) and its step-wise transformations in (b) through (d).

512 frequency channels and a temporal sampling interval $100 \mu s$. The Parkes Telescope was tuned to receive data in the range of frequencies between $1262 \mathrm{MHz}$ and $1518 \mathrm{MHz}$. The seven RRAT series used for performance analysis are listed in Table 4.2 together with their file names and several parameters describing each individual RRAT. These series have been previously analyzed by applying the conventional algorithm. The count of pulses detected by the conventional algorithm (and confirmed through manual inspection and diagnostic plots) is presented in the fifth column of Table 4.2. The pulses were detected at SNR $\geq 5$.

Prior to applying our algorithms to the RRAT series, they were preprocessed. Given the rotational period of a RRAT and the time of arrival of a strong pulse (both are determined by means of the conventinal algorithm), we used this information to partition each RRAT 
Table 4.2: A list of RRATs used in our numerical analysis.

\begin{tabular}{|c|c|c|c|c|c|c|}
\hline $\begin{array}{c}\text { Name of } \\
\text { RRAT }\end{array}$ & Data File & $\begin{array}{c}\text { DM } \\
\left(\mathrm{pc} / \mathrm{cm}^{3}\right)\end{array}$ & $\begin{array}{c}\text { Width } \\
(\mathrm{ms})\end{array}$ & $\begin{array}{c}\text { \# Detected } \\
\text { pulses }\end{array}$ & $\begin{array}{c}\text { \# New } \\
\text { candidates }\end{array}$ & $\begin{array}{c}\text { FA } \\
\text { Rate }\end{array}$ \\
\hline \hline J1819-1458 & RR0037_0151 & 196 & 3 & 48 & 1 & $2.57 \%$ \\
\hline J1317-5759 & RR0037_0071 & 145.4 & 10 & 13 & 1 & $0.03 \%$ \\
\hline J1913+1330 & RR0086_0181 & 175.64 & 2 & 7 & 0 & $0 \%$ \\
\hline J1839-0136 & RR0037_0031 & 293.4 & 15 & 1 & 0 & $0 \%$ \\
\hline J1826-1419 & RR0090_0121 & 159 & 2 & 16 & 1 & $5.26 \%$ \\
\hline J1047-58 & RR0057_0251 & 69.3 & 4 & 27 & 17 & $0.84 \%$ \\
\hline J1423-5647 & RR0070_0131 & 32.9 & 26.5 & 1 & 4 & $0.11 \%$ \\
\hline
\end{tabular}

spectrogram into windows such that each window contained either pure noise and RFI or a noisy pulse. Then our detection algorithm was applied to every window in the partitioned spectrogram.

Our algorithm confirmed every pulse detected by the conventional algorithm, and it also detected additional candidates which were missed by the conventional algorithm. The count of new candidates is listed in the sixth column of the table. Note that the table also reports the false alarm rate for every analyzed RRAT series. It is evaluated based on data after all windows with potential pulses were removed. Potential pulse locations were determined based on the time of arrival (TOA) of pulses reported by the conventional detection algorithm and also by taking into account the knowledge of the period of every RRAT.

Fig. 4.19(a) displays the estimates of DM values for the 49 pulses of RRAT J18191458 detected by the conventional and by our algorithms. The true DM value for this RRAT is $196 \mathrm{pc} / \mathrm{cm}^{3}$. Panels (b)-(d) in Fig. 4.19 demonstrate similar results for the files RR0037_0071, RR0057_0121, and RR0057_0251, respectively. The pulses marked in red are new candidates missed by the conventional algorithm, but detected by our algorithm. Note that the estimated DM values of the new candidates are reasonably close to the values of true DM of each RRAT. The true DM value is seen as a dashed line in each panel. 


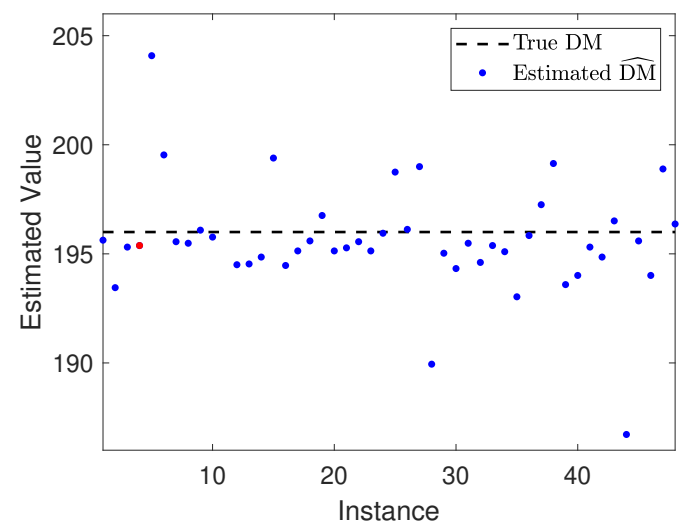

(a) File RR0037_0151 for RRAT J1819-1458

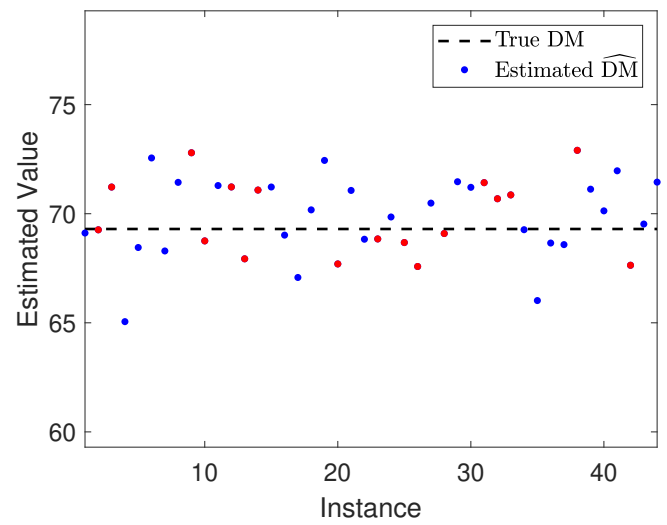

(c) File RR0057_0251 for RRAT J1047-58

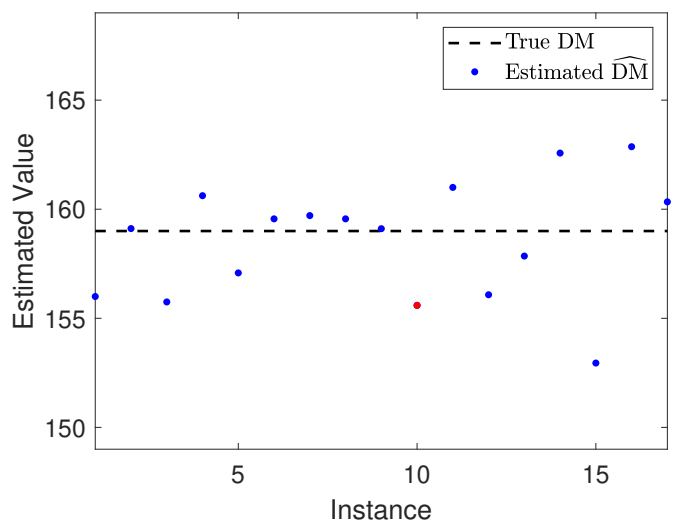

(b) File RR090_0121 for RRAT J1826-1419

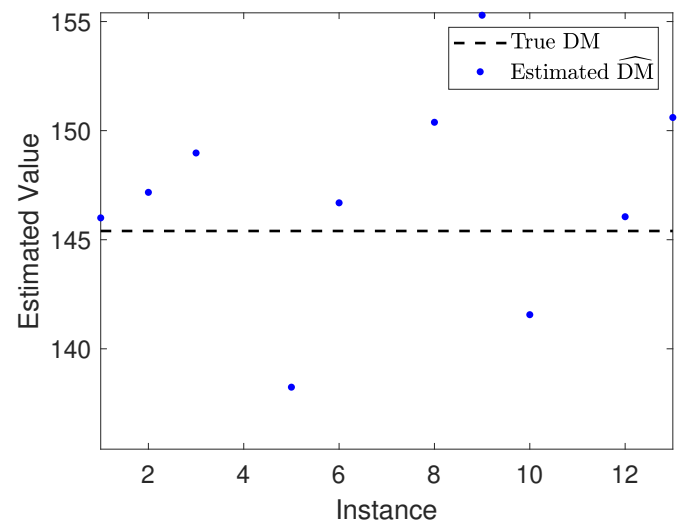

(d) File RR0037_0071 for RRAT J1317-5759

Figure 4.19: Estimated DM values for the detected pulses of four RRAT series. The new candidate pulses are marked in red.

\section{PSR B1937+21}

This part of the performance analysis uses a chunk of real data of the millisecond pulsar B1937+21. Fig. 4.20 shows the main steps of the periodic pulsar search algorithm proposed in this dissertation. The algorithm is applied to a spectrogram of the millisecond pulsar B1937+21. The chunk of PSR B1937+21 in Fig. 4.20(a) contains around 141 pulses that are not visible because of a low SNR value of each individual pulse. FFT of the projection at $\theta=138^{\circ}$ is shown in Fig. 4.20(e). The folded signal of the projection with a step equal to the reciprocal of the fundamental frequency of the pulsar in Fig. 4.20(e) results in Fig. 4.20(f), that is Rotation $(\mathrm{sec})=1 / 640 \mathrm{~Hz}$ which matches the true reported value of $1.55 \mathrm{~ms}$. The DM 
estimates of the pulsar obtained using Eq. 4.12 for different chunks of B1937+21 data are shown in Fig. 4.21.

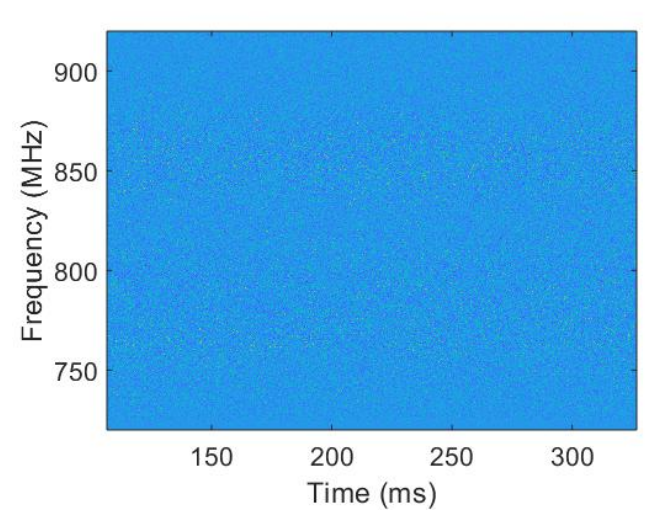

(a) Chunk of PSR B1937+21

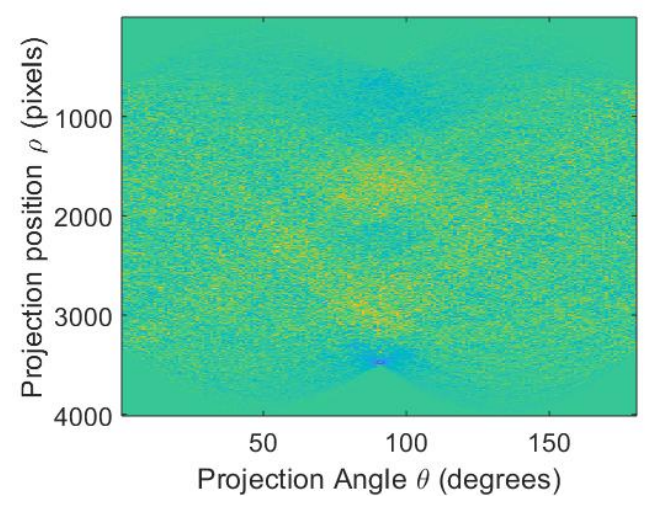

(c) Radon transform of (b)

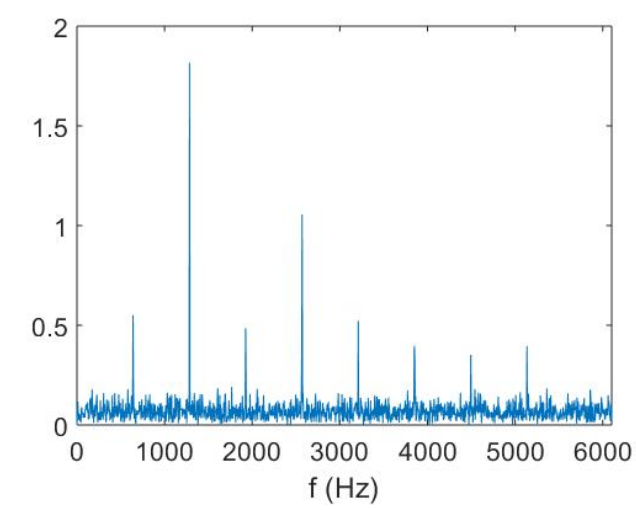

(e) FFT of (d)

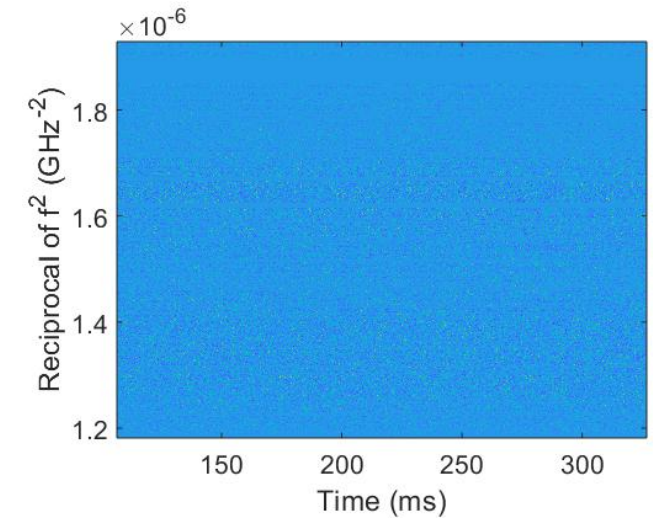

(b) Linearization of (a)

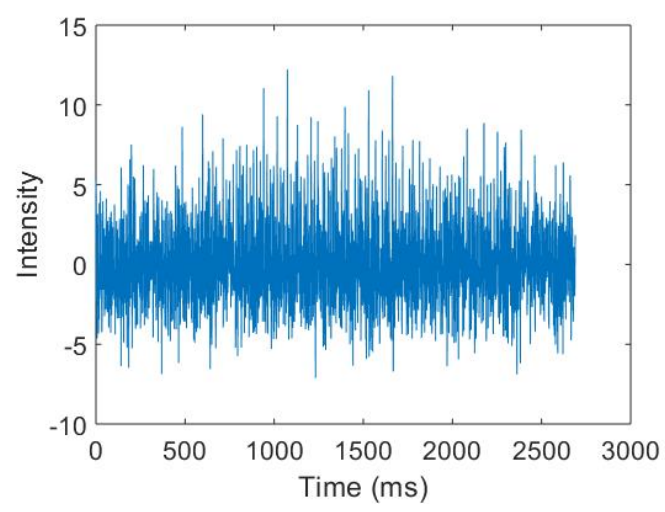

(d) Projection at $\theta=138^{\circ}$

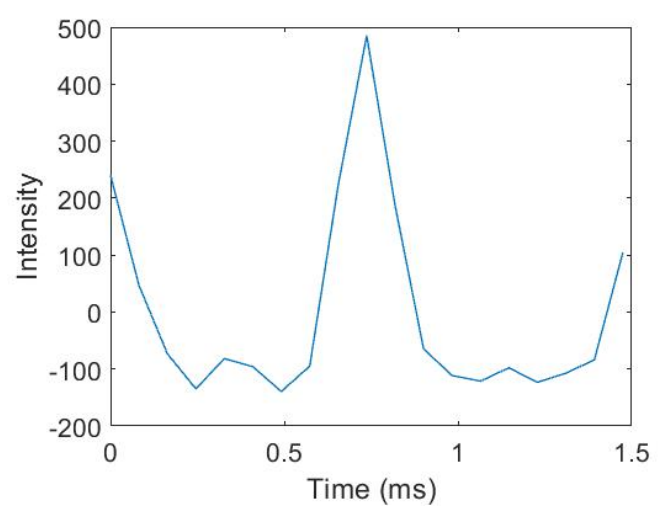

(f) Folding the signal (d)

Figure 4.20: Main steps of the detection approach in application to a spectrogram of the millisecond pulsar B1937+21 real data. 


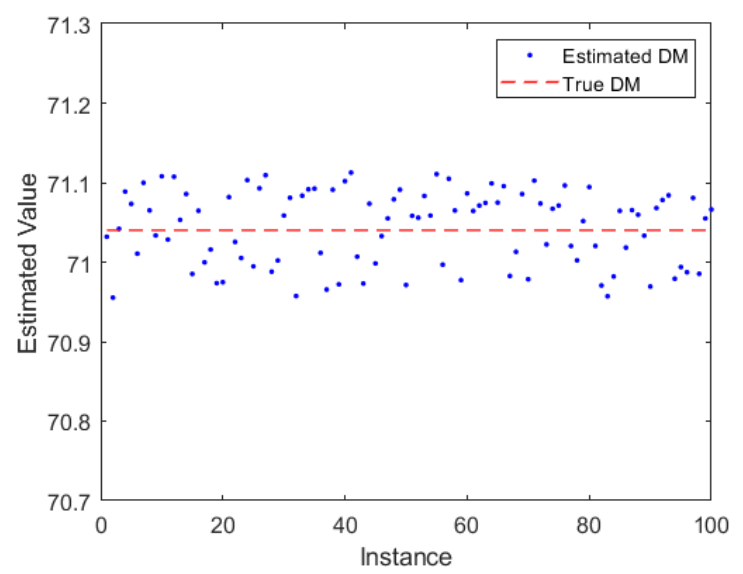

Figure 4.21: Estimated DM values for different chunks of B1937+21.

\subsection{Summary}

This chapter introduced a new algorithm for the detection of isolated astronomical pulses. The algorithm operates on spectrograms. The algorithm involves two transformations, frequency linearization and the Radon Transform, and comprizes of four main steps. In step one, we linearize the frequency dimension of a spectrogram. In step two, the Radon transform is applied to the linearized spectrogram. In step three, we apply smoothing to the output of the Radon Transform. In step four, we find the maximum of the smoothed Radon image along each column and display it as a function of angle $\theta$, then compare the maximum value of the function to the right of $90^{\circ}$ to the maximum standard deviation of the noise to the left of $90^{\circ}$.

Both experimental and simulated data were used to illustrate the performance of the detection algorithm. The results of our simulations demonstrate that regardless of the pulse width and the DM value of an FRB, the astronomical pulses are detectable at the SNR value set to 8. Apart from applying our detection algorithm to simulated data, we tested it on experimental data of the Masui pulse and of seven RRATs. The Masui pulse was easily detected by the proposed algorithm. In application to the RRAT series, the proposed detection algorithm was able to identify all pulses previously reported in the literature and in addition was able to find several new candidate pulses.

Once an astronomical pulse is detected, we applied a basic estimation approach to find 
an approximate DM value of the pulse. The results of performance analysis demonstrate that the estimated DM values are within narrow margins of the true DM value and that the margins depend on the instrumentation and the characteristics of data. 


\section{Chapter 5}

\section{SFT for the Detection of Isolated Astronomical Pulses}

\section{$5.1 \quad$ Introduction}

In this chapter, we modify the algorithm presented in Chapter 4. To accelerate its implementation, we engage the Spatial Fourier transform (SFT).

\subsection{Detection Algorithm}

The block diagram of the algorithm comprises three consecutive signal processing steps. First, we linearize the spectrogram of a pulse. Then we apply the SFT. Finally, we apply a detection rule. A brief description of each step is provided below.

\subsubsection{Linearization}

This is the first step of the algorithm, its intention is to transform a dispersed pulse into a linear function of time. This step has been previously introduced in Chapter 4 , for more details about this step please refer to 4.2.1. An example of a single simulated pulse is shown in Fig. 5.1(a) and an example of a periodic astrophysical signal is shown in Fig. 5.1(b). The linearized spectrograms of the two signals are presented in Fig. 5.1(c) and Fig. 5.1(d), respectively. A dispersed signal present in a linearized spectrogram is described 
by a continuous linear function relating a time delay of a signal and an inverse quadratic function of the corresponding channel frequency, with the unknown DM value as a scale factor.

Treating the linearized spectrogram as an image $\gamma(t, v)$ and assuming that the profile of an astronomical pulse is a rectangular function, the dispersed astronomical pulse in the spectrogram $\gamma(t, v)$ can be expressed as

$$
\gamma(t, v)=\Pi\left(\frac{t}{T}, \frac{v}{W}\right) * * \delta\left(t-t_{\text {ref }}-4.15 \times 10^{3} \times \mathrm{DM} \times\left(v-f_{\text {ref }}^{-2}\right)\right),
$$

where $\Pi\left(\frac{t}{T}, \frac{f}{W}\right)$ is a two-dimensional rectangular pulse defined over an area $T \times W, t_{r e f}$ is the reference time related to $f_{r e f}$, the parameter $T$ is the pulse width, "**" stands for a two-dimensional convolution, and $\delta(\cdot)$ is the Dirac delta function. In radio astronomy $f_{r e f}$ is often selected as the central frequency of the receiver bandwidth. In case of periodic pulses the astronomical pulses in the spectrogram are described mathematically as

$$
\gamma(t, v)=\Pi\left(\frac{t}{T}, \frac{v}{W}\right) * * \sum_{k=-\infty}^{\infty} \delta\left(t-t_{\text {ref }}-k T_{0}-4.15 \times 10^{3} \times \mathrm{DM} \times\left(v-f_{\text {ref }}^{-2}\right)\right)
$$

where $T_{0}$ is the rotational period of the periodic astrophysical signal.

\subsubsection{Spatial Fourier transform (SFT)}

The second step of the proposed approach is to apply the SFT to the linearized spectrogram $\gamma(t, v)$. Denote by $\Gamma(u, v)$ the SFT of the spectrogram. From 5.2 and the convolution property of the Fourier Transform, $\Gamma(u, v)$ is given as

$$
\Gamma(u, v)=T W \operatorname{sinc}(T u) \operatorname{sinc}(W v) A(u, v)
$$

where

$$
\begin{aligned}
A(u, v) & =\int_{t_{1}}^{t_{2}} \int_{r_{l}}^{r_{h}} \delta\left(t-t_{r e f}-4.15 \times 10^{3} \times \mathrm{DM} \times\left(r-f_{r e f}^{-2}\right)\right) \exp \{-j 2 \pi(t u+r v)\} d t d f \\
& =\exp \left(-j 2 \pi u\left(t_{r e f}+4.15 \times 10^{3} \times \mathrm{DM} f_{r e f}^{-2}\right)-j 2 \pi\left(v-4.15 \times 10^{3} \times \mathrm{DM} u\right)\left(\frac{r_{l}+r_{h}}{2}\right)\right)
\end{aligned}
$$

and $r=1 / f^{2}$. For the case of a periodic astronomical pulse, the STF is given by the following equation:

$$
\Gamma(u, v)=\frac{T W}{T_{0}} \sum_{k=-\infty}^{\infty} \operatorname{sinc}\left(\frac{T k}{T_{0}}\right) \operatorname{sinc}(W v) A\left(\frac{k}{T_{0}}, v\right) .
$$

where $u$ has be replaced with $k / T_{0}$. 


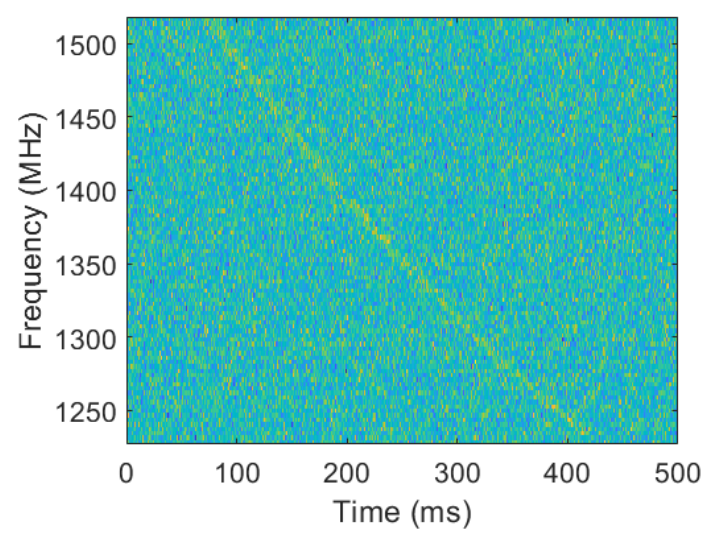

(a) Single pulse

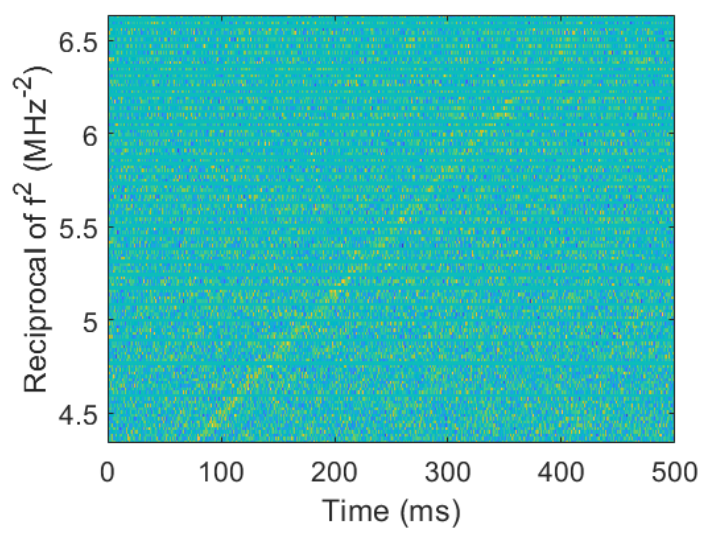

(c) Linearization of (a)

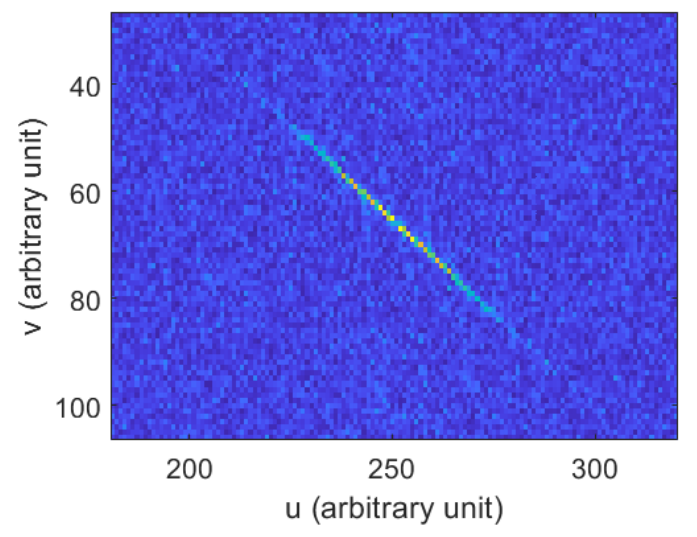

(e) SFT of (c)

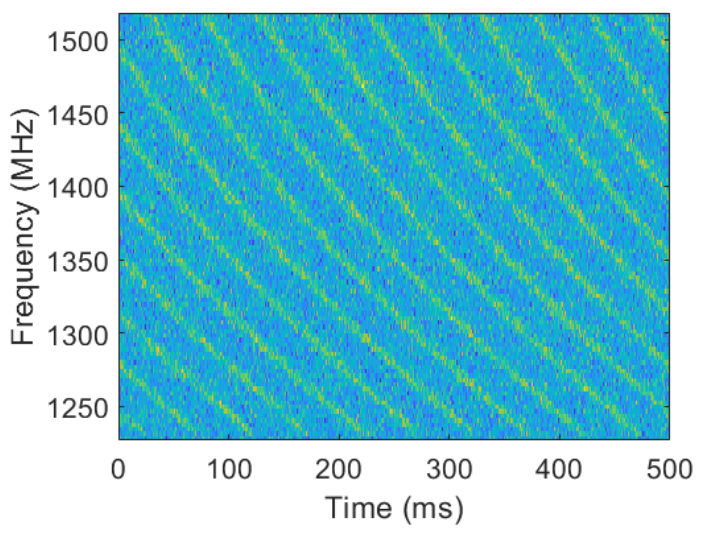

(b) Periodic pulse

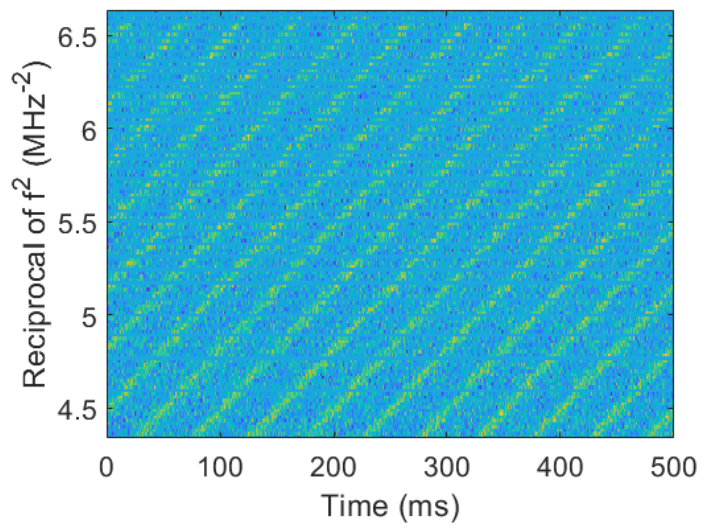

(d) Linearization of (b)

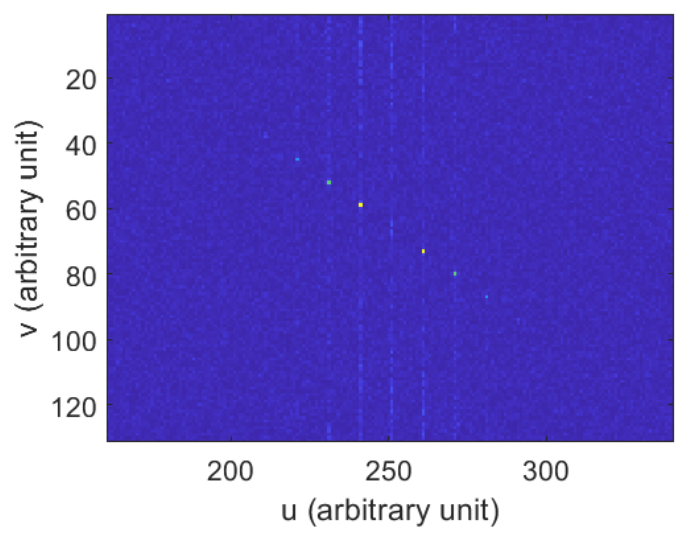

(f) SFT of (d)

Figure 5.1: Shown are an isolated pulse and a periodic signal and their step wise transformations. 


\subsubsection{Decision rule}

An astrophysical pulse can be detected by analyzing the magnitude of the Fourier transformed data. Denote the magnitude of the SFT as $X(u, v)$ and define the point $(N / 2, \tilde{K} / 2)$ as its center. The magnitude of $X(u, v)$ is displayed in Fig. 5.1(e) for the case when the data contains isolated pulses and in Fig. 5.1(f) for the case when the data contains periodic pulses. In Fig. 5.1(e), the signature of the pulse appears as a linear function with a nonzero slope, which is directly related to the slope of the original line that crosses the second and fourth quadrants. The signal duration (pulse width) of the original line in $(\mathrm{t}, \mathrm{f})$ space defines the length of the transformed line in the SFT space. The orientation of the transformed line is a function of the DM value. By applying this simple algebraic observation, we can develop many different detection rules. Here we will use the following rule:

$$
\sum_{u=N / 2}^{N} \sum_{v=\tilde{K} / 2}^{\tilde{K}} R(u, v) X(u, v)>\gamma \sum_{u=N / 2-N}^{N / 2} \sum_{v=\tilde{K} / 2-\tilde{K}}^{\tilde{K} / 2} R(u, v) X(u, v)
$$

where $R(u, v)$ is a two-dimensional filter response and $\gamma$ is a decision threshold $(\gamma>1)$. A pulse is detected if the above inequality holds and not detected, otherwise.

In Fig. 5.1(f), the signature of the periodic pulses appears as a set of relatively strong spikes (sharp peaks) in the second and fourth quadrants. Let $\sigma$ be the estimated standard deviation of the data in the first or third quadrants

$$
\sigma=\underset{\forall u<N / 2 \& \forall v>\tilde{K} / 2}{\operatorname{Std}} X(u, v) .
$$

These observations lead to the following decision rule:

$$
\max _{\forall u<N / 2 \& \forall v<\tilde{K} / 2} X(u, v)>\gamma \sigma,
$$

RFI is characterized by a zero DM value and may be broadband at a single time (bursty) or persistent at a single frequency. Such interference shows up along vertical and horizontal lines in SFT domain, and so may be nulled. Fig. 5.2 demonstrates an example of spectrograms corrupted with RFI and their signatures in the SFT domain. 


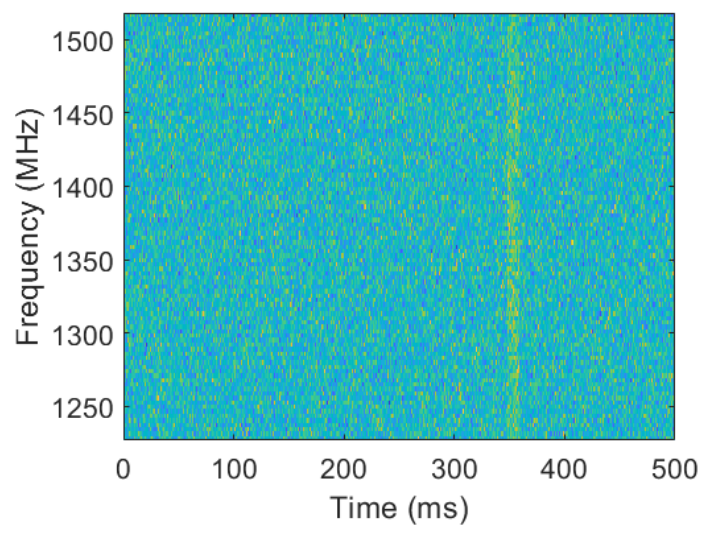

(a) Broadband, short-duration RFI

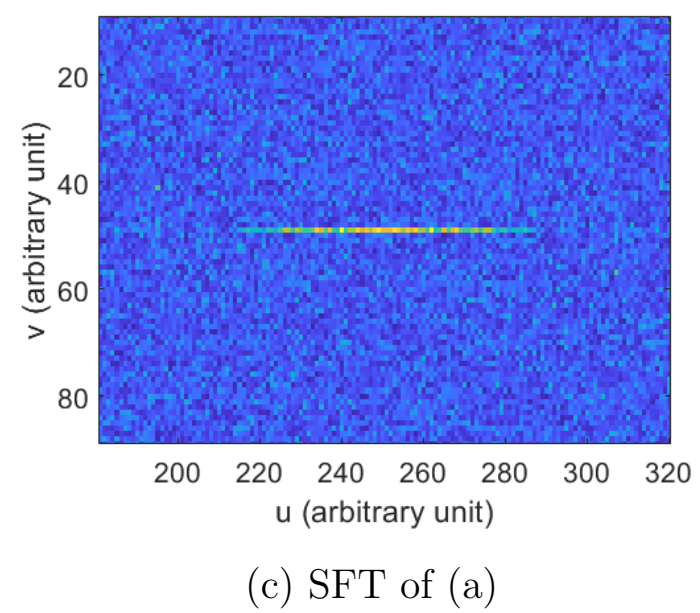

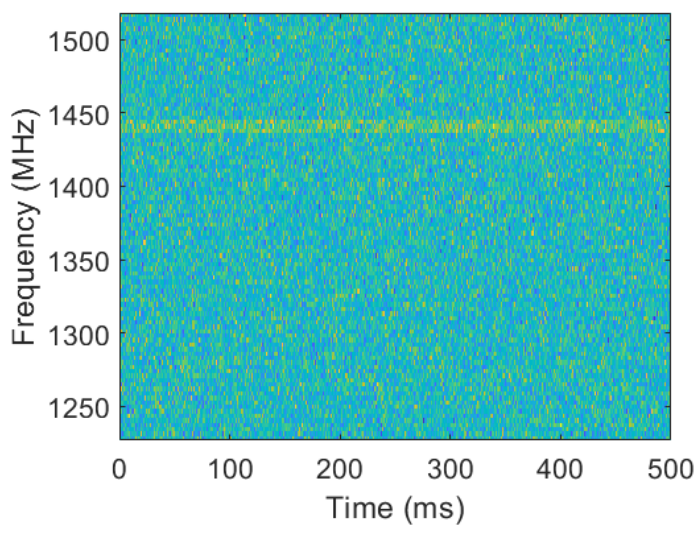

(b) Channelized RFI

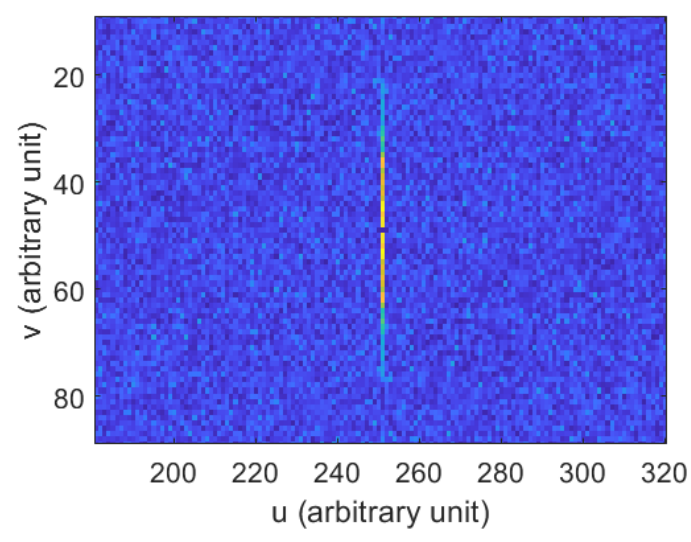

(d) SFT of (b)

Figure 5.2: Examples of RFI signals in spectrograms and in the SFT domain.

\subsubsection{Estimation of Parameters}

After a candidate pulse is detected, we can estimate the main pulse's parameters by locating the pulse signature in the SFT domain. We can develop a set of basic equations that relate the location of the signature in the SFT domain to the DM and also to the rotation in case of periodic pulse.

For the case of an isolated pulse, the dispersion equation describing the arrival of low frequencies of the pulse compared to the high frequencies of the pulse is given as

$$
t_{l}-t_{h}=\mathrm{DM} \times 4.15 \times 10^{3} \times\left(f_{l}^{-2}-f_{h}^{-2}\right)
$$

where $t_{l}$ and $t_{h}$ are the time instances of the pulse arrival to the lowest $f_{l}$ and the highest $f_{h}$ 
frequencies of the receiver band. The equation of the same line in the linearized spectrogram is

$$
t_{l}-t_{h}=\mathrm{DM} \times 4.15 \times 10^{3} \times\left(r_{h}-r_{l}\right)
$$

where $m_{S p c}$ is the slope of the line. From Eq. 5.8 and Eq. 5.9. DM estimate is given as:

$$
\widehat{\mathrm{DM}}=\frac{1}{m_{S p c} \times 4.15 \times 10^{3}}
$$

Given SFT data of a pulsar, we can relate the slope of the signature line of a linearized pulse in the magnitude SFT plot to the slope of the line in the linearized spectrogram

$$
m_{S p c}=m_{S F T} \times \frac{\left(r_{h}-r_{l}\right)}{N T_{s}},
$$

where $T_{s}$ is the the sampling interval and $\tilde{K}$ is the number of discrete frequency channels in the linearized spectrogram. After substituting Eq. 5.11 into Eq. 5.10, the formula for the DM estimate can be rewritten in terms of $m_{S F T}$ as

$$
\widehat{\mathrm{DM}}\left(N, T_{s}\right)=\frac{N T_{s}}{m_{S F T} \times 4.15 \times 10^{3} \times\left(f_{l}^{-2}-f_{h}^{-2}\right)} .
$$

The slope of the line that connects the center point $(N / 2 ; \tilde{K} / 2)$ to the location of the peak value of the SFT domain can be used as $m_{S F T}$ and the Eq. 5.13 can be used to estimate the DM for the periodic pulses. Furthermore, the location of the peak value in the $u$ dimension of the SFT domian can be used to estimate the rotation of a pulsar. The rotation can be found as follows

$$
R=\frac{2 N T_{S}}{\left(u_{0}-N / 2\right)}
$$

where $u_{0}$ is the location of the peak value in the u-axis and $N$ is the number of bins along u-axis.

\subsection{Performance Analysis}

In this section the performance of the detection and DM estimation algorithms is evaluated. The performance of the detection algorithm is demonstrated through Detection Rate at zero empirical False Alarm Rate [38]. Due to a very limited amount of practical data containing real FRB pulses, our numerical evaluation involving FRBs mostly relies on simulated 
data. RRAT series used in this evaluation were provided by the Department of Physics and Astronomy at West Virginia University [12].

\subsubsection{Numerical Evaluation based on Simulated Data}

The initial data are displayed in filter bank format, where astrophysical pulses are dispersed according to Eq. 5.1. We assume that the pulse profile of each astrophysical pulse is Gaussian with the FWHM set to correspond to the width of pulse of interest. The bandwidth of the simulated filter bank data, sampling interval, the number of discrete frequency channels, the pulse SNR, and the DM value of a simulated pulse are set to the values provided in [5]. To add noise to a simulated astrophysical pulse, we apply the conventional definition of the SNR, where it is defined as a ratio of the peak value of a de-dispersed and matched-filtered observed signal, to the root mean square (RMS) of the simulated noise. A detailed description of our simulation approach is provided in [30].

\section{Pulse Detection}

To analyze the detection performance of the developed algorithm we simulate the FRBs listed in Table 5.1, we generate 100 realizations per FRB. For each FRB we also generate 100 spectrograms of pure noise. The decision rule (see Eq. 5.5) is applied to both noisy data containing a pulse and to the data containing noise only. The value of parameter $\gamma$ is selected such that the estimated False Alarm Rate (based on 100 realizations of pure noise) is zero. The last column in Table 5.1 presents the Detection Rate for each analyzed FRB. From this example, the developed algorithm shows promise in terms of detecting astrophysical pulses parameterized by a large DM value. Also note that the algorithm is able to detect both wide and narrow pulses as well as pulses down to SNR of 11.5.

\section{The effect of pulse width on the detection performance}

The results of the previous subsection demonstrate that all simulated pulses detectable by the conventional algorithm are also detectable by the developed algorithm. In this subsection we numerically analyze the effect of the pulse width on the detection performance. 
Table 5.1: A list of fast radio bursts used in our numerical analysis.

\begin{tabular}{|c|c|c|c|c|c|}
\hline Name & Telescope & $\begin{array}{c}\text { DM } \\
\left(\mathrm{pc} / \mathrm{cm}^{3}\right)\end{array}$ & $\begin{array}{c}\text { Width } \\
(\mathrm{ms})\end{array}$ & SNR & $\begin{array}{c}\text { Detection } \\
\text { Rate }\end{array}$ \\
\hline \hline FRB121102 & Arecibo & 557 & 3 & 14 & $100 \%$ \\
\hline FRB110220 & Parkes & 944.38 & 5.6 & 49 & $100 \%$ \\
\hline FRB160608 & UTMOST & 682 & 9 & 12 & $100 \%$ \\
\hline FRB170107 & ASKAP & 609 & 2.6 & 16 & $100 \%$ \\
\hline FRB180301 & Parkes & 520 & 3 & 16 & $100 \%$ \\
\hline FRB180311 & Parkes & 1575.6 & 12 & 11.5 & $100 \%$ \\
\hline
\end{tabular}

To demonstrate this, we simulated 1,000 astronomical pulses for two values of DM, 300 $\mathrm{pc} / \mathrm{cm}^{3}$ and $1500 \mathrm{pc} / \mathrm{cm}^{3}$, and several values of the pulse width. The input SNR in all simulations was set to 8. Fig. 5.3 shows the count of detected pulses, that is the count of the pulses with estimated SNR above 5, as a function of the pulse width for the DM value of $300 \mathrm{pc} / \mathrm{cm}^{3}$ in the left panel and for the DM value of $1500 \mathrm{pc} / \mathrm{cm}^{3}$ in the right panel, respectively.

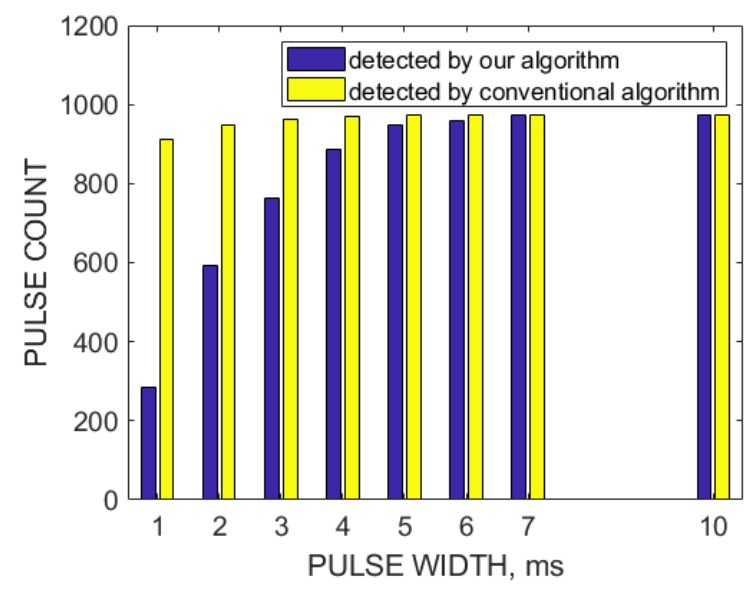

(a) $\mathrm{DM}=300 \mathrm{pc} / \mathrm{cm}^{3}$

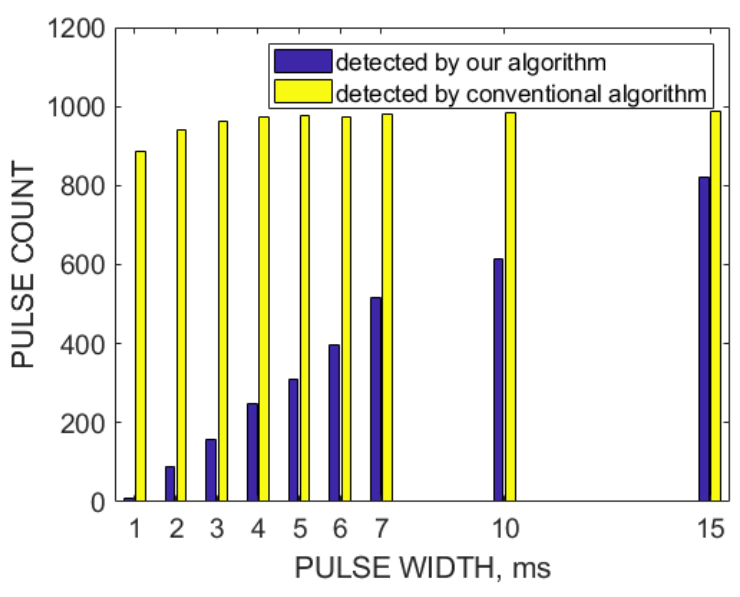

(b) $\mathrm{DM}=1500 \mathrm{pc} / \mathrm{cm}^{3}$

Figure 5.3: Shown are two bar plots displaying the dependence of the number of detected pulses by our and conventional algorithms versus the width of pulses. The input SNR is set to 8 , the number of simulated pulses for each DM is set to 1,000 , and the value of DM is set to $300 \mathrm{pc} / \mathrm{cm}^{3}$ in the left panel and to $1500 \mathrm{pc} / \mathrm{cm}^{3}$ in the right panel. 


\section{DM Estimation}

We also apply the DM estimation approach outlined in Sec. 5.2.4 to the simulated data described above. Fig. 5.6 displays two plots of the estimated DM values for each generated instance. The estimated values are shown as blue dots. The dashed horizontal line represents the true DM value of each FRB, the values of the true DM are listed in Petroff et al. [5]. A close inspection of each plot in Fig. 5.6 shows that the estimated DM value for each simulated FRB lies within relatively narrow margins of the true value of DM. The margins are completely dependent on the instrumentation and the characteristics of the data.

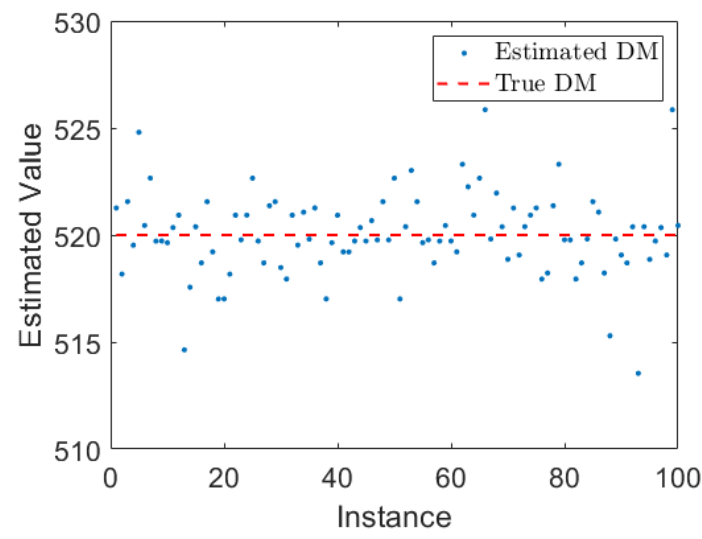

(a) FRB 180301

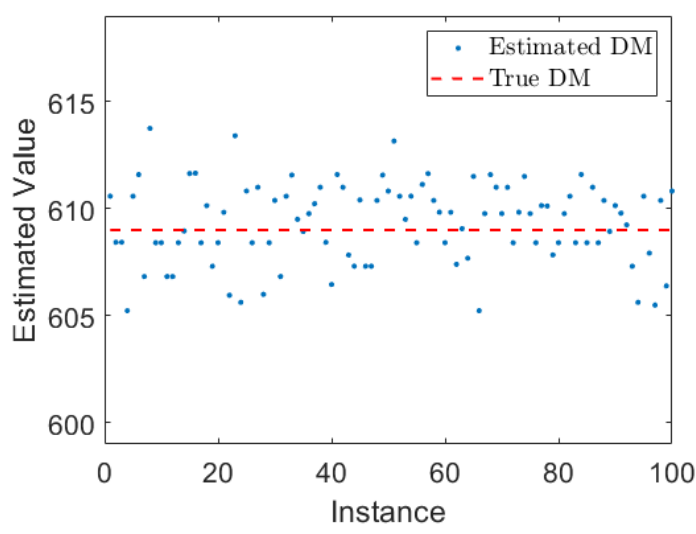

(b) FRB 170107

Figure 5.4: Shown are estimates of the DM value for two FRBs listed in Table 5.1. Each panel presents a plot of the estimated DM value versus the instance number and the true DM value.

\subsubsection{Numerical evaluation based on experimental data}

In this section we intend to apply the detection and DM estimation approaches described in Sec. 5.2.3 and Sec. 5.2.4 to experimental data. We first analyze the performance of the proposed algorithms on the experimental data of Masui pulse, and then provide the performance analysis of the algorithms applied to experimental data of seven RRAT series. 


\section{FRB 110523}

FRB 110523 was detected by Masui et al. [26] during their analysis of a data archive collected for the Green Bank Telescope neutral hydrogen 21-cm intensity mapping survey [39]. Prior to applying the proposed algorithm to data, we isolated the pulse from the rest of the provided data. The resulting spectrogram (enhanced for the pulse visibility) is shown in Fig. 5.5(b). In our experiments we apply our detection algorithm to raw data as they were provided to us.

The data are further transformed by linearizing them in frequency and then applying the SFT as shown in Fig. 5.5(d). Note that the signature of the Masui pulse appears as a line crossing the second and fourth quadrants around the origin of the coordinate system. The slope of the line is $\tan \left(59.8^{\circ}\right)$.

From Eq. (5.13) we find that the estimated DM value of the Masui pulse is 623.4252 $\mathrm{pc} / \mathrm{cm}^{3}$. Note that the estimate is reasonably close in its value to the true value of $\mathrm{DM}=$ $623.36 \mathrm{pc} / \mathrm{cm}^{3}$ and thus it can be used as an initial value for a more refined DM estimation due to the conventional algorithm.

For Lorimer burst, the slope of the signature line in the SFT as in Fig. 5.5(c) is $\tan \left(65.22^{0}\right)$ and the estimated DM value is $374.201 \mathrm{pc} / \mathrm{cm}^{3}$. Note that the estimate is reasonably close in its value to the true value of $\mathrm{DM}=375 \mathrm{pc} / \mathrm{cm}^{3}$.

\section{RRAT series}

In addition to the analysis above, our algorithms were tested on experimental data of three RRATs collected at the Parkes Telescope. The temporal length of raw time series varied from 20 minutes to 60 minutes. The data were in the form of spectrograms each having 512 frequency channels and a temporal sampling interval $100 \mu s$. The Parkes Telescope was tuned to receive data in the range of frequencies between $1262 \mathrm{MHz}$ and $1518 \mathrm{MHz}$. The three RRAT series used for performance analysis are listed in Table 5.2 together with their file names and several parameters describing each individual RRAT. These series have been previously analyzed by applying the conventional algorithm and the count of pulses detected was archived on Bowser. 


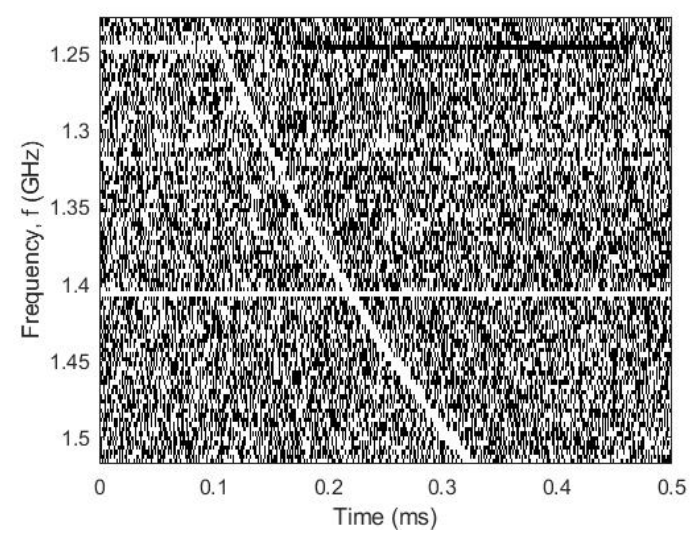

(a) Lorimer burst

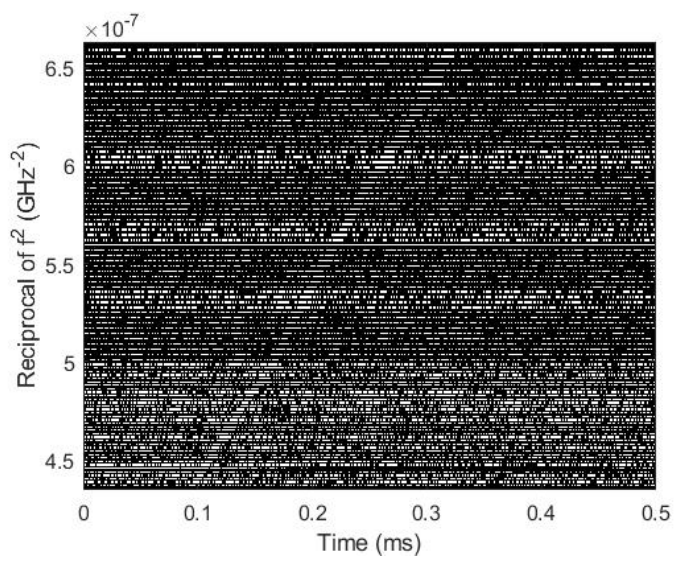

(c) Linearization of (a)

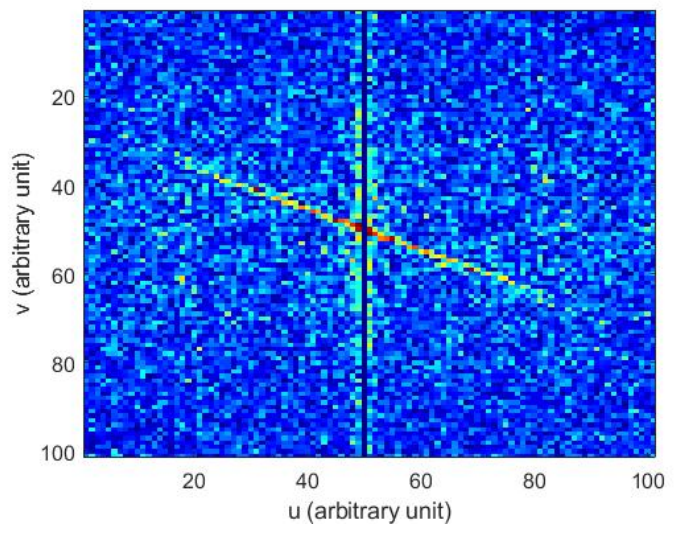

(e) SFT of (c)

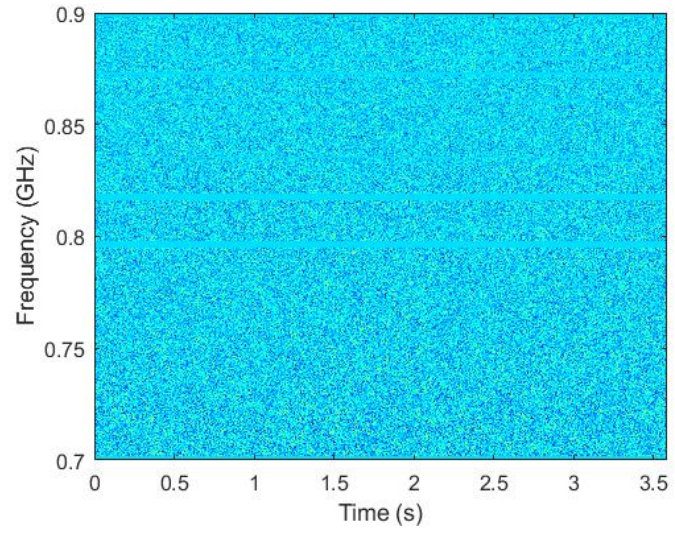

(b) Masui pulse

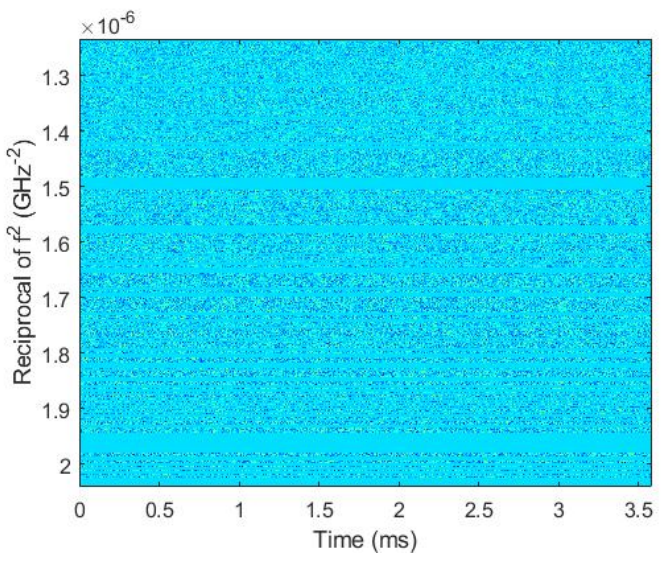

(d) Linearization of (b)

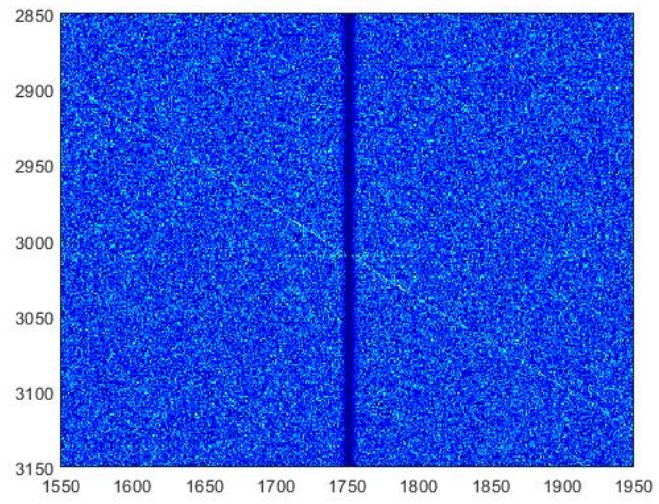

(f) SFT of (d)

Figure 5.5: Spectrograms of the experimental data of Lorimer burst (shown in panel(a)) and of Masui pulse (shown in panel (b)) and their step wise transformations. 
Table 5.2: A list of RRATs used in our numerical analysis.

\begin{tabular}{|c|c|c|c|c|c|}
\hline $\begin{array}{c}\text { Name of } \\
\text { RRAT }\end{array}$ & Data File & $\begin{array}{c}\text { DM } \\
\left(\mathrm{pc} / \mathrm{cm}^{3}\right)\end{array}$ & $\begin{array}{c}\text { Width } \\
(\mathrm{ms})\end{array}$ & $\begin{array}{c}\text { \# Detected } \\
\text { pulses }\end{array}$ & $\begin{array}{c}\text { Detection } \\
\text { Rate }\end{array}$ \\
\hline \hline J1819-1458 & RR0037_0151 & 196 & 3 & 48 & $100 \%$ \\
\hline J1317-5759 & RR0037_0071 & 145.4 & 10 & 7 & $100 \%$ \\
\hline J1047-58 & RR0057_0251 & 69.3 & 4 & 27 & $100 \%$ \\
\hline
\end{tabular}

Our algorithm confirmed every pulse detected by the conventional algorithm. Potential pulse locations were determined based on the time of arrival (TOA) of pulses reported by the conventional detection algorithm and also by taking into account the knowledge of the period of every RRAT.

Fig. 5.6(a) displays the estimates of DM values for the 48 pulses of RRAT J1819-1458 detected by the conventional and by our algorithms. The true DM value for this RRAT is 196 pc/ $\mathrm{cm}^{3}$. Fig. 5.6 demonstrates similar results for the file RR0037_0071. Note that the estimated DM values are reasonably close to the values of true DM of each RRAT. The true DM value is seen as a dashed line in each panel.

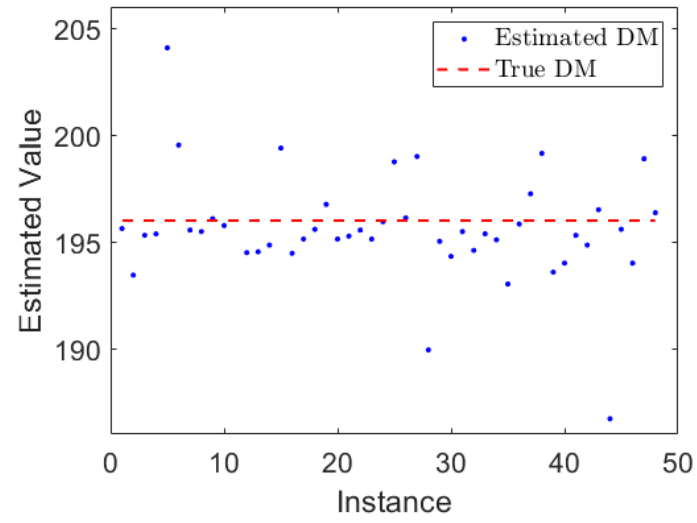

(a) File RR0037_0151 for RRAT J1819-1458

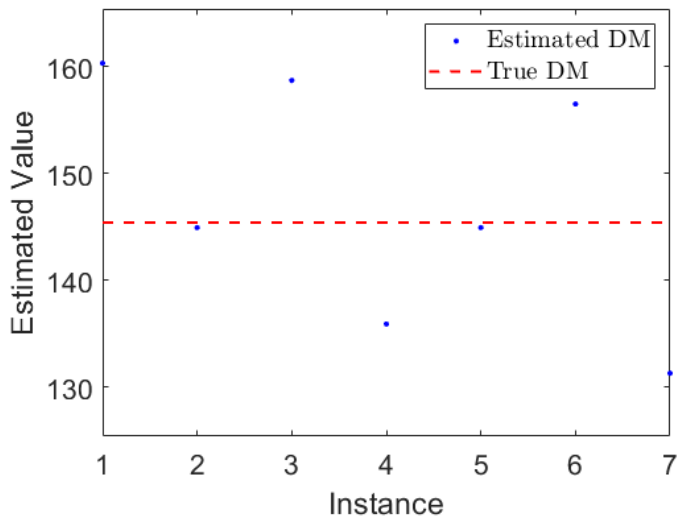

(b) File RR0037_0071 for RRAT J1317-5759

Figure 5.6: Estimated DM values for the detected pulses of two RRAT series. 


\section{PSR B1937+21}

The real data of the millisecond pulsar B1937+21 is used in this part of the analysis. Fig. 5.7 shows the main steps of the periodic search of our algorithm as being applied to a spectrogram chunk of the millisecond pulsar B1937+21. The chunk of PSR B1937+21 in Fig. 5.7(a) contains around 141 pulses that are not visible because of the a SNR value of the individual pulses. After applying the SFT to the data, we can see a few pulses of the pulsar by eye. After finding the maximum value along each column of Fig. 5.7(c) we obtain the plot shown in Fig. 5.7(d). The rotation of the pulsar can be estimated from finding the locations of the peaks. As demonstrated in Fig. 5.7 (d), the tallest peak is observed at $u_{0}=810$, where $u$ is measured in bins (dimensionless units). Let $N$ be the number of bins along $\mathrm{u}$-axis and $T_{s}$ be the sampling interval (for example, $T_{s}=81.92 \mu s$ for the Green Bank Telescope [19]), then the location of the peak value is related to the rotational period $R$ as follows $u_{0}=N / 2+2 T_{s} N / R$.

For PSR B1937+21 shown in Fig. 5.7, $u_{0}=810$ pixels and $N=2050$, then

$$
R=\frac{2 N T_{S}}{\left(N / 2-u_{0}\right)}=\frac{2 \times 2050 \times 81.92 \times 10^{-3}}{2050 / 2-810}=1.5622 \mathrm{~ms}
$$

which is matching the true reported value [41].

The DM value can be estimated by locating the peak in the SFT space which is located at $\left(v_{0}=1207, u_{0}=810\right)$ in Fig. 5.7 (d), the $\left(v_{0}=1207, u_{0}=810\right)$ which indicated that the slope is $m_{S F T}=0.7611$. From Eq. (5.13) we find that the estimated DM value is 71.1154 $\mathrm{pc} / \mathrm{cm}^{3}$. Note that the estimate is reasonably close in its value to the true value of $\mathrm{DM}=$ $71.0249 \mathrm{pc} / \mathrm{cm}^{3}$

\subsection{Summary}

In this chapter, we expanded the algorithm presented in Chapter 3 . The modified algorithm involves two additional steps, frequency linearization and the application of the SFT to the linearized spectrogram. Both practical and simulated data were used to illustrate the performance of the developed approaches. The complexity of the detection algorithm is dominated by the complexity of the SFT, hence the approach can be used to detect new 


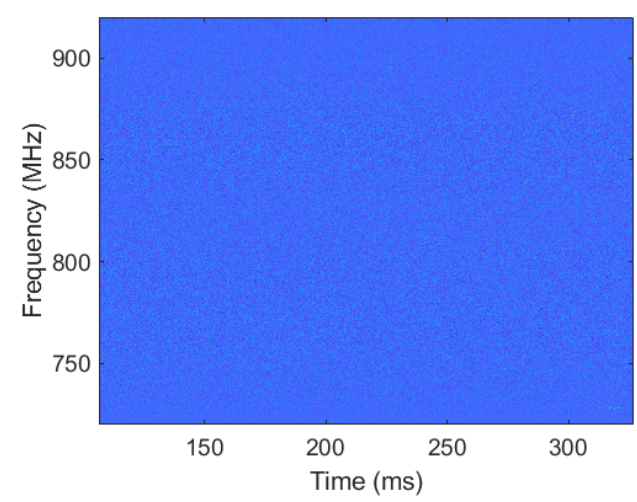

(a) Chunk of PSR B1937+21

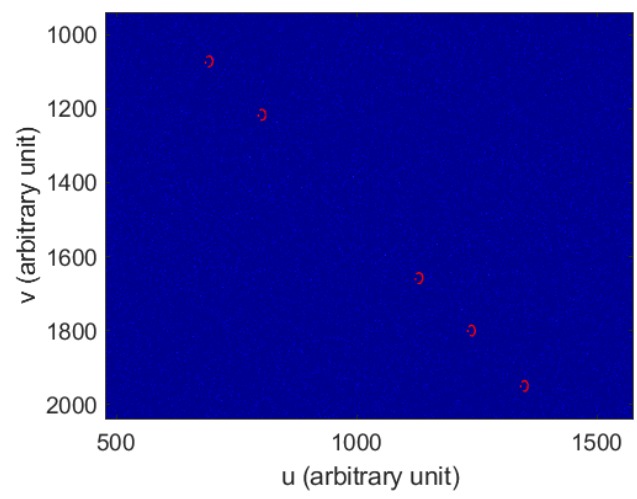

(c) $\operatorname{SFT}(b)$

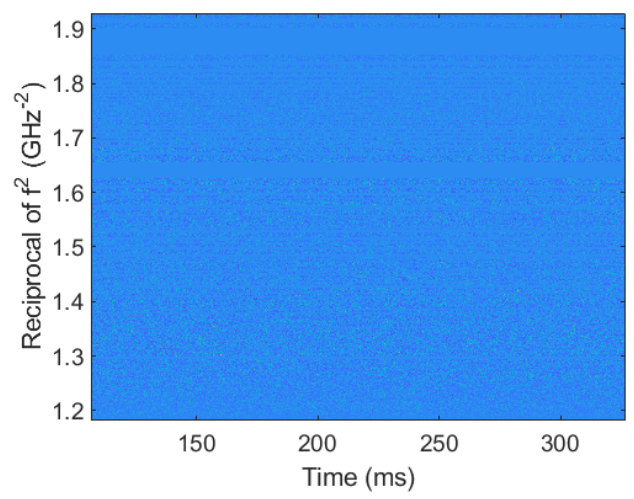

(b) Linearization of (a)

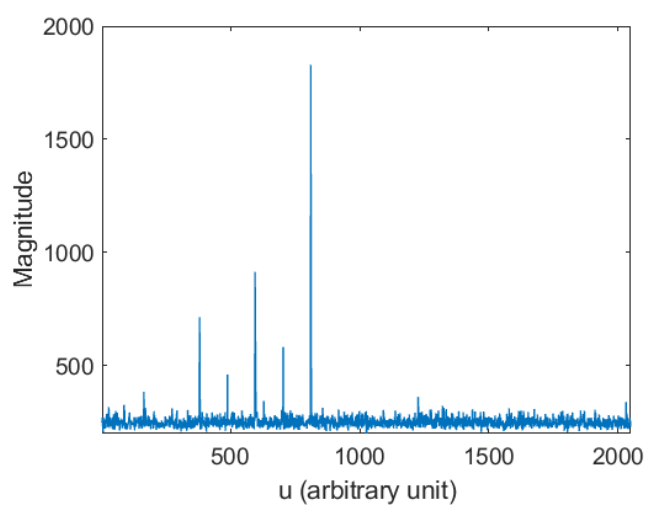

(d) Maximum of (c) over $v$ on the 1st and 2nd quadrants

Figure 5.7: Main steps of the detection approach in application to a spectrogram chunk of the millisecond pulsar B1937+21.

pulsars in real time. The complexity of the SFT can be further reduced by applying FFT to the dimension of the spectrogram with lowest number of bins and then apply FFT along the other dimension for a narrow range of low frequencies. The results of our simulations demonstrate that the algorithm is sensitive to pulse width and DM value, the astronomical pulses are detectable at the SNR value set to 8 when pulse width is more than 5 bins and DM value at $300 \mathrm{pc} / \mathrm{cm}^{3}$. Apart from applying our detection algorithm to simulated data, we tested it on experimental data of the Masui pulse, Lorimer burst, and three RRATs. The Masui pulse and Lorimer burst were easily detected by the proposed algorithm. In application to the RRAT series, the proposed detection algorithm was able to identify all pulses previously reported in the literature.

Once an astronomical pulse is detected, we applied a basic estimation approach to find 
an approximate DM value of the pulse. The results of performance analysis demonstrate that the estimated DM values are within narrow margins of the true DM value and that the margins depend on the instrumentation and the characteristics of data. 


\section{Chapter 6}

\section{Detection in Radio Astronomy Dense Aperture Arrays}

\subsection{Introduction}

Dense Aperture Arrays (DAA) have been used for wide view radar imaging and in satellite transmitters for quite a long time [42,43]. Their wide field-of-view (FOV) makes them a valuable tool in effective searches of targets in military applications [44]. Recently DAAs have also found their application in radio astronomy, where an array of antenna elements is installed on a radio telescope instead of a traditional horn receiver. While a horn receiver is capable of imaging only a very small fraction of the sky at a specific time instance, a telescope equipped with a DAA can monitor a much larger area of the sky, giving an order of magnitude or more increase in survey speed, at a comparable spatial and temporal resolution. The design of DAAs for radio astronomy applications has faced a large number of challenges, with the primary challenge that of operating at extremely low Signal-to-Noise Ratios (SNR). After many years of careful research and developments of DAAs for radio astronomy applications, several prototype DAAs have been delivered [45]. Many of them are undergoing extensive testing and some have already been installed on antennas for initial test and commissioning [46]. As an example, a collaboration between Brigham Young University and the National Radio Astronomy Observatory has developed a 19-element DAA using room temperature dipoles and cooled low noise amplifiers. The array has been optimized 
with respect to spillover noise, illumination pattern and the beam-forming operation [47,48]. The electronics backend including a digital signal processing unit has been designed. After its final testing this summer, the array feed will be commissioned on the Green Bank Telescope (GBT) next year.

One of the most important applications of astronomical array feeds is to search for new millisecond pulsars and other short duration radio transients. Discoveries made in the past several years have shown that the radio sky is very dynamic. Many known sources have shown new behavior, and new classes of sources have been discovered. Among them are the Rotating Radio Transients (RRATs) [49] and Fast Radio Bursts (FRBs) 3, 4]. They have been attributed to a variety of celestial phenomena, from coalescing neutron stars to magnetic reconnection of a neutron star with its exploded companion. Traditional single pixel receivers have a very narrow field-of-view and so their survey speed for transients is slow. The introduction of low noise array feeds makes discovery of new fast transients much more likely.

The array feed designed for the GBT outputs a number of measurements such as the integrated energy of beamformed data and pairwise integrated correlation functions between the outputs of the array elements. While this ensures that processing and storing of raw data can be performed in real time, this does not guarantee the optimal use of the information in the raw array measurements.

The main goal of this work is to offer a complimentary (or possibly alternative) approach to extracting informative representations and statistics from raw data that can be shaped into decision rules for reliable detection of FRBs and other transient signals. We are looking at this problem from the perspective of classical detection and estimation theory 50, 51. We propose two models underlying the raw data received by the elements of an array feed.

The remainder of this chapter is organized as follows. Sec. 6.2 describes the assumptions and stochastic models used in this work. Sec. 6.3 is devoted to the derivation of the two detection rules. Sec. 6.4 demonstrates the feasibility of using the developed rules for automated detection of FRBs. Sec. 6.5 concludes the chapter with a brief summary and some suggestions for future developments. 


\subsection{Assumptions and Models}

Modern radio telescopes are built to receive astronomical signals over a broad frequency range partitioned into multiple narrow frequency channels. While traveling through the celestial environment filled with free electrons, an astronomical signal experiences dispersion. The multichannel design of telescope receivers ensures that the signal can be de-dispersed and integrated over the channels. This in turn boosts the SNR of the signal and allows detection of radio frequency interference.

A basic incoherent de-dispersion algorithm is based on evaluating the time delay between the arrival of the astronomical signal in successive frequency channels. The time delay $\Delta t$ is proportional to $D M /\left(f_{\text {chan }}^{2}-f_{\text {ref }}^{2}\right)$, where $f_{\text {chan }}$ and $f_{\text {ref }}$ are the central frequencies of a channel and a reference frequency, $f_{\text {chan }}>f_{\text {ref }}$, and $D M$ is dispersion measure evaluated as an integral of the density of electrons in the space over the distance from the astronomical source of interest to the telescope on the ground. The original de-dispersion algorithm is computationally expensive, mostly because the distance that the signal of interest has traveled is unknown to us, which results in a very large number of trials to find a good alignment of signals in two frequency channels [52]. In this work we assume that the raw data have been de-dispersed using a traditional de-dispersion approach prior to any other signal processing of the data. We leave the development of a new de-dispersion algorithm that will be specifically applied to array data for a work in the future.

Radio telescopes are optimized with respect to the receiver noise. Array feeds are supplied with cryogenic coolers to ensure low electronic noise. In spite of all efforts to lower the level of noise, the SNRs for the detection of radio astronomy sources are very low (the order of -30 to $-50 \mathrm{~dB})$. Researchers identify a number of different noise sources in arrays of feeds. These include spillover noise; coupling noise between elements of an array feed; sky noise; thermal noise from array antenna elements and amplifier noise. The combined noise is modeled as complex Gaussian noise with a cumulative correlation function that can be estimated through a calibration procedure.

In radio astronomy, source signals (FRBs and other transients) are often modeled as spatio-temporal stochastic processes. Their spatio-temporal flux densities are measured in 
Janskys. Most pulsars have extremely steep radio spectra. Typical pulsar spectral indices are $\alpha \sim 1.7\left(\mathrm{~S} \propto \nu^{-1.7}\right)$, although some may be steeper or flatter (see the handbook by Lorimer and Kramer [52]).

\subsection{Inferential Statistics}

Given the assumptions and models introduced in the previous section, our goal is to develop statistical decision rules for the automated detection of FRBs and other transients in spatially correlated noise. With the storage space and storage data rates being the main constraints, the focus of the work is on extracting compact informative statistics included in decision rules.

Classical detection and estimation theory offers two distinct approaches to model parameters and signals of interest. They may be treated as deterministic or random. Under these two assumptions the probabilistic optimization process yields different algorithms, which can be compared to determine which performs best with the given sensor data.

\subsubsection{Detection Using a Deterministic Source Signal Model}

We will first consider using a deterministic model for the astronomical signal of interest. The typical raw output of an array feed is a vector of temporally sampled voltage functions. The raw voltages may contain an astronomical signal emersed in an additive noise. Denote by $s[n], n=1, \ldots, N$ the temporal samples of the signal integrated over $10-100 \mu$ sec intervals. Although, it is not very intuitive, we view the integrated signal as a deterministic function of time, observed in additive Gaussian noise. We consider a DAA with $J$ elements and assume that DAA element signals are spatially correlated, element to element, but temporally independent, and that the noise correlation function, denoted as $\mathbf{R}_{\epsilon}$ is unknown. The $J$-length array response vector a is assumed to be known or well estimated during DAA calibration (see $47,48,53,54)$ ).

Consider $N$ temporal DAA array vector samples $\mathbf{x}[1], \ldots, \mathbf{x}[N]$. Two states of nature are being tested: (1) the data do not contain the signal of interest and thus are composed of samples of noise only and (2) the signal of interest is present and mixed with noise. 
Associated with the two states of nature are two hypotheses:

$$
\begin{array}{ll}
H_{0}: \mathbf{x}[n]=\epsilon[n] & \sim C N\left(0, \mathbf{R}_{\epsilon}\right), n=1, \ldots, N, \\
H_{1}: \mathbf{x}[n]=\mathbf{a} s[n]+\epsilon[n] & \sim C N\left(\mathbf{a} s[n], \mathbf{R}_{\epsilon}\right), n=1, \ldots, N,
\end{array}
$$

where $\sim C N(\cdot, \cdot)$ implies that $\mathbf{x}[n]$ is distributed as complex Gaussian.

We will also use $L$ observations of secondary data (noise only) denoted as $\mathbf{x}[N+1], \ldots, \mathbf{x}[N+$ $L] \sim C N\left(\mathbf{0}, \mathbf{R}_{\epsilon}\right)$ collected separately and known to be "source signal free."

We will assume that the detector is designed to implement the Generalized Likelihood Ratio Test (GLRT), which for this problem is stated as follows:

$$
\Lambda=\frac{\max _{\mathbf{s}, \mathbf{R}_{\epsilon}} p_{1}(\mathbf{x}[1], \ldots, \mathbf{x}[N+L])}{\max _{\mathbf{R}_{\epsilon}} p_{0}(\mathbf{x}[1], \ldots, \mathbf{x}[N+L])},
$$

where $p_{0}(\cdot)$ and $p_{1}(\cdot)$ are two conditional probability density functions of observed data under the two alternative hypotheses $H_{0}$ and $H_{1}$ respectively and $\max _{z}(f(z))$ denotes the maximization of an argument $f(z)$ with respect to the parameter $z$.

The noise covariance matrix $\mathbf{R}_{\epsilon}$ maximizing $(6.2)$ under $H_{0}$ and $H_{1}$ is given as

$$
\begin{gathered}
\hat{\mathbf{R}}_{0}=\frac{1}{N+L} \sum_{n=1}^{N+L} \mathbf{x}[n] \mathbf{x}[n]^{H} \\
\hat{\mathbf{R}}_{1}=\frac{1}{N+L}\left(\sum_{n=1}^{N}(\mathbf{x}[n]-\mathbf{a} \hat{s}[n])(\mathbf{x}[n]-\mathbf{a} \hat{s}[n])^{H}+\sum_{n=N+1}^{N+L} \mathbf{x}[n] \mathbf{x}[n]^{H}\right),
\end{gathered}
$$

where the superscript $H$ denotes "Hermitian transposed" and $\hat{\mathbf{R}}_{0}, \hat{\mathbf{R}}_{1}$ are the estimates parameterizing $H_{0}$ and $H_{1}$, respectively. (A careful derivation of the result is provided in the text by Muirhead [55].) Note that under $H_{1}$ the matrix $\mathbf{R}_{1}$ and the unknown signal vector s have to be estimated jointly.

Define $\mathbf{S}=\sum_{n=N+1}^{N+L} \mathbf{x}[n] \mathbf{x}[n]^{H}$.

Simultaneous estimation of $\mathbf{s}$ and $\mathbf{R}_{1}$ leads to the following maximizer of $\mathbf{s}$ :

$$
\hat{s}[n]=\frac{\mathbf{a}^{H} \mathbf{S}^{-1} \mathbf{x}[n]}{\mathbf{a}^{H} \mathbf{S}^{-1} \mathbf{a}}, n=1, \ldots, N .
$$

After substituting 6.3), 6.4 , and 6.5 into 6.2,

$$
\Lambda=\frac{\left(\operatorname{det} \hat{\mathbf{R}}_{1}\right)^{-(N+L)}}{\left(\operatorname{det} \hat{\mathbf{R}}_{0}\right)^{-(N+L)}}
$$


The test statistic $\Lambda$ can be replaced with

$$
l=\frac{\operatorname{det}\left(\hat{\mathbf{R}}_{0}\right)}{\operatorname{det}\left(\hat{\mathbf{R}}_{1}\right)},
$$

rewriting 6.3) and (6.4) in terms of $S$ :

$$
\begin{gathered}
\operatorname{det} \hat{\mathbf{R}}_{0}=\frac{1}{(N+L)^{J}} \operatorname{det}\left(\mathbf{S}+\sum_{n=1}^{N} \mathbf{x}[n] \mathbf{x}[n]^{H}\right), \\
\operatorname{det} \hat{\mathbf{R}}_{1}=\frac{1}{(N+L)^{J}} \operatorname{det}\left(\mathbf{S}+\sum_{n=1}^{N}(\mathbf{x}[n]-\mathbf{a} \hat{s}[n])(\mathbf{x}[n]-\mathbf{a} \hat{s}[n])^{H}\right),
\end{gathered}
$$

and taking into account the equality $\operatorname{det}\left(\mathbf{A}+\mathbf{x} \mathbf{x}^{H}\right)=\operatorname{det} \mathbf{A}\left(1+\mathbf{x}^{H} \mathbf{A}^{-1} \mathbf{x}\right)$ leads to

$$
l=\frac{1+\sum_{n=1}^{N} \mathbf{x}[n]^{H} \mathbf{S}^{-1} \mathbf{x}[n]}{1+\sum_{n=1}^{N}(\mathbf{x}[n]-\mathbf{a} \hat{s}[n])^{H} \mathbf{S}^{-1}(\mathbf{x}[n]-\mathbf{a} \hat{s}[n])} .
$$

Replacing $\hat{s}[n]$ with 6.5 , the test statistic is reduced to:

$$
\begin{gathered}
l=\frac{1+\sum_{n=1}^{N} \mathbf{x}[n]^{H} S^{-1} \mathbf{x}[n]}{1+\sum_{n=1}^{N}\left(\mathbf{x}[n]^{H} \mathbf{S}^{-1} \mathbf{x}[n]-\frac{\left|\mathbf{a}^{H} \mathbf{S}^{-1} \mathbf{x}[n]\right|^{2}}{\mathbf{a}^{H} \mathbf{S}^{-1} \mathbf{a}}\right)} \\
=1+\frac{\sum_{n=1}^{N} \frac{\left|\mathbf{a}^{H} \mathbf{S}^{-1} \mathbf{x}[n]\right|^{2}}{\mathbf{a}^{H} \mathbf{S}^{-1} \mathbf{a}}}{1+\sum_{n=1}^{N}\left(\mathbf{x}[n]{ }^{H} \mathbf{S}^{-1} \mathbf{x}[n]-\frac{\left|\mathbf{a}^{H} \mathbf{S}^{-1} \mathbf{x}[n]\right|^{2}}{\mathbf{a}^{H} \mathbf{S}^{-1} \mathbf{a}}\right)} .
\end{gathered}
$$

Using only the second term, the GLRT statistic becomes:

$$
t_{1}=\frac{\sum_{n=1}^{N} \frac{\left|\mathbf{a}^{H} \mathbf{S}^{-1} \mathbf{x}[n]\right|^{2}}{\mathbf{a}^{H} \mathbf{S}^{-1} \mathbf{a}}}{1+\sum_{n=1}^{N}\left(\mathbf{x}[n]{ }^{H} \mathbf{S}^{-1} \mathbf{x}[n]-\frac{\left|\mathbf{a}^{H} \mathbf{S}^{-1} \mathbf{x}[n]\right|^{2}}{\mathbf{a}^{H} \mathbf{S}^{-1} \mathbf{a}}\right)} .
$$

The detection decision between $H_{0}$ and $H_{1}$ is made by testing $t_{1}$ against a threshold.

Proposition: The test statistic $t_{1}$ in 6.12 can be manipulated into a form independent of the unknown parameters, except through the SNR, thus showing it is a Constant False Alarm Rate (CFAR) test.

\subsubsection{Detection Using a Random Source Signal Model}

This section presents another candidate detection rule where, rather than deterministic, we view the signal of interest, $s[n]$, as a white Gaussian noise process which is switched "on" 
and "off" to describe FRBs and other transients. In addition, the array feed is assumed to be calibrated, and thus the noise covariance matrix $\mathbf{R}_{\epsilon}$ is known or well estimated.

Consider $\mathbf{x}[n]=\left[x_{1}[n], \ldots, x_{J}[n]\right]^{T}$ the vector of sample observations by $J$ elements of a DAA. The superscript $T$ is used for a matrix "transposed" operation.

Two possible hypotheses are to be tested: hypothesis $H_{0}$, when the observed data are pure noise, and hypothesis $H_{1}$, when the signal is present. Defining $\mathbf{A}=\mathbf{a a}^{H}$, where as before $\mathbf{a}$ is the response of the array feed to $s[n]$, under these hypotheses $\mathbf{x}[n]$ is distributed as

$$
\begin{aligned}
H_{0}: \mathbf{x}[n]=\epsilon[n] & \sim C N\left(\mathbf{0}, \mathbf{R}_{\epsilon}\right), \quad n=1, \ldots N, \\
H_{1}: \mathbf{x}[n]=\mathbf{a} s[n]+\epsilon[n] & \sim C N\left(\mathbf{0}, \sigma_{s}^{2}[n] \mathbf{A}+\mathbf{R}_{\epsilon}\right), \quad n=1, \ldots N .
\end{aligned}
$$

The signal variance $\sigma_{s}^{2}[n]$ is a function of time. Since the stochastic description of the signal involves unknown parameters, we choose to work with a GLRT, where unknown parameters in the rule are replaced by the estimated values. Rules of this type are suboptimal, but are often asymptotically optimal (see [50,51,56] for more details). The GLRT is given by

$$
\frac{\max _{\left\{\sigma_{s}^{2}[1], \ldots, \sigma_{s}^{2}[n]\right\}} p_{1}(\mathbf{x})}{p_{0}(\mathbf{x})} .
$$

Consider the log-likelihood function $l_{1}$ in the numerator of (6.14) due to the model in $6.13):$

$$
l_{1}=-\sum_{n=1}^{N} \mathbf{x}[n]^{H}\left(\sigma_{s}^{2}[n] \mathbf{A}+\mathbf{R}_{\epsilon}\right)^{-1} \mathbf{x}[n]-\sum_{n=1}^{N} \log \operatorname{det}\left(\sigma_{s}^{2}[n] \mathbf{A}+\mathbf{R}_{\epsilon}\right) .
$$

We derive the optimal parameters in several steps. Applying Woodbury's identity to $\left(\sigma_{s}^{2}[n] \mathbf{A}+\mathbf{R}_{\epsilon}\right)^{-1}$

$$
\left(\sigma_{s}^{2}[n] \mathbf{A}+\mathbf{R}_{\epsilon}\right)^{-1}=\mathbf{R}_{\epsilon}^{-1}-\frac{\mathbf{R}_{\epsilon}^{-1} \sigma_{s}^{2}[n] \mathbf{a a}^{H} \mathbf{R}_{\epsilon}^{-1}}{1+\sigma_{s}^{2}[n] \mathbf{a}^{H} \mathbf{R}_{\epsilon}^{-1} \mathbf{a}}
$$

and substituting it in the expression under the first sum in 6.15 yields:

$$
-\sum_{n=1}^{N} \mathbf{x}[n]^{H} \mathbf{R}_{\epsilon}^{-1} \mathbf{x}[n]+\sum_{n=1}^{N} \frac{\sigma_{s}^{2}[n]}{1+\sigma_{s}^{2}[n] \mathbf{a}^{H} \mathbf{R}_{\epsilon}^{-1} \mathbf{a}} \mathbf{x}[n]^{H} \mathbf{R}_{\epsilon}^{-1} \mathbf{a a}^{H} \mathbf{R}_{\epsilon}^{-1} \mathbf{x}[n] .
$$

Differentiating (6.17) with respect to the unknown parameters $\sigma_{s}^{2}[n]$, leads to:

$$
\left(\frac{1}{1+\sigma_{s}^{2}[n] \mathbf{a}^{H} \mathbf{R}_{\epsilon}^{-1} \mathbf{a}}-\frac{\sigma_{s}^{2}[n] \mathbf{a}^{H} \mathbf{R}_{\epsilon}^{-1} \mathbf{a}}{\left(1+\sigma_{s}^{2}[n] \mathbf{a}^{H} \mathbf{R}_{\epsilon}^{-1} \mathbf{a}\right)^{2}}\right)\left|\mathbf{a}^{H} \mathbf{R}_{\epsilon}^{-1} \mathbf{x}[n]\right|^{2}=\frac{\left|\mathbf{a}^{H} \mathbf{R}_{\epsilon}^{-1} \mathbf{x}[n]\right|^{2}}{\left(1+\sigma_{s}^{2}[n] \mathbf{a}^{H} \mathbf{R}_{\epsilon}^{-1} \mathbf{a}\right)^{2}}
$$


Differentiating the second sum in 6.15 with respect to $\sigma_{s}^{2}[n]$, and applying (A.390) on page 1401 of VanTrees vol.4 [54,

$$
\frac{\partial}{\partial \sigma_{s}^{2}[n]} \log \operatorname{det}\left(\sigma_{s}^{2}[n] \mathbf{A}+\mathbf{R}_{\epsilon}\right)=\operatorname{Tr}\left[\left(\sigma_{s}^{2}[n] \mathbf{A}+\mathbf{R}_{\epsilon}\right)^{-1} \frac{\partial\left(\sigma_{s}^{2}[n] \mathbf{A}+\mathbf{R}_{\epsilon}\right)}{\partial \sigma_{s}^{2}[n]}\right]
$$

and using the Woodbury's identity from 6.16

$$
\begin{gathered}
\operatorname{Tr}\left[\mathbf{R}_{\epsilon}^{-1}-\frac{\sigma_{s}^{2}[n]}{1+\sigma_{s}^{2}[n] \mathbf{a}^{H} \mathbf{R}_{\epsilon}^{-1} \mathbf{a}} \mathbf{R}_{\epsilon}^{-1} \mathbf{a a}^{H} \mathbf{R}_{\epsilon}^{-1} \mathbf{a a}^{H}\right] \\
=\operatorname{Tr}\left[\mathbf{R}_{\epsilon}^{-1} \mathbf{a a}^{H}\right]-\operatorname{Tr}\left[\frac{\sigma_{s}^{2}[n]}{1+\sigma_{s}^{2}[n]\left(\mathbf{a}^{H} \mathbf{R}_{\epsilon}^{-1} \mathbf{a}\right)}\left(\mathbf{a}^{H} \mathbf{R}_{\epsilon}^{-1} \mathbf{a}\right)\left(\mathbf{R}_{\epsilon}^{-1} \mathbf{a a}^{H}\right)\right] \\
=\left(1-\frac{\sigma_{s}^{2}[n]\left(\mathbf{a}^{H} \mathbf{R}_{\epsilon}^{-1} \mathbf{a}\right)}{1+\sigma_{s}^{2}[n]\left(\mathbf{a}^{H} \mathbf{R}_{\epsilon}^{-1} \mathbf{a}\right)}\right) \operatorname{Tr}\left[\mathbf{R}_{\epsilon}^{-1} \mathbf{a a}^{H}\right] \\
=\frac{\operatorname{Tr}\left[\mathbf{R}_{\epsilon}^{-1} \mathbf{a a}^{H}\right]}{1+\sigma_{s}^{2}[n]\left(\mathbf{a}^{H} \mathbf{R}_{\epsilon}^{-1} \mathbf{a}\right)} .
\end{gathered}
$$

Combining (6.18) and 6.20), the maximum likelihood estimates of the unknown parameters $\sigma_{s}^{2}[n], n=1, \ldots, N$ are

$$
\hat{\sigma}_{s}^{2}[n]=\frac{\mathbf{x}[n]^{H} \mathbf{R}_{\epsilon}^{-1} \mathbf{a a}^{H} \mathbf{R}_{\epsilon}^{-1} \mathbf{x}[n]-T r\left[\mathbf{R}_{\epsilon}^{-1} \mathbf{a a}^{H}\right]}{\operatorname{Tr}\left[\mathbf{R}_{\epsilon}^{-1} \mathbf{a} \mathbf{a}^{H}\right]\left(\mathbf{a}^{H} \mathbf{R}_{\epsilon}^{-1} \mathbf{a}\right)},
$$

where $\operatorname{Tr}(\cdot)$ denotes the trace of a matrix. Taking into account the complex Gaussian models for the signal and noise, the logarithm of (6.14) with the true variances replaced by their estimates can be reduced to:

$$
t_{2}=\sum_{n=1}^{N} \frac{\left|S_{1}(\mathbf{x}[n])\right|^{2}-S_{2}}{S_{3}}-\sum_{n=1}^{N} \log \operatorname{det}\left(\frac{\left|S_{1}(\mathbf{x}[n])\right|^{2}-S_{2}}{S_{2} S_{3}} \mathbf{R}_{\epsilon}^{-1} \mathbf{a a}^{H}+\mathbf{I}\right)
$$

where $S_{1}(\mathbf{x}[n])=\mathbf{a}^{H} \mathbf{R}_{\epsilon}^{-1} \mathbf{x}[n], S_{2}=\operatorname{Tr}\left[\mathbf{R}_{\epsilon}^{-1} \mathbf{a a}^{H}\right], S_{3}=\mathbf{a}^{H} \mathbf{R}_{\epsilon}^{-1} \mathbf{a}$, and $\mathbf{I}$ is an $J \times J$ identity matrix. The detection decision between $H_{0}$ and $H_{1}$ is made by testing $t_{2}$ against a threshold. Comment: Note that $t_{2}$ is a nonlinear transformation of the sufficient statistic $S_{1}(\mathbf{x}[n])$, which yields an optimized decision with the GLRT. It is interesting to note that $S_{1}(\mathbf{x}[n])$ can be recognized as the maximum SNR beamformer solution which has been widely used by astronomical DAA practitioners [47,48, 53]. 


\subsection{Numerical Evaluation}

In the absence of publicly available test data from a radio astronomical phased array and a limited access to simulated data, the performance of the detection rules described in Section 6.3 is demonstrated on time series of a single pixel telescope. We used two time series containing signals of two different millisecond pulsars. The time series were acquired as a part of calibration observations taken by the $820 \mathrm{MHz}$ receiver [57] and the GUPPI backend [58] on the Green Bank Telescope. The two linear polarizations of the receiver are sampled by 8-bit A/Ds at $400 \mathrm{MHz}$, to achieve Nyquist sampling of a $200 \mathrm{MHz}$ passband. These individual time samples are then passed through a polyphase filter bank and a fast Fourier transform. Next, the two polarizations are combined to form Stokes parameters (a set of values that describe the polarization state of electromagnetic radiation). Finally, these spectra are accumulated for the hardware time of $82 \mu s$. The two millisecond pulsars are $B 1937+21$ and $J 1125+78$, with periods of 1.56 and $4.20 \mathrm{~ms}$ respectively. We emphasise that we are not searching for periodic signals here, and that knowledge of the pulse period is not required for the decision rules. Rather, this information is used to generate ground truth locations of signal pulses in the time series.

The series $B 1937+21$ is known as a strong millisecond pulsar, while $J 1125+78$ is a medium strength pulsar. A short excerpt from B1937 +21 is shown in Fig. 6.1. Red circles show two adjacent signal pulses. Both time series are de-dispersed prior to applying any other signal processing. Both time series have a large DC component due to the "sky" background noise.

For a single horn receiver, the two detection rules introduced in Section 6.3 are reduced to the following basic test statistics. The detection rule $t_{1}$ is reduced to a rule similar to the one used by radio astronomers at West Virginia University [59]. Denote the rule as $g_{1}$ :

$$
g_{1}=\sum_{n=1}^{N}|x[n]|^{2} \begin{cases}\geq \gamma & \text { pulse is detected } \\ <\gamma & \text { no pulse is detected }\end{cases}
$$

The detection rule $t_{2}$ is reduced to the rule denoted as $g_{2}$ :

$$
g_{2}=\sum_{n=1}^{N}\left(\frac{|x[n]|^{2}}{\sigma_{\epsilon}^{2}}-\log \frac{|x[n]|^{2}}{\sigma_{\epsilon}^{2}}-1\right) \begin{cases}\geq \gamma & \text { pulse is detected } \\ <\gamma & \text { no pulse is detected }\end{cases}
$$




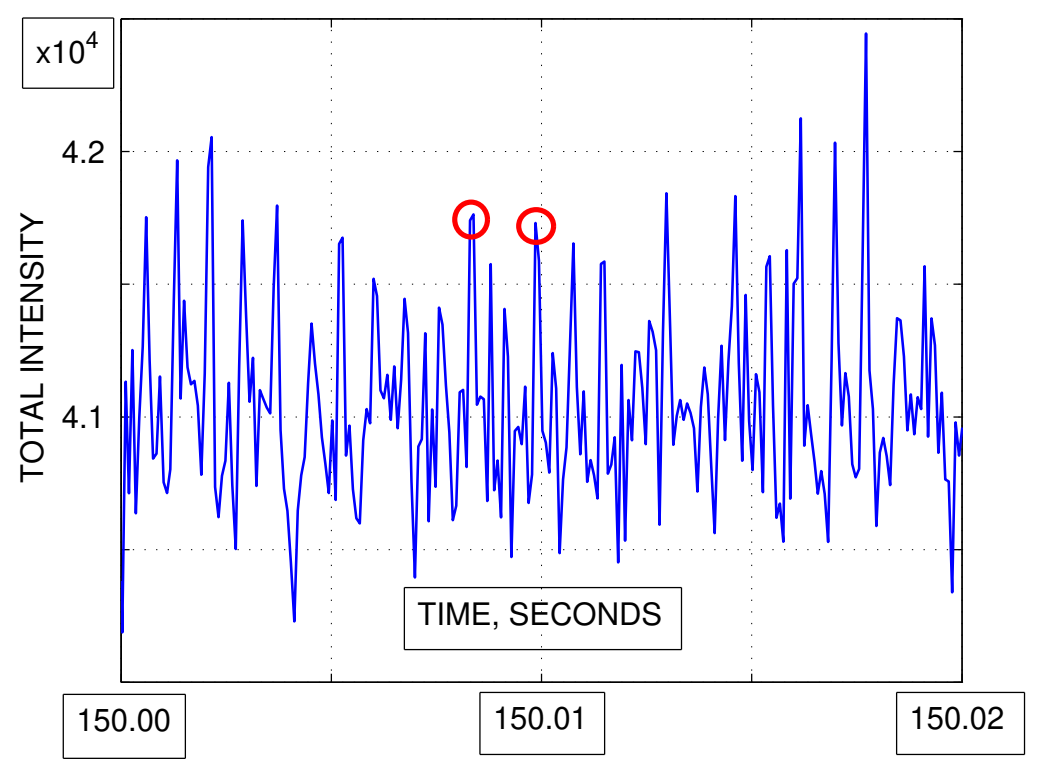

Figure 6.1: Shown is a 20 millisecond interval of the $B 1937+21$ time series. Two red circles mark two adjacent pulses of the signal of interest. Note that some of the noise pulses have much larger amplitude than the signal pulses.

Recall that both rules are designed assuming that the data contain an additive complex Gaussian noise and a signal modeled as a deterministic signal for rule $g_{1}$ and as a complex Gaussian signal with its variance as a function of time for rule $g_{2}$.

In addition, we introduce a third rule making use of the known fact that the received data have been converted into energy samples. This rule is based on the assumption that the signal and noise forming raw data $x[n]$ are independent zero mean white complex Gaussian processes with different second order statistics. Then a new random variable $y[n]=|x[n]|^{2}$ is chi-square distributed under both hypotheses. Denoting the new rule as $g_{3}$ and assuming that the signal $s[n]$ has unknown variance, the GLRT rule for this case is reduced to:

$$
g_{3}=\max \left\{\frac{\sum_{n=1}^{N} y[n]}{N \sigma_{\epsilon}^{2}}, 1\right\}-\log \max \left\{\frac{\sum_{n=1}^{N} y[n]}{N \sigma_{\epsilon}^{2}}, 1\right\} \begin{cases}\geq \gamma & \text { pulse is detected } \\ <\gamma & \text { no pulse is detected }\end{cases}
$$

To quantify the detection performance of the three detection rules, the time series were partitioned into nonoverlapping intervals of length $N$. The three rules are applied to each interval, and the value of the test statistics is compared to a threshold. Since the ground truth location of pulses has been pre-calculated, we are able to differentiate between "true 

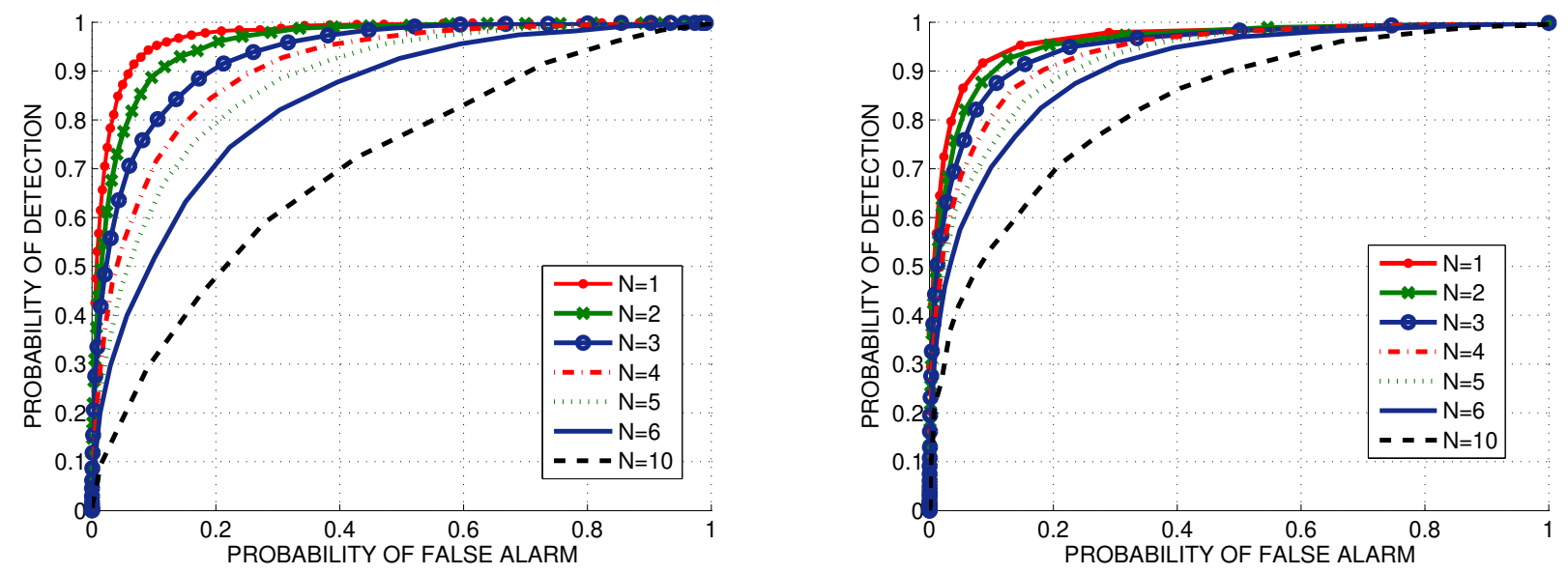

Figure 6.2: Performance of the rules $g_{1}$ (left panel) and $g_{2}$ (right panel) applied to the $B 1937+21$ time series and parameterized by the number of time samples used to implement the rules.

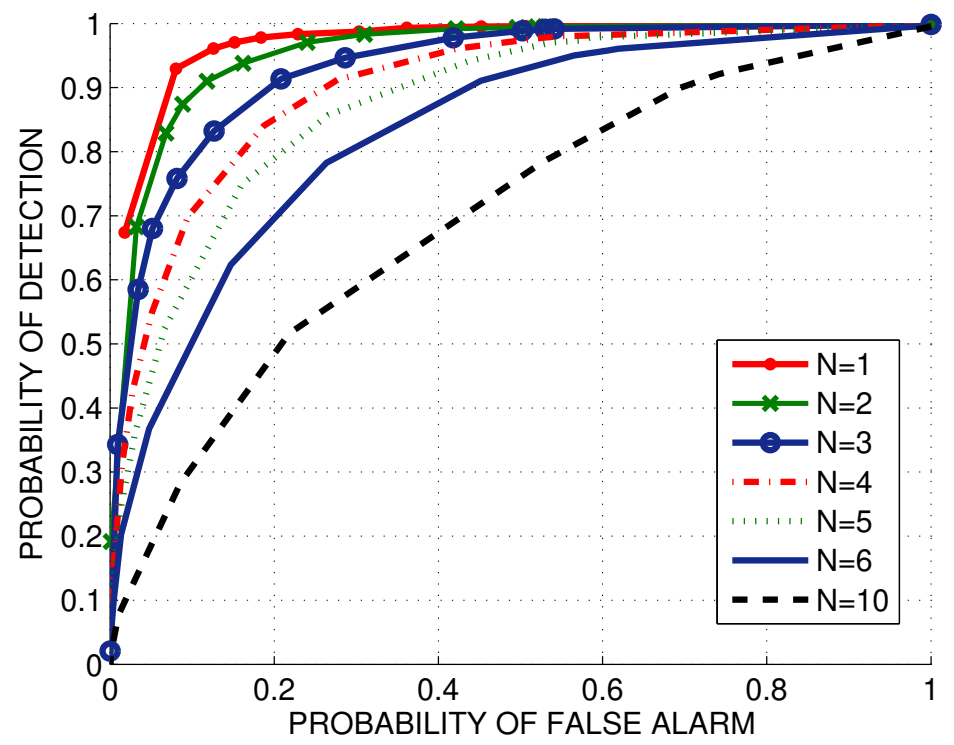

Figure 6.3: Performance of the rules $g_{3}$ applied to the $B 1937+21$ time series and parameterized by the number of time samples used to implement the rules. 
detections" and "false alarms." Counting the number of correct detections and false alarms and varying the value of the threshold $\gamma$ allows us to display these statistics as a Receiver Operating Characteristic curves (ROCs) known in classical detection and estimation as a complete characterization of a detection rule. In addition to varying the value of $\gamma$ for each rule, we also vary the value of $N$. Fig. 6.2 and 6.3 display the ROC curves parameterized by seven differen values of $N$. Note that increasing $N$ further would lead to inability to evaluate "false alarm," since the difference between two pulses in the series $B 1937+21$ is represented by only 19 time samples.

It is interesting to observe that while the rules $g_{1}$ and $g_{3}$ perform very well when $N$ is set to one, the rule $g_{2}$ outperforms the other two rules for the values of $N$ above one. Furthermore, it appears that the rule $g_{2}$ is quite robust to the choice of $N$. The six lowest- $N$ ROC curves are clustered closely together.

Fig. 6.4 show the performance for the time series $J 1125+78$. As observed, at a lower value of the SNR, the detection performance degrades substantially. Furthermore, there is nearly no distinction between the ROC curves for the rules $g_{1}, g_{2}$ and $g_{3}$. Because of this, we do not display the ROCs of the rule $g_{3}$. As it appears from the results, all three rules rely heavily on the original data disregarding the logarithm term used in the last two rules. We have attempted to apply a smoothing transformation to the data with the goal to reduce the noise level. This resulted in overall performance improvement. However, it did not change the ranking of the rules. Once again, they demonstrated nearly identical performance.

\subsection{Summary}

This work has developed two detection rules for receiving an unknown astronomical signal immersed in large additive noise using data observed by a phased array. The two rules relied on two sets of different assumptions about the signal and noise. To develop the first rule, the signal was modeled as an unknown deterministic function and the noise was viewed as a complex Gaussian process with unknown correlation function of the elements in the array. The correction function was estimated involving secondary data that were selected to be "signal free." Since the models included unknown parameters, the first rule was built as 

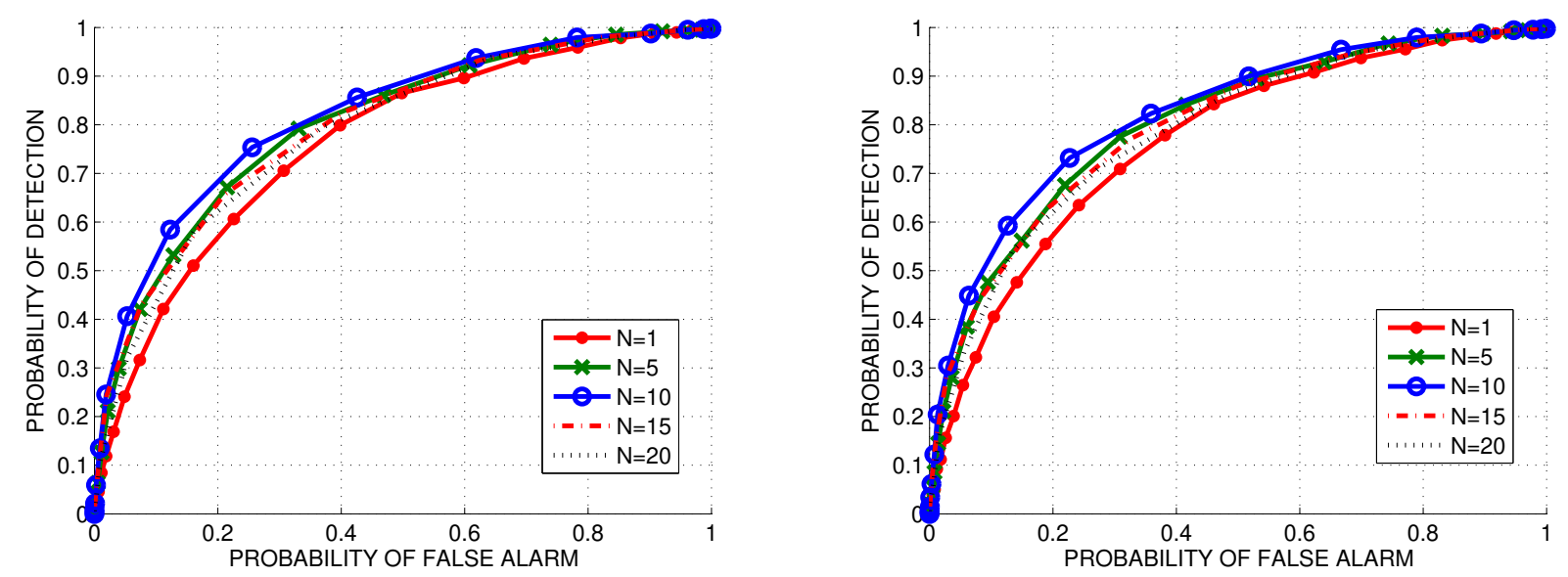

Figure 6.4: Performance of the rules $g_{1}$ (left panel) and $g_{2}$ (right panel) applied to the $J 1125+78$ time series and parameterized by the number of time samples used to implement the rules.

a GLRT rule. The second rule made more realistic assumptions about the nature of an astronomical signal of interest and the additive noise in the data. They were modeled as independent complex Gaussian processes in time. Under this set up, the correlation function of noise samples in the element of a phased array is assumed to be known and the variance of the signal as a function of time is unknown. The second rule was developed as a new GLRT rule and involved many different sufficient statistics compared to the first rule.

In the absence of real test data, the numerical analysis involved real test data from a single pixel telescope. Time series of two millisecond pulsars (one of high SNR and the other known as a medium SNR) were timed and partitioned into non-overlapping intervals of a very short duration. Timing of these pulsars provided a ground truth of signal pulse locations in the time series. By applying the two developed rules to each time interval and comparing the value of the rule to a threshold, we were able to evaluate the probability of correct detection of signal pulses and the probability of false alarm for each of the two rules. By varying the threshold and the width of the interval, we displayed results of the performance evaluation as a family of ROC curves. It is interesting and pleasing to note that in the case of the strong millisecond pulsar the second rule outperformed the first rule, which can be explained by the application of a wrong signal model in developing the first 
rule.

In developing the two rules, we assumed that the observed data were preprocessed. To be more specific, we assumed that the majority of the radio frequency interference would be detected and removed, and the raw time series would be de-dispersed using a conventional approach. While we are aware of the inefficiency of the conventional algorithm when applied to array data, a new algorithm that takes into account the geometry of an array has not yet been developed. 


\section{Chapter 7}

\section{Proposed Future Work}

\subsection{Stochastic description of raw voltages}

We propose to develop a new stochastic model for the description of a dispersed pulse in raw telescope data, then develop an optimal rule to detect the signal. From Information Theory perspective [60], raw data contain more information compared to processed data.

To begin, we note that observed data on the output of a telescope are raw voltages collected as a function of time. A transient signal $s(t)$ observed by a telescope can be modeled as a dispersed deterministic Gaussian bell function (envelope of the signal) modulated by a white Gaussian noise process.

Let $g(t)$ be the shaping function of the transient pulse defined as

$$
g(t)=\exp \left(-t^{2} /\left(2 \sigma^{2}\right)\right) / \sqrt{2 \pi \sigma^{2}} .
$$

The transfer function of the Interstellar Medium (ISM) is provided in Lorimer and Kramer [52]:

$$
H(f)=\exp \left(j \frac{2 \pi \times 4.15 \times 10^{3}}{f f_{0}^{2}} \times D M \times\left(f-f_{0}\right)^{2}\right),
$$

where $f_{0}$ is the center frequency, $f$ is a frequency withing the bandwidth $B W$, that is, $f \in\left(f_{0}-B W / 2, f_{0}+B W / 2\right)$ measured in $\mathrm{MHz}$, and $D M$ is the dispersion measure in $\mathrm{pc} / \mathrm{cm}^{3}$. Given the notation above, the Fourier transform of the dispersed signal $s(t)$ observed on the interval $\left(t_{i}, t_{f}\right)$ is modeled as

$$
S(f)=H(f) \int_{t_{i}}^{t_{f}} n(t) g(t) \exp (-j 2 \pi f t) d t,
$$


where $n(t)$ is a white Gaussian random signal with power spectral density $P_{0} / 2$ and the parameter $\sigma^{2}$ determines the width of the transient pulse

Since both transient signal and noise are Gaussian signals, their Fourier transforms are also Gaussian. Furthermore, due to the independence of signal and noise at the receiver end, their Fourier transforms are also independent signals. The received signal on the output of a telescope including a transient signal and the receiver noise is mathematically described as follows:

$$
X(f)=S(f)+E(f)
$$

where $S(f)$ is Gaussian distributed with mean zero and covariance function of $\mathbf{R}_{S}\left(f_{1}, f_{2}\right)$, the noise $E(f)$ is also a Gaussian signal with zero mean and covariance function $\mathbf{R}_{E}$, and $f, f_{1}, f_{2} \in\left(f_{0}-B W / 2, f_{0}+B W / 2\right)$.

\subsection{Detection rule}

Based on the proposed models for the transient signal and noise, the problem of detecting a transient signal can be formulated as a binary hypothesis testing problem. The two possible hypotheses to be tested are: hypothesis $H_{0}$, when the observed data are pure noise, and hypothesis $H_{1}$, when the transient signal is present. Then under $H_{0}$ and $H_{1}$ hypotheses $\mathbf{X}(f)$ is distributed as: Let $\mathbf{X}$ be a vector samples of data $X(f)$ at $n$ equally spaced discrete frequencies, then under $H_{0}$ and $H_{1}$ hypotheses $\mathbf{X}$ is distributed as:

$$
\begin{aligned}
& H_{0}: \quad \mathbf{X} \sim C N\left(\mathbf{0}, \mathbf{R}_{E}\right) \\
& H_{1}: \quad \mathbf{X} \sim C N\left(\mathbf{0}, \mathbf{R}_{S}+\mathbf{R}_{E}\right),
\end{aligned}
$$

where the covariance matrix $\mathbf{R}_{S}$ is unknown and thus has to be estimated from the data. The hypothesis testing problem leading to a Generalized Likelihood Ratio (GLRT). Denote by $\hat{\mathbf{R}}_{S}$ an estimate of the unknown matrix $\mathbf{R}_{S}$. Then GLRT decides that the transient signal is present if

$$
-\mathbf{X}^{H}\left(\left(\hat{\mathbf{R}}_{S}+\mathbf{R}_{E}\right)^{-1}-\mathbf{R}_{E}^{-1}\right) \mathbf{X}-\log \operatorname{det}\left(\left(\hat{\mathbf{R}}_{S}+\mathbf{R}_{E}\right) \mathbf{R}_{E}^{-1}\right)>\tau,
$$

where $\tau$ is a design parameter, a threshold. If the inequality in 7.5 does not hold, then GLRT decides that data do not contain a transient signal. 
A possible approach to estimating $R_{S}\left(f_{1}, f_{2}\right)$ from data is to represent the function as a linear combination of a set of some known basis functions such as two-dimensional spline functions, radial basis functions, wavelets, and other possible sets of functions. Given the number of basis functions and their placement in $\left(f_{1}, f_{2}\right)$ plane, the problem of estimating $R_{S}\left(f_{1}, f_{2}\right)$ is reduced to the estimation of a finite number of weights, one per each basis function. The number of basis functions used to estimate $R_{S}\left(f_{1}, f_{2}\right)$ depends on the amount of available data. It can be selected to optimize a bias-variance decomposition, Akaike Information Criterion, Bayesian Information Criterion, Minimum Description Length, Maximum entropy principle or it can be stated as a maximum likelihood estimation problem under the constraint imposed by Grenander's method of sieves. 


\section{Appendix A}

\section{Related Publications}

- M. Alkhweldi and N. A. Schmid, "Application of the Radon transform for search of candidate pulses in radio astronomy," Journal of Astronomical Telescopes, Instruments, and Systems (JATIS), Jan. 2020.

- M. Alkhweldi and N. A. Schmid, "Spatial Fourier Transform for Detection and Analysis of Periodic Astrophysical Pulses," IEEE Global Conference on Signal and Information Processing (GlobalSIP), Nov. 2018.

- M. Alkhweldi, N. A. Schmid, and R. Prestage, "A blind transform based approach for the detection of isolated astrophysical pulses, "IEEE International Conference on Acoustics, Speech and Signal Processing (ICASSP), Mar. 2017.

- M. Alkhweldi, R. Prestage, R. Lynch, and N. A. Schmid, "On the blind detection of FRBs through spatial fourier transforms, " United States National Committee of URSI National Radio Science Meeting (USNC-URSI NRSM), 2016.

- N. Schmid, R. Prestage, and M. Alkhweldi, "Inferential statistics for transient signal detection in radio astronomy phased arrays," in Proc. SPIE Automatic Target Recognition XXV, 2015. 


\section{References}

[1] G. Doran, "Characterizing interference in radio astronomy observations through active and unsupervised learning," JPL Publication 13-12, 2013.

[2] A. Krizhevsky, I. Sutskever, and G. E. Hinton, "Imagenet classification with deep convolutional neural networks," in Advances in Neural Information Processing Systems (F. Pereira, C. J. C. Burges, L. Bottou, and K. Q. Weinberger, eds.), vol. 25, Curran Associates, Inc., 2012.

[3] D. R. Lorimer, M. Bailes, M. A. McLaughlin, D. J. Narkevic, and F. Crawford, "A bright millisecond radio burst of extragalactic origin," Science, vol. 318, pp. 777-780, Nov. 2007.

[4] D. Thornton, B. Stappers, M. Bailes, B. Barsdell, S. Bates, N. D. R. Bhat, M. Burgay, S. Burke-Spolaor, D. J. Champion, P. Coster, N. D'Amico, A. Jameson, S. Johnston, M. Keith, M. Kramer, L. Levin, S. Milia, C. Ng, A. Possenti, and W. van Straten, "A population of fast radio bursts at cosmological distances," Science, vol. 341, pp. 53-56, July 2013.

[5] E. Petroff, E. Barr, A. Jameson, E. Keane, M. Bailes, M. Kramer, V. Morello, D. Tabbara, and W. van Straten, "Frbcat: The fast radio burst catalogue," arXiv preprint arXiv:1601.0354 \%v1, 2016.

[6] H. Falcke and L. Rezzolla, "Fast radio bursts: the last sign of supramassive neutron stars," Astronomy \& Astrophysics, vol. 562, p. 137, 2014.

[7] T. Totani, "Cosmological Fast Radio Bursts from Binary Neutron Star Mergers," PASJ, vol. 65, p. 12, 2014.

[8] M. J. Rees, "A better way of searching for black-hole explosions," Nature, vol. 266, p. 333, Mar. 1977.

[9] J. B. Burnell, "Little green men, white dwarfs or pulsars?," Annals of the New York Academy of Science, vol. 302, pp. 685-689, 1977.

[10] D. Lorimer and M. Kramer, Handbook of pulsar astronomy. New York: Cambridge University Press, 2012.

[11] E. Keane, M. Kramer, A. Lyne, B. Stappers, and M. McLaughlin, "Rotating radio transients: new discoveries, timing solutions and musings," Monthly Notices of the Royal Astronomical Society, vol. 415, no. 4, pp. 3065-3080, 2011. 
[12] RRATalog, "The rratalog," http://astro.phys.wvu.edu/rratalog/, 2016.

[13] M. Keith, R. Eatough, A. Lyne, M. Kramer, A. Possenti, F. Camilo, and R. Manchester, "Discovery of 28 pulsars using new techniques for sorting pulsar candidates," Monthly Notices of the Royal Astronomical Society, vol. 395, no. 2, pp. 837-846, 2009.

[14] X.-D. Li, "On the nature of part-time radio pulsars," The Astrophysical Journal Letters, vol. 646, no. 2, p. L139, 2006.

[15] Q. Luo and D. Melrose, "Pulsar radiation belts and transient radio emission," Monthly Notices of the Royal Astronomical Society, vol. 378, no. 4, pp. 1481-1490, 2007.

[16] J. M. Cordes and R. Shannon, "Rocking the lighthouse: circumpulsar asteroids and radio intermittency," The Astrophysical Journal, vol. 682, no. 2, p. 1152, 2008.

[17] P. Weltevrede, B. Stappers, J. Rankin, and G. Wright, "Is pulsar b0656+ 14 a very nearby rotating radio transient?," The Astrophysical Journal Letters, vol. 645, no. 2, p. L149, 2006.

[18] M. A. McLaughlin, A. Lyne, D. Lorimer, M. Kramer, A. Faulkner, R. Manchester, J. Cordes, F. Camilo, A. Possenti, I. Stairs, et al., "Transient radio bursts from rotating neutron stars," Nature, vol. 439, no. 7078, pp. 817-820, 2006.

[19] R. M. Prestage, K. T. Constantikes, T. R. Hunter, L. J. King, R. J. Lacasse, F. J. Lockman, and R. D. Norrod, "The Green Bank Telescope," IEEE Proceedings, vol. 97, pp. 1382-1390, Aug. 2009.

[20] J. Cordes and M. A. McLaughlin, "Searches for fast radio transients," The Astrophysical Journal, vol. 596, no. 2, p. 1142, 2003.

[21] S. M. Ransom, New search techniques for binary pulsars. PhD thesis, Harvard University Cambridge, Massachusetts, 2001.

[22] B. R. Barsdell, M. Bailes, D. G. Barnes, and C. J. Fluke, "Accelerating incoherent dedispersion," Monthly Notices of the Royal Astronomical Society, vol. 422, p. 379-392, 2012.

[23] N. Clarke, J. P. Macquart, and C. Trott, "Performance of a novel fast transient detection system," The Astrophysical Journal Supplement Series, vol. 205, p. 4, Feb. 2013.

[24] B. Zackay and E. O. Ofek, "An accurate and efficient algorithm for detection of radio bursts with an unknown dispersion measure, for single dish telescopes and interferometers," The Astrophysical Journal, 2014.

[25] L. G. Spitler, J. M. Cordes, J. W. T. Hessels, D. R. Lorimer, M. A. McLaughlin, S. Chatterjee, F. Crawford, J. S. Deneva, V. M. Kaspi, R. S. Wharton, B. Allen, S. Bogdanov, A. Brazier, F. Camilo, P. C. C. Freire, F. A. Jenet, K.-A. C., B. Knispel, P. Lazarus, K. J. Lee, J. van Leeuwen, R. Lynch, S. M. Ransom, P. Scholz, X. Siemens, I. H. Stairs, K. Stovall, J. K. Swiggum, A. Venkataraman, W. W. Zhu, C. Aulbert, and H. Fehrmann, 
"Fast radio burst discovered in the arecibo pulsar alfa survey," The Astrophysical Journal, vol. 790, p. 101, Jul. 2014.

[26] K. Masui, H.-H. Lin, J. Sievers, C. J. Anderson, T.-C. Chang, X. Chen, A. Ganguly, M. Jarvis, C.-Y. Kuo, Y.-C. Li, et al., "Dense magnetized plasma associated with a fast radio burst," Nature, vol. 528, no. 7583, pp. 523-525, 2015.

[27] D. Ait-Allal, R. Weber, C. Dumez-Viou, I. Cognard, and G. Theureau, "Blind detection of giant pulses: Gpu implementation," Comptes Rendus Physique, vol. 13, no. 1, pp. 80$87,2012$.

[28] B. Zackay and E. O. Ofek, "An accurate and efficient algorithm for detection of radio bursts with an unknown dispersion measure, for single dish telescopes and interferometers," The Astrophysical Journal, vol. 835, no. 11, p. 13, 2017.

[29] M. Alkhweldi, N. Schmid, and R. Prestage, "A blind transform based approach for the detection of isolated astrophysical pulses," in Proc. of the 2017 IEEE International Conference on Acoustics, Speech and Signal Processing (ICASSP), IEEE, 2017.

[30] N. A. Schmid and R. M. Prestage, "A Blind Transform Based Approach for the Detection of Isolated Astrophysical Pulses," Monthly Notices of the Royal Astronomical Society, vol. 477, pp. 4052-4062, April 2018.

[31] P. V. C. Hough, "Methods and means for recognizing complex methods," US patent 3,069,654, 1962.

[32] L. Connor and J. Van Leeuwen, "Applying deep learning to fast radio burst classification," ArXiv Astrophysics e-prints, arXiv:1803.03084v1, 2018.

[33] Y. G. Zhang, V. Gajjar, G. Foster, A. Siemion, J. Cordes, C. Law, and Y. Wang, "Fast radio burst 121102 pulse detection and periodicity: A machine learning approach," ArXiv Astrophysics e-prints, arXiv:1809.03043v1, 2018.

[34] D. Agarwal, K. Aggarwal, S. Burke-Spolaor, D. Lorimer, and N. Garver-Daniels, "Towards deeper neural networks for fast radio burst detection," Feb. 2019.

[35] A. T. L. B. Zhang HY, Zhao Z and N. Chen X, "Pulsar candidate recognition with deep learning," Computers and Electrical Engineering, vol. 73, pp. 1-8, Jan. 2019.

[36] M. Alkhweldi, N. A. Schmid, and R. M. Prestage, "A blind transform based approach for the detection of isolated astrophysical pulses," in Procedeeings of the 2017th IEEE International Conference on Acoustics, Speech and Signal Processing, 2017.

[37] S. Deans, The radon transform and some of its applications. New York: John Wiley and Sons, 1983.

[38] H. Van Trees, Detection, Estimation, and Modulation Theory, Part I. John Wiley and Sons, 2001. 
[39] T.-C. Chang, U.-L. Pen, K. Bandura, and J. B. Peterson, "An intensity map of hydrogen 21-cm emission at redshift z [thinsp][ap][thinsp] 0.8," Nature, vol. 466, no. 7305, pp. 463$465,2010$.

[40] N. A. Schmid and R. M. Prestage, "Blind detection of isolated astrophysical pulses in the spatial fourier transform domain," Monthly Notices of the Royal Astronomical Society, vol. 477, no. 3, pp. 4052-4062, 2018.

[41] A. T. R. N. Manchester, G. B. Hobbs and M. Hobbs, "The ATNF Pulsar Catalogue," Astronomical Journal, vol. 129, pp. 1993-2006, 2005.

http://www .atnf.csiro.au/research/pulsar/psrcat.

[42] R. J. Mailloux, Phased Array Antenna Handbook. Boston, MA: Artech House, 2005.

[43] W. W. Camp, J. T. Mayhan, and R. M. O’Donnell, "Wideband radar for ballistic missile defense and range-doppler imaging of satellites," Lincoln Laboratory Journal, vol. 12, pp. 267-280, July 2000.

[44] E. Brookner, "Phased array radars," Scientific American, vol. 252, pp. 94-102, Jan. 1985.

[45] J. Fisher, "Astro2010 technology development white paper: Phased array feeds." Available at https://science.nrao.edu/science/, 2010.

[46] W. A. Van Cappellen, L. Bakker, and T. A. Oosterloo, "Experimental results of the apertif phased array feed," in General Assembly and Scientific Symposium, 2011 XXXth URSI, pp. 1-4, IEEE, 2011.

[47] B. Jeffs, K. Warnick, J. Landon, J. Waldron, J. F. D. Jones, and R. Norrod, "Signal processing for phased array feeds in radio astronomical telescopes," IEEE Journal of Selected Topics in Signal Processing, vol. 2, pp. 635-646, Oct. 2008.

[48] M. Elmer, B. Jeffs, K. Warnick, J. Fisher, and R. Norrod, "Beamformer design methods for radio astronomical phased array feeds," IEEE Transactions on Antennas and Propagation, vol. 60, pp. 903-914, Feb. 2012.

[49] M. A. McLaughlin, A. G. Lyne, D. R. Lorimer, M. Kramer, A. J. Faulkner, R. N. Manchester, J. M. Cordes, F. Camilo, A. Possenti, I. H. Stairs, G. Hobbs, N. D'Amico, M. Burgay, and J. T. O'Brien, "Transient radio bursts from rotating neutron stars," Nature, vol. 439, pp. 817-820, Feb. 2006.

[50] H. Van Trees, Detection, Estimation, and Modulation Theory, Part I. John Wiley and Sons, 2001.

[51] V. Poor, An Introduction to Signal Detection and Estimation. New York: Springer, 2010.

[52] D. Lorimer and M. Kramer, Handbook of Pulsar Astronomy. Cambridge: Cambridge University Press, 2005. 
[53] M. Elmer, B. Jeffs, and K. Warnick, "Long-term calibration stability of a radio astronomical phased array feed," Astronomical Journal, vol. AJ 145, Jan. 2013.

[54] H. Van Trees, Detection, Estimation, and Modulation Theory, Part IV, Optimum Array Processing. John Wiley and Sons, 2002.

[55] R. J. Muirhead, Aspects of Multivariate Statistical Theory. New York: Wiley Interscience, 2005.

[56] A. Dembo and O. Zeitouni, Large Deviations Techniques and Applications. New York: Springer, 2009.

[57] "The proposer's guide for the green bank telescope." Available at https://science.nrao.edu/facilities/gbt/proposing/GBTpg.pdf/, 2015.

[58] R. DuPlain, S. Ransom, P. Demorest, P. Brandt, J. Ford, and A. Shelton, "Launching guppi: the green bank ultimate pulsar processing instrument," Proceedings of the SPIE Conf. on Advanced Software and Control for Astronomy II, vol. 7019, p. 70191D, Aug. 2008 .

[59] M. A. McLaughlin. private communication, 2015.

[60] T. M. Cover and J. A. Thomas, Elements of Information Theory (Wiley Series in Telecommunications and Signal Processing). USA: Wiley-Interscience, 2006. 